\title{
Effectiveness of "counseling as a related service" in Hawaii's public schools as measured by the Behavior Assessment System for Children, Second Edition (BASC-2)
}

Gregory A. Lobb

West Virginia University

Follow this and additional works at: https://researchrepository.wvu.edu/etd

\author{
Recommended Citation \\ Lobb, Gregory A., "Effectiveness of "counseling as a related service" in Hawaii's public schools as \\ measured by the Behavior Assessment System for Children, Second Edition (BASC-2)" (2006). Graduate \\ Theses, Dissertations, and Problem Reports. 2442. \\ https://researchrepository.wvu.edu/etd/2442
}

This Dissertation is protected by copyright and/or related rights. It has been brought to you by the The Research Repository @ WVU with permission from the rights-holder(s). You are free to use this Dissertation in any way that is permitted by the copyright and related rights legislation that applies to your use. For other uses you must obtain permission from the rights-holder(s) directly, unless additional rights are indicated by a Creative Commons license in the record and/ or on the work itself. This Dissertation has been accepted for inclusion in WVU Graduate Theses, Dissertations, and Problem Reports collection by an authorized administrator of The Research Repository @ WVU. For more information, please contact researchrepository@mail.wvu.edu. 
Effectiveness of "Counseling as a Related Service" in Hawaii's Public Schools as Measured by the Behavior Assessment System for Children, Second Edition (BASC-2)

\author{
Gregory A. Lobb
}

Dissertation submitted to the College of Human Resources and Education at West Virginia University in partial fulfillment of the requirements for the degree of

Doctor of Philosophy

in

Counseling Psychology

\author{
Roy Tunick, Ed.D., Chair Person \\ Margaret Glenn, Ed.D. \\ James DeLo, Ed.D. \\ Richard Walls, Ph.D. \\ Nancy Kennedy, Psy.D.
}

\title{
Morgantown, West Virginia 2006
}

Keywords: Adaptive Skills, School Problems, Internalizing Problems, Externalizing Problems, "counseling as a related service", counseling providers, school-based mental health services, BASC-2, Felix Consent Decree, Comprehensive Student Support System, Level 4 Services, Level 5 Services

Copyright 2006 Gregory A. Lobb 


\begin{abstract}
Effectiveness of "Counseling as a Related Service" in Hawaii's Public Schools as Measured by the Behavior Assessment System for Children, Second Edition (BASC-2)
\end{abstract}

Gregory A. Lobb

This study examined the effectiveness of "counseling as a related service" in Hawaii's public schools by examining the improvement on a teacher rating scale and also examining counseling minutes received by these same students. This study utilized 346 students receiving Level 4 and Level 5 services who were first refereed for "counseling as a related service" during the first quarter of 2055. Improvement is measured using the Behavioral Assessment System for Children, Second Edition (BASC-2) (Reynolds \& Kamphaus, 2004). Findings revealed that counseling interventions indicated statistically significant change in T Scores on the BASC-2 from Pre assessment to Post assessment. Multivariate Analyses of Variance were performed on composite scales and subscales of the BASC-2 at Pre assessment and Post assessment to address the main function of this paper. Follow-up ANOVAs were performed where statistically indicated. This study also examined the impact of minutes of specific counseling interventions (individual counseling, group counseling, and family education) on change in T Scores from Pre assessment to Post assessment on the BASC-2 TRS. The specific counseling minutes did not indicate a statistically significant change as was hypothesized. Regression analyses and correlation analyses were performed with no statistically significant results. T Scores were grouped into categories of significance and examined using Chi-Square analyses. Results indicate statistically significant results. Also discussed are clinical applications and suggestions for future research. 


\section{DEDICATION}

I would like to dedicate this work to my wife, Jill and my children, Alyssa, Anthony, and Jason, who with their love and support have made this, like everything else, possible. 


\section{ACKNOWLEDGMENTS}

I would first like to thank my committee chair, Dr. Roy Tunick for his persistent support and encouragement throughout this project. Without this I would have not been successful at completing this daunting task. Dr. Tunick has been a true advisor and mentor during my tenure at West Virginia University. I would also like to thank my committee members: Margaret Glenn, Ed.D.; Jim DeLo, Ed.D.; and especially Richard Walls, Ph.D.; and Nancy Kennedy, Psy.D. who provided thoughtful and constructive feedback while providing the support I needed at times when I needed it most.

I would additionally like to thank the wonderful people at Hawaii's Department of Education, Mahalo! Especially Joseph Acklin, Ed.D. who has been an advisor and mentor, all while being on the other side of the world. Dr. Acklin has provided me with the inspiration to become a psychologist and the encouragement to endure this task.

I would like to take this opportunity to thank Dr. Nancy Kennedy who has not only provided me with inspiration while on my committee, but also has mentored and supervised me during my clinical work throughout my doctoral studies. Dr. Kennedy has modeled and demonstrated clinical skills and provided unparalleled clinical knowledge that has been extremely advantageous to my career.

Finally, I would like to thank my family, especially my wonderful wife, Jill who has been there by my side throughout this long journey, and has never complained about the long hours of study, research, and travel that this task has involved over the past several years. 


\section{TABLE OF CONTENTS}

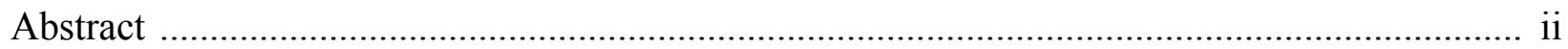

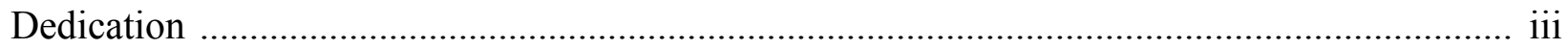

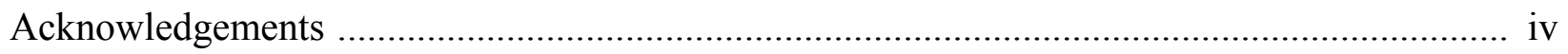

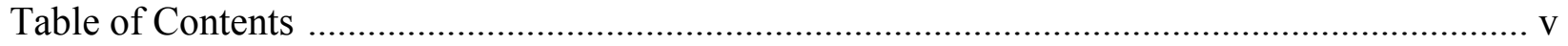

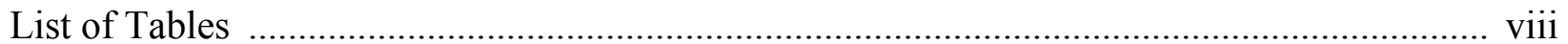

\section{CHAPTER I:}

Introduction and Review of the Literature ........................................................ 1

Development of Mental Health Services for Children and Adolescents .............. 2

Development of Mental Health Services in the School Setting .......................... 4

Utilization of Mental Health Services by Ethnic Minority Groups .................... 13

Utilization of Mental Health Services by Asian American and Pacific

Islander Populations .......................................................................... 14

Research with Asian Americans and Pacific Islanders ................................... 17

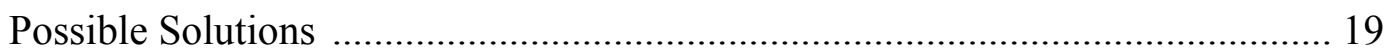

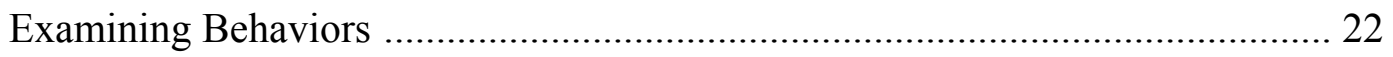

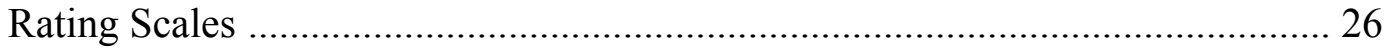

Behavior Assessment System for Children, Second Edition (BASC-2) .............. 30

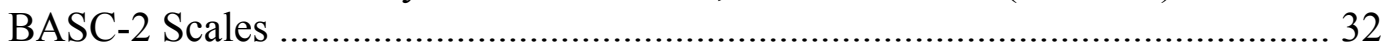

Hawaii’s School-Based Mental Health System .............................................. 33

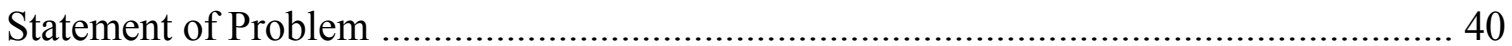

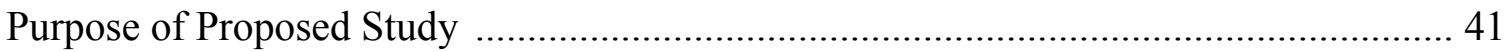

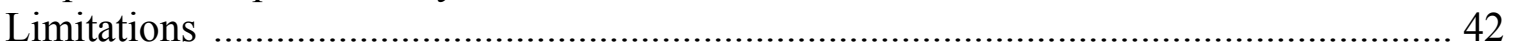

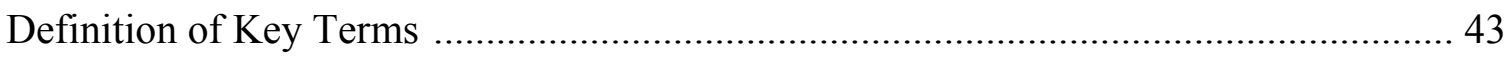

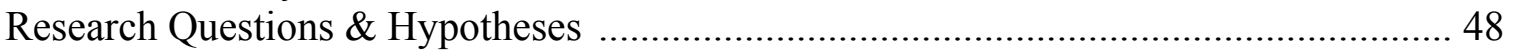

CHAPTER II:

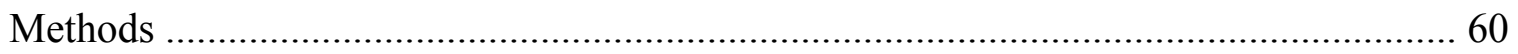

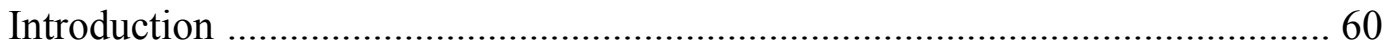

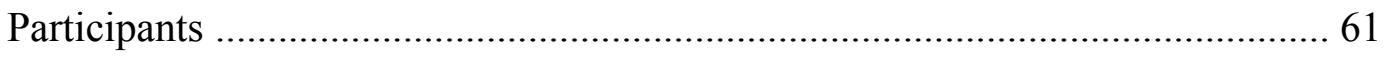

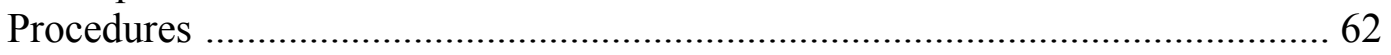

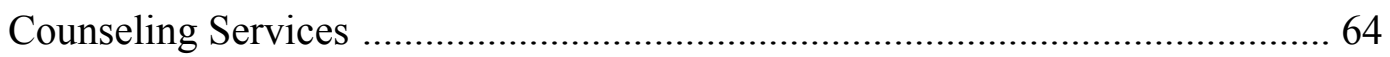

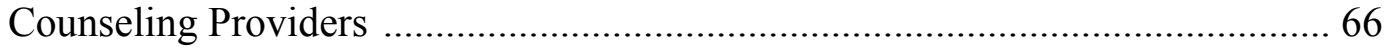

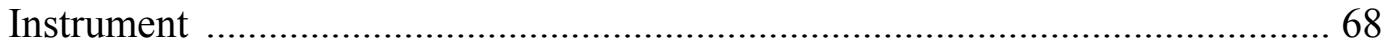

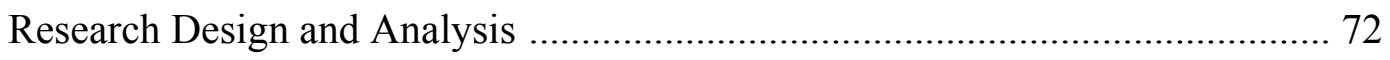

Power Analysis ....................................................................................... 74 


\section{CHAPTER III:}

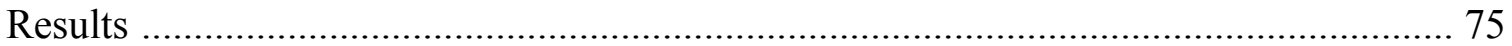

Research Question 1 .............................................................................. 75

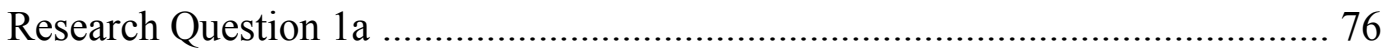

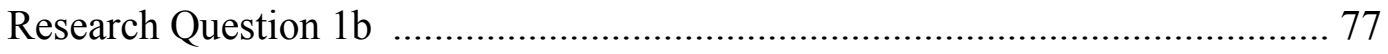

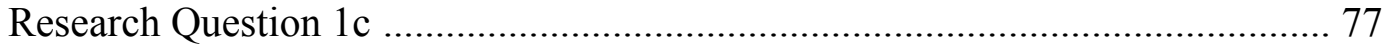

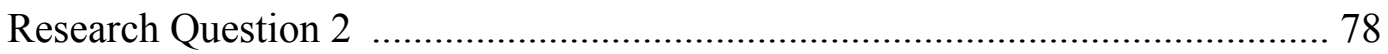

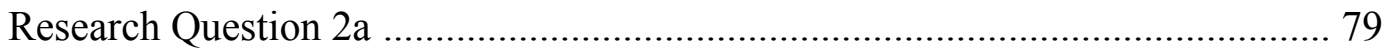

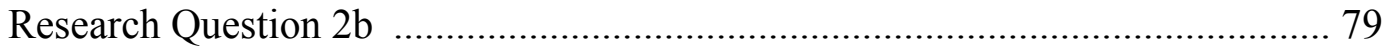

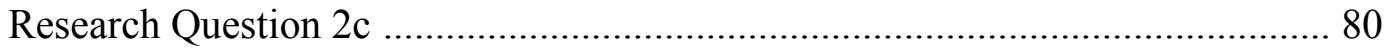

Research Question 3 ............................................................................ 80

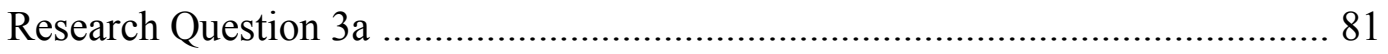

Research Question 3b ........................................................................ 82

Research Question 4 ............................................................................. 82

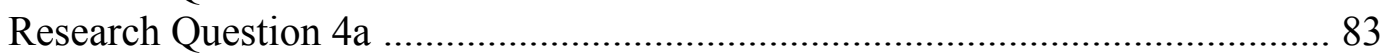

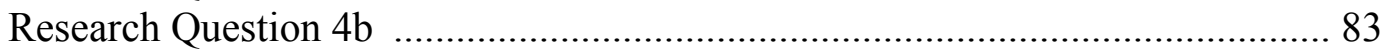

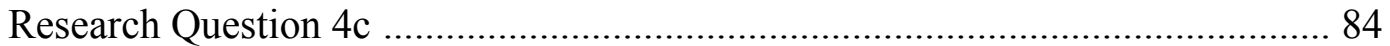

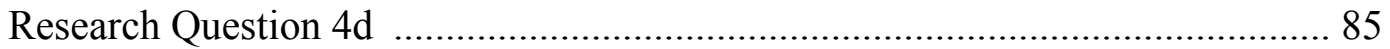

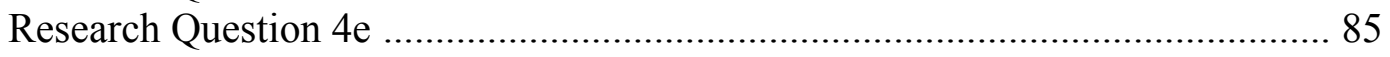

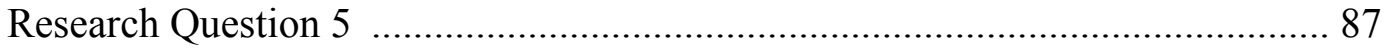

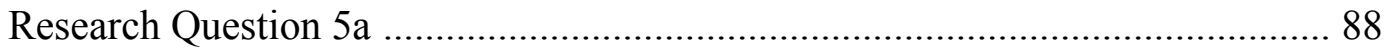

Research Question 5b ........................................................................... 88

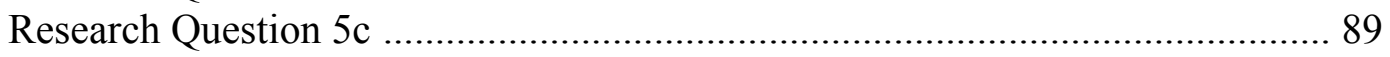

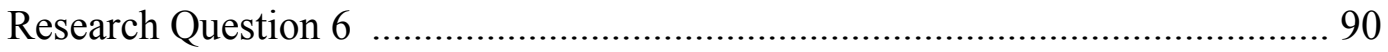

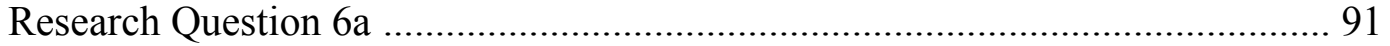

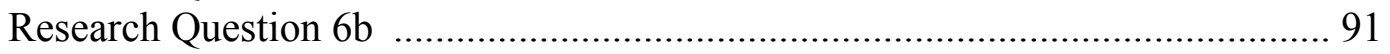

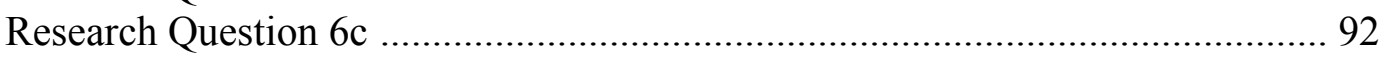

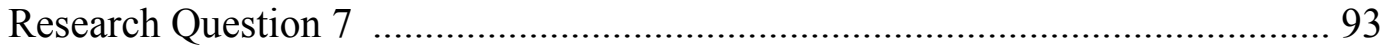

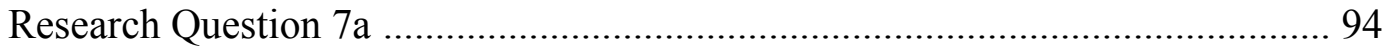

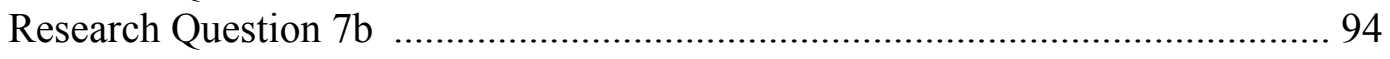

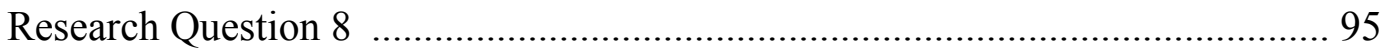

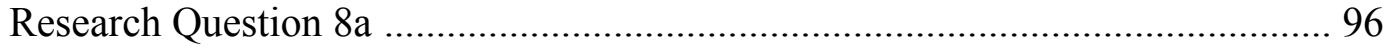

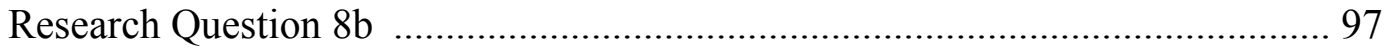

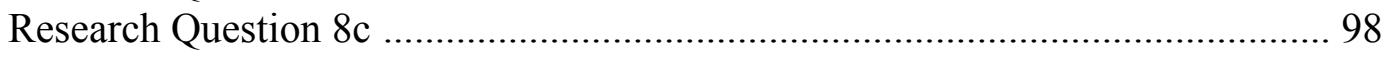

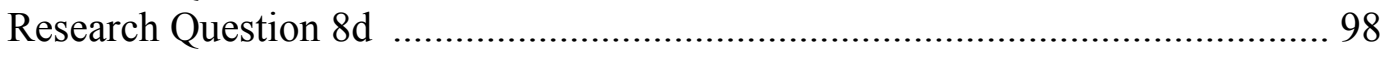

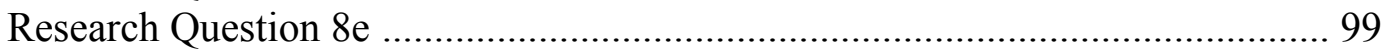


CHAPTER IV:

Discussion 101

Relationship between Student Needs and School-Based

Mental Health Services 102

Comparison of T Scores and Relationship to Intervention for Clinical Scales 103

Comparison of T Scores and Relationship to Intervention for Adaptive Scales 106

Specific Counseling Services 110

Relationship of Specific Counseling Services to Change 112

Conclusions and Implications 117

Recommendations for Future Studies 118

Limitations of Findings 119

References .... 121

Appendix A: Superintendent's Memo 127

Appendix B: Tables 131 


\section{List of Tables}

Table 1: F - Index Raw Scores Frequency Summary …..................................................... 131

Table 2: Clinical and Adaptive Composite Scale Scores ..................................................... 132

Table 3: Independent Variables for Research Questions 1, 2, 3, and 4 .................................. 133

Table 4: Predictor Variables and Criterion Variables for Multiple

Linear Regression Analyses for Research Questions 5, 6, 7, and 8 ........................... 135

Table 5: Overall Multiple Analysis of Variance (MANOVA) for

Research Questions 1, 2, 3, and 4

Table 6: One-Way Within Subjects (Repeated Measures) Analysis of

Variance for Research Questions 1, 2, 3, and 4

Table 7: Overall Multiple Analysis of Variance (MANOVA) for

Research Questions 1a, 1b, and 1c

Table 8: One- Way Within Subjects (Repeated Measures) Analysis of

Variance for Research Questions 1a, 1b, and 1c

Table 9: Overall Multiple Analysis of Variance (MANOVA) for

Research Questions 2a, 2b, and 2c

Table 10: One- Way Within Subjects (Repeated Measures) Analysis of

Variance for Research Questions 2a, 2b, and 2c

Table 11: Overall Multiple Analysis of Variance (MANOVA) for

Research Questions 3a and 3b

Table 12: One- Way Within Subjects (Repeated Measures) Analysis of

Variance for Research Questions 3a and 3b

Table 13: Overall Multiple Analysis of Variance (MANOVA) for

Research Questions 4a, 4b, 4c, 4d, and 4e 145

Table 14: One- Way Within Subjects (Repeated Measures) Analysis of

Variance for Research Questions 4a, 4b, 4c, 4d, and 4e . 146

Table 15: Research Question 5: 3 by 3 Chi-Square Matrix 147

Table 16: Research Question 5a: 3 by 3 Chi-Square Matrix 148

Table 17: Research Question 5b: 3 by 3 Chi-Square Matrix 149

Table 18: Research Question 5c: 3 by 3 Chi-Square Matrix 150

Table 19: Research Question 6: 3 by 3 Chi-Square Matrix 151

Table 20: Research Question 6a: 3 by 3 Chi-Square Matrix 152

Table 21: Research Question 6b: 3 by 3 Chi-Square Matrix 153

Table 22: Research Question 6c: 3 by 3 Chi-Square Matrix 154

Table 23: Research Question 7: 3 by 3 Chi-Square Matrix 155

Table 24: Research Question 7a: 3 by 3 Chi-Square Matrix 156

Table 25: Research Question 7b: 3 by 3 Chi-Square Matrix 157

Table 26: Research Question 8: 3 by 3 Chi-Square Matrix 158

Table 27: Research Question 8a: 3 by 3 Chi-Square Matrix 159

Table 28: Research Question 8b: 3 by 3 Chi-Square Matrix 160

Table 29: Research Question 8c: 3 by 3 Chi-Square Matrix 161

Table 30: Research Question 8d: 3 by 3 Chi-Square Matrix 162

Table 31: Research Question 8e: 3 by 3 Chi-Square Matrix 163 


\section{CHAPTER I}

\section{INTRODUCTION AND REVIEW OF THE LITERATURE}

The provision of mental health services within the educational setting is something that has been happening since the late 1800's (Pumariega and Vance, 1999). Initially, according to Porter, Epp, and Bryan (2000), these services were designed to prevent or reduce truancy or delinquency in youths. Slade (2003) found that school-based mental health services have evolved for several reasons, including an increased awareness to unmet mental health needs of children and adolescents and due to identifiable barriers in accessing mental health services for racial and ethnic minority children. Matsuoko, Breaux, and Ryujin (1997), found that Asian American and Pacific Islander populations have historically underutilized mental health services. They found in a national study that Asian American and Pacific Islander populations were three times less likely than Caucasians to use available mental health services. Yeh, McCabe, Hough, Dupuis, and Hazen (2003) found that Asian American and Pacific Islander youths with mental health problems have higher unmet mental health needs than their Caucasian counterparts. The current literature examined a variety of mental health services in the school setting and various methods and programs to meet racial and cultural minorities mental health needs, however examining the combination of these services in the schools has been overlooked. The purpose of this study is to examine the effectiveness of counseling services provided within the public school system in Hawaii. Hawaii's Department of Education has taken a unique approach to integrating services, by offering mental health services that are provided within the public school system, by the public school system. Hawaii offers a unique cultural diversity not found elsewhere, Hawaii's Department of Education has begun providing mental health services within the school setting. This integrative approach to service delivery has been in response to an 
absence of availability of mental health services for children and adolescents across the state. The basis of this study is to lend support to the effectiveness of school-based mental health services in addressing culturally diverse children and adolescents' mental health needs.

\section{Development of Mental Health Services for Children and Adolescents}

Mental health programs, according to Pfeiffer and Reddy (1998), is "any service or set of coordinated services that are intended to identify, diagnose, prevent, and/or treat behavioral, emotional, or psychological problems" (pg. 87). Mental health services for children and adolescents have evolved over time improving access to services and utilization of services. Pumariega and Vance (1999) found that mental health services have gone through clinical and cost effectiveness improvements to provide expanded mental health services to children and adolescents in their own communities. Rones and Hoagwood (2000) found that schools are the major providers of mental health services for children and adolescents. Pumariega and Vance (1999) found that school-based mental health services provide the foundation for the ultimate goal of children's mental health services, which is to fully integrate services to families within the fabric of their community and culture. The authors (Pumariega \& Vance, 1999) found that pilot programs exist, that blend community-based mental health care and school services, to provide better service access and delivery for students. Today, as services change and providers of these services become more familiar with the needs of the children, mental health services are continuing to move closer to the goal of being fully integrated within the community and culture.

Pfeiffer and Reedy (1998) and Pumariega and Vance (1999) detail the historical progression of children's mental health services in the United States. Counseling services for children and adolescents began in the United States in response to the perceived need for counseling students who were juvenile offenders, rather than incarcerating them with adults. 
Porter, Epp, and Bryan (2000) found that initially the school-based mental health services were designed to prevent or reduce truancy or delinquency in youths. In fact, Pumariega and Vance (1999) found that the very first mental health clinic geared toward children was opened at the University of Pennsylvania in 1896, focusing on school problems. Laws began to be passed relating to the best interests of children, including the compulsory education law passed in 1860 , followed by the establishment of child abuse laws in the 1880's, and the juvenile court system, which was established in the early 1890’s (Pumariega \& Vance, 1999). As noticeable increases in the severity of diagnostic issues with children and adolescents became more prevalent so did the use of psychotropic medications (Del Mundo, Pumariega, \& Vance, 1999). The development and funding of child guidance clinics throughout the United States was initiated in 1922, after the Commonwealth Foundation commissioned a study in response to interdisciplinary teams being used in Chicago and Boston in the early part of the 1900's (Pumariega \& Vance, 1999). These programs later would be the foundation of the first child psychiatry programs in the nation. Some of these child guidance clinics, according to Pumariega and Vance (1999), were created to serve school districts, utilizing a multidisciplinary approach to treatment.

Contemporary changes in mental health services for children and adolescents have evolved over time, with the development of several national policy initiatives (Pfeiffer and Reddy, 1998). According to Pumariega and Vance (1999), the 1970s and 80s began a move toward a hospital-based model of mental health services. The authors found that this shift toward hospital-based programming left the mental health centers neglected, understaffed, and under funded. With mental health centers being less effectively utilized for providing adequate care for children and adolescents, society was forced to develop more creative ways to provide appropriate care to children and adolescents. According to Pumariega and Vance (1999), the 
1980s brought the development of community-based systems of care. These programs were developed and based on the Children and Adolescent Service System Program (CASSP) model (Pumariega \& Vance, 1999). The CASSP initiative, according to Pumariega and Vance (1999), was modeled on the ideas and framework presented by Stoul and Friedman regarding community-based care for emotionally disturbed children. The CASSP model, according to the authors, advocates for interagency coordination among all of the child-serving agencies in the provision of mental health service for children and related family support services. These community-based programs are representative of an outreach model, where the services and the providers are implementing the mental health services within the client's natural environment. These services are sometimes also referred to as "wrap around" (p. 376) services and are unconventional in nature, due to the service being provided within the client's natural setting home, community, and school settings (Pumariega \& Vance, 1999). The CASSP model, according to Pfeiffer and Reddy (1998), not only called for culturally competent mental health services, but also called for these services to be available in the school setting. The CASSP model was one of the first national initiatives that specifically called for mental health services to be provided in the schools, setting the groundwork for future developments in school-based mental health services.

\section{Development of Mental Health Services in the Schools}

With mental health services being protracted in traditional settings, and increasingly found in non-traditional settings, there was a need for children's mental health services to be expanded beyond the walls of the traditional counseling setting into other settings. This gap between what is needed and what is available in mental health services for children and adolescents, created a need for the educational system to become much more involved in the 
treatment of children and adolescents. Heneghan (1997) in one study found that school directors report higher rates of mental health problems and learning disabilities than chronic medical problems or physical disabilities. According to Ringeinsen, Henderson, and Hoagwood (2003), schools play a critical role in the delivery of mental health services to children and adolescents, and children and adolescents with mental health needs are more likely to receive treatment when mental health services were offered in the schools, rather than in the community. Pumariega and Vance (1999) state that, "the centrality of school in the life of the child makes it the ideal center for a comprehensive community-based system of care" (p. 374). The authors found that utilizing the schools provides a place of entry for services that are non-stigmatizing, a naturalistic setting to observe behavior (particularly by educators who have an expertise in normal development), and the ability to integrate interventions into the natural environment. There has been an increase in the utilization and need for children's mental health services, due to the increase in prevalence of child and adolescent mental illness (Pfeiffer \& Reedy, 1998; Pumariega \& Vance, 1999).

Schools are becoming increasingly involved in the treatment of mental health conditions of students, but funding for these programs has diminished (Pumariega \& Vance, 1999). Robinson and Rapport (2002) found that the combination of mental health and educational services offers many benefits to children and families. As changes in mental health services were occurring, Public Law 94-142 (Slade, 2003; Aldman \& Taylor, 2000; Pfeiffer \& Reedy, 1998; Pumariega \& Vance, 1999) was implemented which was later re-authorized as the Individuals with Disabilities Education Act (IDEA) (Pumariega \& Vance, 1999). Public Law 94-142 and IDEA provide legal avenues for children and adolescents to access an appropriate education regardless of handicap condition or disability (Pumariega \& Vance, 1999). IDEA 
recently was re-authorized again leading to additional reshaping of the form of services provided in the school setting (Pumariega \& Vance, 1999). According to Casat, Sobolewski, Gordon, and Rigsby (1999), federal acts such as Section 504 of the Rehabilitation Act of 1973, mandate schools to provide a free and appropriate education and to ensure the removal of barriers to learning opportunities to all students regardless of their disability. The passages of these acts and others like them have forced the schools to deal with an increasing number of mental health issues in the school setting. According to Weist, Myers, Danforth, McNeil, Ollendick, and Hawkins (2000), these policies reflect a trend toward future expanded school mental health services.

Schools were faced with the difficult task of having to meet children's mental health needs in the school setting without the funding necessary to carry this out. Schools' budgets for these types of programs were not increased proportionally in order to provide additional mental health services in the school setting (Casat et al., 1999). Weist et al. (2000) found in a survey of school administrators, that school personnel have realized a need for alternate services, and welcome additional mental health services being provided in the school setting. Ringeisen, Henderson, and Hoagwood (2003) found that effective school-based mental health care would only result from a marriage of the mental health and school systems. Developing this collaboration of systems has not been an easy task (Weist et al., 2000). Initially trying to provide mental health services in the school setting overwhelmed school systems (Aldman \& Taylor, 2000). Schools were designed to provide educational services, not mental health services. Instead of merging systems and providing mental health services themselves, schools began to work collaboratively with private providers of mental health services in the community, utilizing a multidisciplinary approach to delivery of services. This brought private mental health agencies 
into the school setting to provide mental health services within the school setting. This was a relatively new approach to service delivery within the school setting. This model of utilizing private mental health agencies within the school setting to provide mental health services is seen today on a regular basis.

Schools began to look outside of their own available services to access assistance from private and public human service agencies. According to Adelman and Taylor (2000), schools are mandated to educate, not provide mental health services, and therefore schools often view providing these services as taking resources away from their primary mission. Schools began to make available expanded school mental health services by developing collaborative agreements with outside private and public agencies (Weist et al., 2000). By entering into these agreements, schools began providing expanded school mental health services, allowing schools to provide a full range of mental health services within the school setting (Weist et al., 2000). The authors found an emerging issue with expanded school mental health services; these services are often times not developed based on a careful analysis of the needs of the students. The authors also point out that programs need to be developed which document that services are in fact meeting the needs of students (Weist et al., 2000). Pumariega and Vance (1999) found that this fragmentation of care could result in shifting responsibility of providing services and costs of those services back and forth across child serving agencies and school systems. This appears to be in part due to the disconnection between private agencies and public school systems. These systems are not motivated to provide services by the same entities, causing various problems in providing services within the school setting.

Casat et al. (1999) found that improving educational quality and outcomes in the public schools has been an intensely pursued agenda in the United States. Nabors, Weist, Tashman and 
Myers (1999) found that expanded school mental health programs offer a unique opportunity for collaboration between traditional school personnel and clinical mental health services. These expanded school mental health service programs provide a range of mental health services in the school setting. According to Weist et al. (2000) these services include assessment, prevention, case management, and treatment services for students in regular and special education services. The authors did not examine programs that provided mental health services by the school districts. Pumariega and Vance (1999) found that advanced models of service provide several levels of services to include all students in the school, not just students labeled with a disability. The authors found that these programs included primary preventative services for all students, secondary prevention services for at-risk students, and formal mental health services to be delivered during the school day.

In today's society, mental health services occur in a wide variety of settings. Traditional mental health services, often referred to as counseling or therapy, where the client comes to the clinician in the office setting are quickly becoming the exception as opposed to the norm for children and adolescents. Rones and Hoagwood (2000) found that schools are the major providers of mental health services for children and adolescents who receive these types of services. Slade (2003) found that "schools in the United States are becoming increasingly involved in the delivery of mental health services to children" (p. 382). Ringeisen, Henderson, and Hoagwood (2003) found that schools play a critical role in the delivery of mental health services for students. Carton and Weiss (1994) found that students with mental health needs identified in school are more likely to enter and receive treatment when mental health services are offered in school rather than when services are offered within the community. Pumariega and Vance (1999) found that the future of our children is brighter due to the promise of success 
that school-based mental health services have to offer. Slade (2003) stated that, "the number of school-based and school-linked health centers increased from 0 in 1970 to 948 in 1997, with approximately two-thirds of them providing mental health services" (p. 382). There are more students in today's public school system identified as having emotional disabilities than ever before, yet these needs are still underserved (Slade, 2003). Rones and Hoagwood (2000) found that although one-fifth of children in the United States have a diagnosable mental disorder, only about $16 \%$ of them ever receive any type of mental health service. Rones and Hoagwood (2000) also found that of the $16 \%$ of children who receive mental health service, the overwhelming majority of them (70\% to $80 \%$ ) receive their services within the school setting. U.S. Department of Education (2001) estimates that less than 1\% of all children are identified as having an emotional disturbance and receive special education or related services under IDEA.

Today, schools are being required to shoulder more of the responsibility of providing ongoing counseling services to students within the school setting. Providing mental health related services in the school setting is not new to the field of psychology and education (Rones \& Hoagwood, 2000). School is the logical and important place to provide mental health service interventions, however creating access to these services is not a simple process (Ringeisen et al., 2003). Slade (2003) found that under the 1975 Education for All Handicapped Children Act schools are required to provide access to mental health services whenever mental health treatment is part of a student's Individual Education Program (IEP). Schools, according to Knitzer, Steinberg and Fleisch (1991), are often reluctant to officially refer students for counseling. The authors found that schools are ultimately financially responsible for these services if they are included in a student's IEP. Instead, Knitzer et al., (1991) found that schools generally will request that parents seek out counseling services on their own, where the authors 
also found that little collaboration is present between the school and the mental health provider. Schools have traditionally been staffed with school counselors, school psychologists, and even social workers to assist students when they are having emotional or behavioral difficulties. These services are typically provided on a very short-term basis, crisis intervention, as well as vocational and educational planning for hundreds of students within the school setting (Porter et al., 2000). When more intense psychopathology is present, a referral to an outside agency or clinician has typically been made. Traditionally, according to Porter et al. (2000), the effectiveness of school-based mental health services is dependant on a collaborative relationship between schools and outside mental health agencies. Initially, the authors found that, outside agencies were welcomed as resources to complement or enhance the services provided by the schools. As time progressed, the authors found that, school personnel began to have ambivalent and even jealous feelings toward the mental health professionals, mainly due to their freedom from administrative responsibilities. Porter et al. (2000) note, that a worthy alliance that had expanded resources for students was seriously damaged.

Fiscal concerns are an increasing problem in mental health service delivery. Pumariega and Vance (1999) found that although mental health budgets for children and adolescent services have increased, a large percentage of those funds are utilized for inpatient and residential care. When outside agencies provide services to students within the school setting, managed care often times influences how and when services are provided (Porter et al., 2000). The staff of the private or public mental health agency provides consultation to the school staff, under the provision of billing Medicaid for these services (Knitzer et al., 1991). Traditional school-based mental health services combine school personnel and outside agencies to meet students' needs. One difficulty with these programs is the instability of funding for mental health programs in the 
current managed care environment, "do more with less" (Weist et al., 2000, p. 262). Third-party payers, under current managed care guidelines, are more reluctant to authorize therapy services for extended periods of time, even when the necessity of these services is clinically indicated, allowing, according to Knitzer et al. (1991) for short-term treatment. According to Slade (2003), many schools even enter into agreements with state Medicaid offices in order to receive financial reimbursement for mental health services, an option allowable under federal law (Knitzer et al., 1991). Slade (2003) also found that another significant source of public funding is through Title V Maternal and Child Health Block Grant program, which provides matching funds to schools for a variety of services. Other sources of funding, found by the author, that are tapped into by schools are grants from private and public sources, and out-of-pocket payments from students. Schools do not seem to seek reimbursement from private health insurance companies. The need for improved and innovative programming in the school setting is necessary.

As can be seen in the above compilation of literature, mental health services for children and adolescents have evolved over time inside and outside of the school setting. A number of studies have found that locating the point of service in the schools is the most effective way to meet the ever changing needs of students. The manner in which this has been done is subject to great debate, and effective school-based mental health services are provided in a variety of ways. Outside agencies, both public and private, have traditionally been the provider of mental health services in the school setting. Porter, Epp, and Bryan (2000) discuss the use of school-based mental health providers, to increase the opportunity to expand the quality and quantity of mental health services needed by many students. School-based mental health providers are mental health service providers (i.e. social workers, clinical psychologists, counselors, etc.) that are employed by the school districts to provide additional mental health services within the context 
of the school setting. The major difference in these services is not the service provided by the individual provider, but instead is the manner in which the service is paid for and managed. School-based mental health services are paid for by the schools and their activities are managed by the schools. This revolutionary idea, according to Porter et al. (2000), theoretically reduces or limits the competing ideas between school personnel and agency personnel. These school-based mental health services, according to Porter et al. (2000), are particularly effective when they work as a team and provide a continuum of care and integrate services in the school setting. According to the authors, these school-based mental health professionals are able to bridge the discontinuity in mental health services between schools and outside agencies. According to the authors, these school-based mental health services offer a link to the educational setting and outside community resources where additional specialized services are available.

These services, although improved, are not without problems. Porter et al. (2000) found that although providers are employed by the school districts there continue to be "turf issues" (p. 1104). There are also differences in clinical approaches, and the on-going issues related to interpreting school procedures and policies. An additional problem found by the authors was an increased opportunity for students, parents, and even school personnel to engage in "splitting" (p. 1104) (putting one professional against the other) behaviors. These problems although present, are less so than the problems with older models of school-based mental health services. The problems can be avoided or minimized with mutual respect, ongoing collaboration, and increased communication. The current literature does not address racial or cultural diversity issues in providing school-based mental health services. School-based mental health services have been studied in various settings, but the current literature does not address cultural diversity when examining these services. 


\section{Utilization of Mental Health Services by Ethnic Minority Groups}

There has been increased attention in the literature, over the past two decades, regarding the problem of utilization of mental health services by members of ethnic minority groups. Statistically, minority groups have always underutilized mental health services and not much has been done to improve this until recently. Snowden and Cheung (1990) noted that minority groups have continually been underrepresented and have underutilized mental health services. Pumariega and Vance (1999) found that minority populations have traditionally been underserved by mental health services. Sue, Fujino, Hu, Takeuchi, and Zane (1991) found that all ethnic minority groups tended to drop out of treatment quickly. Okazaki (2000) found that past studies have indicated minority groups tend to underutilize outpatient and inpatient mental health services and at the time of admission are more likely to be diagnosed with psychotic disorders than their Euro-American counterparts. Researchers (Okazaki, 2000) have dismissed the notions that minority groups do not utilize mental health services as often because they have fewer problems or because they are any healthier than their white counterparts. It appears that systemic problems exist that have always contributed to the underutilization of mental health services by minority groups. Mental health services are not readily available to minority groups, and the services that are available often times are not culturally sensitive to the clients' needs.

Lau and Zane (2000) found that the underutilization of mental health services by minority populations is directly correlated to the service delivery of those mental health services for the minority populations. Ethnic minority groups appear to underutilize mental health services because they do not seek services and therefore do not access the available services. Bell (2003) found that ethnic minority groups are less inclined than whites to seek treatment from mental health specialists and more likely to seek treatment from informal sources such as clergy or 
traditional healers. These informal services are not widely viewed as mental health treatment providers. Chow (2002) indicated that underutilization of services by ethnic minority groups, is evidence of a barrier to services use rather than an absence of needs. Researchers have been focusing on how to overcome this barrier to treatment for the past several years. This barrier to treatment begins with these clients not seeking assistance for their emotional concerns.

\section{Utilization of Mental Health Services by Asian American and Pacific Islander Populations}

In nearly all cultures mental health services are typically available in a variety of settings including community mental health centers, clinical and private hospitals, and private practice settings. These services are designed as a one-stop-shop to serve a wide variety of clients and their multitude of complex needs. Mental health service centers typically have an open-door approach to service, and attempt to provide treatment to all clients, regardless of cultural or ethnic background. Unfortunately, Asian American and Pacific Islander populations have been found to significantly underutilize mental health services when compared to Euro-Americans. Matsuoka, Breauz, and Ryujin (1997) found that the proportion (.41\%) of Asian Americans and Pacific Islanders utilizing mental health services is a third of the proportion (1.24\%) for EuroAmericans. When they examined this data closer, they found that across all types of services and all types of facilities (outpatient services, inpatient services, residential treatment, and partialhospitalization treatment) that Asian Americans and Pacific Islanders are much less likely to utilize mental health services. Unfortunately, the current literature has not examined schoolbased mental health services for Asian American and Pacific Islander populations. It is unclear from the current literature if this approach of providing mental health services in the school setting has been looked at specifically for minority populations. 
McCabe et al. (1999) as cited in Yeh, McCabe, Hough, Dupuis, and Hazen (2003) found that Asian American and Pacific Islander populations were underrepresented in public mental health services by about one half as compared to what would be expected given their representation in the population. In order to determine if an ethnic population is underrepresented, the percentage of a given population enrolled in mental health treatment is compared to other ethnic populations enrolled in treatment. Asian American and Pacific Islander populations have always shown a marked decrease in the mental health services they access. Matsuoka, et al. (1997) found in their national study that Asian Americans and Pacific Islanders were three times less likely than their white American counterparts to use available mental health services. Yeh et al. (2003) found that Asian American and Pacific Islander youth populations, with mental health problems, have statistically significant higher unmet mental health needs as compared to Non-Hispanic Whites $(71.8 \%$ compared to $30.7 \%$ when $p<.0001)$. These statistics are staggering and when viewed from a national policy standpoint they are troubling and an indication of the need for change.

Sue et al. (1991) cite the President's Commission on Mental Health as an early source of recognition that this problem of underutilization of mental health services exists. Researchers began questioning this phenomenon of underutilization of mental health services years ago, and attempts have finally begun to correct this problem. Unfortunately longitudinal studies take a very long time to complete and data is only now being published about these studies.

This issue of underutilization of mental health services by Asian American and Pacific Islander populations has been identified as a major problem and must be addressed. This problem will most effectively be addressed by examining the factors that contribute to the underutilization of mental health services by these Asian American and Pacific Islander 
populations. The underutilization of services by Asian Americans and Pacific Islanders is now being viewed as a barrier to services for these groups. This is a paradigm shift in how this problem is viewed. Sue and McKinney (1975) as cited in Chow (2002) noted that these barriers to service indicate that existing services are not appropriate to serve Asian American and Pacific Islander populations. Previously, this problem was viewed as the minority groups were not using the available services. The solution was geared toward how to get Asian Americans and Pacific Islanders to access the current services provided. Today, it appears that the research has determined that the available services are not necessarily the most appropriate services, and therefore the development of new, more innovative services is necessary. These services must perform outside the function of traditional mental health services and meet the specific needs of Asian American and Pacific Islander populations.

In the state of Hawaii this issue is at the very forefront of discussion for policy makers and service providers. The U. S. Census Bureau (2000) reports that according to the 2000 U. S. Census data there are 617,407 Asian, Native Hawaiian, and Other Pacific Islander's living in the state of Hawaii, making up 51\% of the total population. Those of Asian decent make up $41.6 \%$ of the population alone. According to the Hawaii State Department of Health (2003), the Behavioral Health Administration's mission is to provide leadership to monitor, promote, protect, and enhance the well being of all of Hawaii's people. The Hawaii State Department of Health (2003) goes on to explain that this is done by assuring the availability and coordination of mental health and substance abuse services to address the needs of adults and children in Hawaii. $\mathrm{Hu}$, Snowden, Jerrell, and Nguyen (1991) state that achieving the objective of providing adequate mental health services to the entire population requires an understanding of factors that affect utilization of mental health services, especially for minority populations. The authors did 
not examine how school-based mental health services would address the needs of minority students. Minority populations, particularly, those of Asian and Pacific Islander decent, do not access mental health services within the community, due to an attached stigma of those services. HDOE has developed their program to have the mental health services available within the school setting. The students do not need to actively seek out these services in order to access them; the services instead come to them. The parents do need to give consent to services, which is a much smaller hurdle than attempting to bring students into outpatient clinics to access services.

Research with Asian Americans and Pacific Islanders

Conducting research with minority populations poses special consideration of cultural and ethnic issues. Understanding the factors involved has been a major problem in conducting research with ethnic and racial minority groups. Palafox, Buenconsejo-Lum, Rinklon, and Waitzferlder (2002) discuss the necessary shift to a culturally competent model of conducting research with indigenous Pacific Islander populations. The authors cite difficulties associated with conducting accurate research using a Western model. These difficulties include common beliefs held by Western researchers that it is their fundamental right to pursue and acquire knowledge (Palafox et al., 2002). Many indigenous Pacific Islander populations historically believe that knowledge is a protected entity and have developed resentment for research. If research is being conducted in a manner that is not conducive to the collection of accurate data, a change is necessary in the very method used to collect data and conduct research. Palafox et al. (2002) call for socially and culturally responsible research to be conducted when dealing with Asian American and Pacific Islander populations. The authors raise the questions of increased cultural sensitivity and cultural understanding when attempting to conduct research with Asian 
American and Pacific Islander populations. Palafox et al. (2002) cited that the lack of cultural sensitivity and cultural understanding has lead many Asian American and Pacific Islander groups to have strong feelings of disenchantment and resentment toward the Western research process. As researchers continue to conduct both large and small-scale research on indigenous populations, they will need to significantly improve the methods of data collection and research to include an improved culturally sensitive approach. Without the cooperation of the Asian American and Pacific Islander population Western researchers will likely find it very difficulty to study these groups and to make the necessary changes to the mental health services that are provided.

As presented in the above review of the literature, collecting valid data for research of ethnic minority populations is a difficult task. Asian American and Pacific Islander populations are typically not interested in research and are generally skeptical of anyone conducting research. New and innovative approaches to conducting research must be pursued in order to accurately assess the current state of mental health services for Asian American and Pacific Islander populations.

For Asian Americans and Pacific Islanders, language is a very serious barrier to treatment. According to the U. S. Census (2000) data, $73.4 \%$ of the population $(832,226$ people) in Hawaii speak only English, 23.6\% of the population (267,157 people) speak either an Asian or Pacific Island language, and $11.9 \%$ of them (134,782 people) report that they speak English "less than very well." These numbers indicate that language is a potentially enormous barrier to treatment of the Asian American and Pacific Islander population in Hawaii. In many cases, as cited in Yeh et al. (2003), if an Asian or Pacific Islander client is seeking services they may have difficulty finding one that speaks their language. The U. S. Census (2000) also indicates that only 
$8.4 \%$ of the population in the state of Hawaii has earned a graduate or professional degree, the level of education necessary to provide most mental health services. Individuals trained to provide mental health services are often not available in all areas to provide this treatment to minority clients.

According to Leong (1994) knowing how Asian American and Pacific Islander populations utilize various mental health services is important information that would be useful in policy decisions, treatment planning, and outreach efforts. The development of appropriate mental health services for Asian American and Pacific Islander populations has recently begun an implementation phase in the United States. Studies have been conducted in large urban areas to identify contributing factors and begin the development of mental health service systems that meet Asian American and Pacific Islander population needs. According to Yeh et al. (2003) understanding the barriers experienced by various minority groups is a critical first step to reducing documented disparities in mental health service use by ethnic minorities.

\section{Possible Solutions}

Many of the barriers to treatment for Asian Americans and Pacific Islanders in Hawaii relate directly to ethnicity and culture. These groups typically have great difficulty locating and gaining admission to appropriate mental health services, they are stigmatized by the possibility of accessing these services and they often times are limited by financial and language barriers. Several researchers have begun to develop possible solutions to address these issues for Asian Americans and Pacific Islanders. Chow (2002) advocates for the further development of ethnicspecific services (ESS) to better meet the needs of these populations. Sue et al. (1991) examined the use of a "culturally responsive" (p. 534) strategy including the increased training of personnel to work with culturally dissimilar clients, the recruitment and employment of more 
bilingual and bicultural therapists, and the development of parallel services that are devoted specifically to ethnic minority clients. Education is faced with many of the same dilemmas, attempting to provide educational services to culturally dissimilar students. Hawaii has combined the two, providing educational and mental health services all under one umbrella of service. It appears that developing culturally specific or culturally responsive models of treatment or education is still in the infancy stage of development. This is surprising considering that differences in race and ethnicity have been issues of discussion and distrust for centuries. The helping professions have taken a very long time to examine this issue, but it appears that we are headed in the right direction at this point.

When researchers view the development of new and innovative services for Asian American and Pacific Islander populations, they first examine the problems with the current services and have made attempts to change what already existed. The problems that were identified spoke mainly to the cultural insensitivity of the current services. Bell (2003) notes that the major problem with the Western origins of mental health services is that they discount other cultures' understanding of mental health and mental wellness. Making changes only to existing services does not meet the needs of the clients. Researchers are forced to develop new services that are individualized and specifically designed to meet culturally diverse clients.

Insight was provided for a set of strategies developed out of a study done in 1977. Sue et al. (1991) cite the "culturally responsive" model of changes that came out after a large study by Sue in 1977. These changes included many of the things that are considered, even today, in multicultural training courses. Sue et al. (1991) cited the strategies for change to include training of personnel to work with culturally dissimilar clients, the employment of more bilingual and bicultural mental health workers, and the establishment of parallel services specifically designed 
for ethnic minority clients. As these strategies were reviewed and explored with several minority populations, the researcher received mixed results. It is unclear whether these strategies fully meet the needs of ethnic minority clients. Sue et al. (1991) cited that it is uncertain that the current services provided are adequate to meet the needs of these groups. Although the results appear mixed, this appears to be the most conclusive evidence of how to better meet the needs of culturally diverse populations.

Another possible solution to this problem of underutilization of mental health services by Asian American and Pacific Islander populations is also provided. This solution focuses much more on the services provided by the system as a whole rather than an individual service provider. Chow (2002) advocates for the further development of Ethnic-Specific Services (ESS) specifically designed for Asian American and Pacific Islander populations. Although this model appears very different in nature it remains similar to the "culturally responsive" strategies in spirit. The Ethnic-Specific Services model is the delivery of traditional mental health services (i.e. outpatient therapy, psychiatry, assessment, etc.) in a culturally appropriate manner to meet the specific needs of the clients within the Asian American and Pacific Islander community (Chow, 2002). These services are infused into the community where the clients that need them can access them easily. Mental health professionals who are also of Asian American and Pacific Islander descent provide all of the services to the potential clients and operate the ethnic-specific service centers. This is also true in Hawaii, where many of the providers of educational and mental health services are of various cultural descents. This ethnic-specific service system provides solutions to many problems found in the current model of providing services to Asian American and Pacific Islander populations. 
Chow (2002) found through her analysis of existing programs that significant improvements exist in mental health service utilization and outcomes, but notes that the services are not perfect or complete. The existing services that are classified under this model currently only provide a limited amount of mental health services. The results of this study are hopeful and the ethnic-specific service described will likely be expanded to provide a wide array of mental health services to Asian American and Pacific Islander groups within their communities. According to Chow (2002), these services would include, but would not be limited to, outpatient therapy, case management, vocational rehabilitation, residential care, and inpatient hospital care. The author did not include school-based mental health services as a mode of service delivery. This is an area that has been neglected in the literature for providing mental health services to culturally diverse populations.

Future researchers and scholars need to be aware that this problem of minority populations underutilizing mental health services has existed for a very long time. Unfortunately, professionals have known about this problem for a very long time also, yet little has been done to change this until recently. Professionals cannot allow the wheels of change to move so slowly. The President's Commission on Mental Health in 1978 as cited in Sue et al. (1991) identified many of the problems that we know exist in minority populations and their utilization of mental health services. We cannot allow change to take twenty-years to occur and yet today we still have a long way to go in order to perfect the system. Researchers today need to be aware of these issues so that history does not repeat itself.

\section{Examining Behaviors}

Emotional and behavior problems experienced by children and adolescents of all races and ethnicities have been studied extensively by researchers in the fields of psychology, 
psychiatry, and education. These emotions and behaviors can be examined in a multitude of ways. Two broad categories that have significance are Internalizing Disorders and Externalizing Disorders. Although the Diagnostic and Statistical Manual of Mental Disorders, Fourth Edition, Text Revision (DSM-IV-TR) (2000) does not specifically classify disorders into internalizing and externalizing, these are broad categories that are readily accepted in the field of psychology. Many of the disorders found in childhood and adolescence can be grouped under the headings of internalizing and externalizing. Examining emotions and behaviors experienced in childhood and adolescence is important for several reasons. Compton et al. (2002) found in their review of recent studies that there is evidence that suggests internalizing disorders experienced by children and adolescents are associated with early onset of substance use disorders. Farmer et al. (2002) found that externalizing disorders are precursors to adolescent substance use disorders. A comprehensive approach to examining emotions and behaviors is essential to providing appropriate interventions and treatment for children and adolescents.

Hyperactivity, aggression, conduct problems, and attention problems, according to Friedberg and McClure (2002), are all common externalizing disorders found in childhood and adolescence. Chambless and Ollendick (2001), as cited in Chambless, Baker, Baucom, Beuler, Calhoun, et al. (1998) and Spirito (1999), found in their review of the current literature the most efficacious treatments for children with oppositional defiant behavior to be several forms of cognitive behavior therapy and family interventions. Chambless and Ollendick (2001) also found for children with attention and hyperactivity problems, behavioral parent training and behavioral modification interventions to be most effective. Franklin, Harris, and Allen-Meares (2006) found that a multimodal approach to treatment, including pharmaceutical and behavioral 
interventions, including cognitive behavioral therapy, teacher, and family centered approaches, across settings to be most effective.

Friedberg and McClure (2002) recommend utilizing a behavioral checklist or rating scale to assessment these disruptive behaviors problems, including hyperactivity, aggression, conduct problems, and attention problems. Farmer et al. (2002) found in reviewing a study conducted in 1991 by Lochman, Coie, Underwood, and Terry that group and individual therapy in the school setting indicated significant reductions in aggression and improved social behaviors as measured by the Teacher Behavior Checklist. Farmer et al. (2002) found in reviewing a study conducted in 1989 by Lochman, Lampron, Gemmer, Harris and Wyckoff that group therapy with teacher consultation in the school setting exhibited improvements in disruptive and aggressive behaviors. The authors examined several additional studies including one conducted in 1999 by the Conduct Problems Prevention Research Group that exhibited improvements in peer interactions and behavioral improvements after group and individual therapy. Friedberg and McClure (2002), detail a cognitive-behavioral treatment approach implemented when dealing with disruptive behaviors in children and adolescents. The authors note that disruptive children are not typically motivated to change, and engaging them in treatment is the first step to success. Behavioral skills are taught to the child and the parents or caregivers, followed by an increased cognitive focus while integrating behavioral interventions.

Anxiety, depression, and somatic complaints are common when working with children and adolescents (Friedberg \& McClure, 2002). Chambless and Ollendick (2001) found in their review of current studies by Chambless, Baker, Baucom, Beuler, Calhoun, et al. (1998) and Spirito (1999), cognitive behavioral therapy to be probably efficacious in the treatment of depression and anxiety disorders. The studies reviewed by Chambless and Ollendick (2001) 
included a wide range of anxiety and depressive disorders. No treatments were found to be wellestablished for the treatment of childhood depression and anxiety, but the above cited studies were found to be probably efficacious. Somatic complaints are studied much less in the current literature. Franklin, Harris, and Allen-Meares (2006) found somatic complaints to be associated more with grieving children and adolescents than other mental health diagnoses.

Friedbert and McClure (2002) recommend utilizing self-report, parent rating scales, and teacher rating scales when assessing these symptoms of internalizing behavior. Compton et al. (2002) found in reviewing two specific studies (Jaycox, Reivich, Gillham, \& Seligman, 1994; Weisz, Thurber, Sweeney, Proffitt, \& LeGagnoux, 1997) short-term cognitive-behavioral interventions were more effective than no treatment in reducing symptoms of depression as measured by rating scales for childhood depression. Treatment approaches vary; Friedberg and McClure (2002) recommend a cognitive behavioral approach when treating internalizing disorders that include individual and family involvement.

Emotional and behavioral difficulties have a variety of intricate aspects that should be assessed from multiple viewpoints. Adolescent and childhood behaviors, both internalizing and externalizing, are typically examined by the use of some rating scale. These rating scales are completed by a parent, teacher, or someone else who has regular contact with the child in a structured environment. There are some scales designed to be completed by the child. Rating scales, according to Reynolds and Kamphaus (2004), are particularly well suited for recording specific observable behaviors. When examining childhood and adolescent emotional and behavioral concerns, previous researchers have utilized a wide variety of rating scales to assess concerns. Some rating scales have begun to rate adaptive behaviors in addition to maladaptive behaviors. Farmer et al. (2002) also found in a study conducted in 1998 by Walker, Kavanagh, 
Stiller, Golly, Serverson, and Feil that group and individual therapy produced improved adaptive behaviors and less maladaptive behaviors, as measured by the teacher's ratings of adaptive behavior on the Child Behavior Checklist. As can be seen from the previous literature, there is great importance in identifying child and adolescent emotional and behavioral concerns. Identification of these concerns is the first step to appropriate intervention.

Literature that exists on the study of Adaptive Behaviors appears exclusive to Autism Spectrum disorders and Mental Retardation. Franklin, Harris, and Allen-Meares (2006) found that when a clinician finds that mental retardation or other developmental delays exist it is beneficial to rate behaviors that may be out of the child's developmental age range. There are a variety of measures used to rate adaptive behaviors, including the Vineland Adaptive Behavior Scale (Sparrow, Balla, \& Chicchetti, 1984 as cited in Franklin, Harris, \& Allen-Meares, 2006) and the Adaptive Behavior Assessment System, Second Edition (ABAS II) (Harrison \& Oakland, 2003). These adaptive behavior scales are developed for use with individuals when developmental delays or mental retardation are suspected. The Behavior Assessment System for Children, Second Edition (Reynolds \& Kamphaus, 2004) measures adaptive behaviors in individuals that do not necessarily have developmental delays or mental retardation; but may be experiencing behavioral or academic problems. The current body of literature focuses primarily on extinguishing maladaptive behaviors and does not provide an in-depth analysis of building adaptive skills for students identified as having problem behaviors.

\section{Rating Scales}

Rating scales allow objective assessment of behavior, and make it possible to obtain information about the occurrence of rare behaviors, and to capture information regarding the occurrence of rare behaviors that would likely not been seen in a time-sampling measure (Hart \& 
Lahey, 1999). Hart and Lahey (1999) found that generally speaking, rating scales (a) make use of standard instructions and response formats, (b) are designed to minimize subjective ratings, (c) allow the calculation of quantitative scores for each area of functioning, (d) have clinical cutoffs, and (e) are standardized on large samples. The authors found that although clinical interviews are an excellent source of information they do not provide the objective and systematic data collection that is present in rating scales. This method of collecting data allows for a possible comparison of a student to a relative normative group and/or to compare relevant data across differing points in time. Sajatovic and Ramirez (2003) found that most rating scales used in mental health services are ordinal scales, that is, the numbers represent labels of categories reflecting an increased order for the item being measured. Ordinal scales do lack the accuracy of interval scales, where the changes are constant and represent the same value. Sajatovic and Ramirez (2003) found that to achieve consistency with ordinal scales it is important to "have definitions and/or guidelines for the items/anchoring points in order to assign the proper numerical values and to minimize the personal bias of the rater" (p. 11). The Behavior Assessment System of Children, Second Edition (BASC-2) utilizes an ordinal scale for rating problematic and adaptive behaviors. The respondent answers questions on a scale of Never (N), Sometimes (S), Often (O), or Always (A).

The past two decades have seen a dramatic increase in the availability of rating scales and checklists designed for use in the assessment of emotional and behavioral functioning of children and adolescents (Hart \& Lahey, 1999). Scales used commonly in clinical and educational settings include the Conners' scales, the Achenbach Child Behavior Checklist, and the Behavior Assessment System for Children (Hart \& Lahey, 1999). The authors also found that there has been an increase in sophistication in the development of these behavioral rating scales, with 
more attention to issues of reliability and validity, as well as developmental considerations. As a result, there are a wider variety and greater number of, empirically sound instruments to choose from in clinical, educational, and research settings.

Rating scales provide objective data that is collected by individuals who may know the person being rated, or may observe the person for the first time that is being rated. Reliability and validity are important issues that are raised when dealing with rating scales. Reliability is the ability of the scale to convey consistent and reproducible information (Sajatovic \& Ramirez, 2003). Hart and Lahey (1999) found that there are several types of reliability that typically relate to rating scales: test-retest reliability, interrater reliability, and internal consistency. Test-retest reliability is the likelihood of obtaining the same score on two separate occasions. Interrater reliability is the likelihood that two different raters will arrive at the same score. Internal consistency is the extent to which items on the scale are measuring the same content. Many parent and teacher rating scales have high internal consistency and yield high test-retest reliability $(.80-.90)$ (Hart \& Lahey, 1999). According to the authors, interrater reliability is more difficult to determine because of a discrepancy that may exist due to a child's behavior varying across settings, and it may also be due to raters having differing response tendencies, or one of the raters may not be reliable. Overall, Hart and Lahey (1999) found that "most rating scales designed for adult informants are highly reliable" (p. 67).

Validity, according to Sajatovic and Ramirez (2003), is the degree to which the scale measures what it is supposed to measure. There are several types of validity that can be examined in relation to rating scales: construct validity, content validity, face validity, and criterion validity. According to Sajatovic and Ramirez (2003) most validation studies of rating scales will start with examining content validity. Content validity is the extent to which the scale 
items fully represent the content to be measured (Sajatovic \& Ramirez, 2003;Hart \& Lahey, 1999). The attributes of content validity, according to Sajatovic and Ramirez (2003) are more subjective than statistical, with clinical credibility of a measure inferred from expert comments and/or patients. The authors found that criterion validity is based much more on statistical analyses; this measures the extent to which scores on the measure predict scores on other relevant measures (Sajatovic \& Ramirez, 2003). Criterion validity may be either concurrent or predictive (Sajatovic \& Ramirez. 2003). Concurrent criterion validity refers to when the relevant criterion is measured at the same time, and predictive criterion validity refers to when the outcome criterion is measured at a later time (Sajatovic \& Ramirez, 2003).

Hart and Lahey (1999) found that several factors can assist in increasing the reliability and validity of rating scales; these include having clearly defined anchor points, the inclusion of more than two rating points, and raters with extensive experience with children and adolescents. The final consideration in choosing an appropriate behavior rating scale, according to Hart and Lahey (1999), is clinical utility. Clinical utility, according to the authors answers the following questions: (a) is the chosen scale one that is relevant to the questions to be addressed in the assessment, (b) is it acceptable to the rater, (c) does it provide adequate coverage, (d) is it brief enough to be practical, (e) is it sensitive to the treatment effects. Rating scales used early on in the treatment process can easily serve as springboards to providing appropriate and adequate treatment to an individual (Hart \& Lahey, 1999).

Weist et al. (2000) call for expanded school mental health services to take into consideration developmental and cultural factors. It also appears that behavioral rating scales also need to take into account relevant cultural factors when assessing a student's behavior. 
Behavior rating scales today are broadening their scope of clinical normative sampling to include a much more diverse range of cultural and socio-economic factors into their samples. Behavioral Assessment System for Children, Second Edition (BASC-2)

The BASC-2 (Behavior Assessment System for Children, Second Edition), which was recently released by AGS Publishing, is a multidimensional tool used by mental health and school professionals to collect data for a comprehensive assessment. The BASC-2, according to Reynolds and Kamphaus (2004), is a "multimethod, multidimensional system use to evaluate the behavior and self-perceptions of children and young adults aged 2 through 25 years" (p. 1). The wide acceptance of the original BASC (Reynolds \& Kamphaus, 1992), made the revisions minor in many areas. The BASC-2 is the newest revision of the original BASC and although new, the authors employed the guiding rubric over the original BASC of "if it ain't broke don't fix it" (Reynold \& Kamphaus, 2004, p. 2). Some improvements on the BASC-2 relevant to this study, although minor, include the following: new scale and item content, improved normative samples, new software and interpretive reports, and additional product offerings. The improved normative sample is the greatest improvement of the BASC-2. Standardization forms were collected in 257 cities in 40 U.S. states, including Hawaii (Reynolds \& Kamphaus, 2004).

The BASC-2 has multiple components that can be used to collect a variety of information regarding students. There is a Teacher Rating Scale (TRS), a Parent Rating Scale (PRS), and a Self-Report of Personality (SRP) that collect similar information from multiple informants. Reynolds and Kamphaus (2004) and Hart and Layhey (1999) found that different informants provide a clinician with different information, and therefore the TRS, PRS, and SRP all attempt to gain the most relevant information from the correct person, so that the rating scale can be completed in a sensible period of time. There is also a Student Observation System (SOS) that 
can be used by trained professionals to conduct structured observations of students for additional information (Reynolds \& Kamphaus, 2004). The BASC-2 includes a Structured Developmental History (SDH) that can be used in an interview format or completed by a parent, caregiver, or guardian to collect relevant developmental, health, and family information (Reynolds \& Kamphaus, 2004). All of these components are being used in various capacities throughout the state in Hawaii by the Department of Education.

This study was primarily concerned with the use of the Teacher Rating Scale (TRS) used within the Hawaii Department of Education. According to Reynolds and Kamphaus (2004) the TRS is designed to be completed by an adult who has had the opportunity to observe the child in school or a similar setting (i.e. preschool). This adult, according to the authors, can be a teacher, teacher's aide, or someone else in a similar role. Reynolds and Kamphaus (2004) find that it is preferable to have a teacher complete the TRS who observes the student in a fairly organized class setting, where there is seatwork, group work, or teacher student interaction. Multiple ratings from multiple raters can be useful is determining how the student interacts with various teachers in various classroom settings. Valid results, according to Reynolds and Kamphaus (2004) can be obtained when the rater has had "a month of daily contact or 6 to 8 weeks of several-days-a-week observation" (p. 19). There are three forms with items applicable to three age groups (Reynolds \& Kamphaus, 2002): Teacher Rating Scale - Preschool (TRS-P) ages 2 5, Teacher Rating Scale - Child (TRS-C) ages 6-11, and Teacher Rating Scale - Adolescent (TRS-A) ages $12-21$. The forms contain descriptors of behaviors that the respondents rate on a four-point scale of frequency ranging from Never to Almost Always. A teacher who is familiar with the scale can, according to Reynolds and Kamphaus (2004), complete the scale in 10 to 15 minutes. 


\section{BASC-2 Scales}

The conceptualization of the BASC-2 scales is derived from the original BASC scales and based on a comprehensive review of existing rating scales, a vision to include adaptive and maladaptive behaviors, and consultation with various clinicians and other professionals (Reynolds \& Kamphaus, 2004). In developing the BASC the authors surveyed a large sample of teachers and students and asked them to describe the five most obnoxious, disturbing, or disruptive behaviors and the five most positive behaviors exhibited by students in the classroom. The results were utilized as test items for the BASC.

The BASC-2 Teacher Rating Scale (TRS) is comprised of several Composite scores. The Composite scores, according to Reynolds and Kamphaus (2004), "represent behavior dimensions that are distinct but not independent; problem behaviors often occur in concert rather than individually" (pg. 65). The BASC-2 TRS contains Composite scores for the following Externalizing Problems, Internalizing Problems, School Problems, Behavioral Symptoms Index, and Adaptive Skills. See Figure 1 for a summary of Scales associated with each Composite. This study will examine all of the Composite scores with the exception of the Behavioral Symptoms Index (BSI). The BSI, according to Reynolds and Kamphaus (2004), measures overall behavior very similarly to a Global Assessment of Functioning (GAF) score.

The BASC-2 TRS contains Clinical and Adaptive Scales that are grouped into each Composite score. The Clinical Scales, according to Reynolds and Kamphaus (2004), measure maladaptive behaviors. High scores on these scales indicate negative or undesirable behaviors. T-Scores in the range of 60 to 69 are considered At-Risk, and T-Scores 70 and above are Clinically Significant. Adaptive Scales measure positive behaviors, but unlike the Clinical 
Scales, high scores represent positive or desirable behaviors. T-Scores in the range of 31 to 40 are considered At-Risk, and T-Scores lower than 30 are Clinically Significant.

The Clinical Scales are composed of four Composite scores and ten Scale scores. The Adaptive Scales are composed of one Composite score and five Scale scores. The Clinical Scale of Externalizing Problems consists of the Scale scores of Hyperactivity, Aggression, and Conduct Problems. The Clinical Scale of Internalizing Problems consists of the Scale score of Anxiety, Depression, and Somatization. The Clinical Scale of School Problems consists of the Scale score of Attention Problems and Learning Problems. The Adaptive Scale of Adaptive Skills consists of the Scale scores of Adaptability, Social Skills, Leadership, Study Skills, and Functional Communication. See Figure 2 for a definition of each Scale.

\section{Hawaii's School-Based Mental Health System}

Schools across the country are moving to a more integrated model of intervention, where all services are provided at the school. This provides a multidisciplinary team that can meet regularly and coordinate its efforts. These school-based teams are a combination of educational and mental health services all with the same goals of helping individual students excel in the educational setting and beyond.

Hawaii's Department of Education (HDOE) has adopted a very similar model to the one Pumariega and Vance (1999) discuss. Pumariega and Vance (1999) found that advanced models of mental health service provide several levels of services to include all students in the school, not just students labeled with a disability. The authors found that these programs included primary preventative services for all students, secondary prevention services for at-risk students, and formal mental health services to be delivered during the school day. HDOE provides mental health services to students by individuals who are employed by HDOE. This model of service 
delivery increases the emphasis found to be effective by Weist et al (2000). HDOE has taken the provision of mental health services, at most levels, out of the hands of the private agency providers and placed it back with HDOE, where it can potentially be controlled better and an emphasis can be placed back on the educational component. HDOE does still work collaboratively with other child serving agencies to provide higher levels of care for student who are in the most need of these services (i.e. residential treatment facilities, partial hospitalization programs). With increased emphasis on providing services that are adaptable and can meet the client's needs in the least restrictive setting, new ways of providing counseling services are being developed. HDOE has developed a Comprehensive Student Support System (CSSS) to provide primary preventative services for all students in the state, secondary preventative services for atrisk students, and formal mental health services or school-based counseling services provided within the school setting. These school-based counseling services are now becoming more widely used to meet the student's needs (Slade, 2003).

Although many states have not yet completely adopted this model, Hawaii is one state that has been mandated to adopt this model. In Hawaii the Felix consent decree was instituted after a District Court case of Jennifer Felix et al. v. Benjamin Cayetano, et al. (Civil No. 9300367). This case was instrumental in the institution of many school-based mental health services in the state of Hawaii. This court decision occurred after a group of parents', school-age children were not receiving appropriate mental health services, filed a class-action law suite against the state of Hawaii. These parents contend that their children did not receive the necessary mental health services for their children. Initially, this legal proceeding was directed at the Department of Health in Hawaii, who typically provides mental health services to children and adolescents. The Department of Health was unable to comply with the court's rulings, and 
eventually the focus shifted to the Hawaii Department of Education (HDOE). The United States District Court for the District of Hawaii found that, "the state of Hawaii violated the Individuals with Disabilities Education Act (IDEA) by failing to provide required services to children with disabilities" (Felix v. Cayetano, 1984. The court referenced the concept of IDEA where all children have available to them a free and appropriate education, regardless of their disability (U.S. Department of Education, 2001). An interesting aspect of this case that is of concern today is the HDOE was ultimately the only child serving agency held responsible for providing these services. Felix v. Cayetano serves as an example of the transition of responsibility for providing mental health services to the school system. This trend in transitioning responsibility to the school system makes examining Hawaii’s system of interest.

Traditionally in other parts of the country, mental health services are monitored and paid for by Medicaid or private insurance carriers, the Department of Health (DOH) will at times provide services through non-profit agencies. The oversight and implementation of traditional mental health services, even when they are provided in non-traditional settings, is done by individual insurance carriers or by Medicaid. This leaves a very fragmented system of care where communication breakdowns are common (Pumareiga \& Vance, 1999). Hawaii is the only state where all schools are part of a statewide system of education, headed by the Department of Education (Center for Mental Health in the Schools, 2000). Hawaii, according to Center for Mental Health in the Schools (2000), has approximately 185,000 students, 11,400 teachers, and 667 school level administrative positions. There are 251 public schools housed in seven districts. In 2000, according to Center for Mental Health in the Schools, Hawaii estimated that approximately $40 \%$ of the student population brought with them some form of educational 
disadvantage. HDOE has adapted a model to be utilized, particularly with students at higher levels of care that need to access services from multiple systems of services.

In the state of Hawaii, a group of children and adolescents in need of mental health services were not receiving the services needed for them to benefit from their educational program. A class action lawsuit against the state of Hawaii was filed by parents who were not receiving services their children needed. The state of Hawaii was not providing educationally necessary services to these children and adolescents. The state of Hawaii was forced to act and a District Court Judge enacted what is now known as the "Felix Consent Decree" in the state of Hawaii. The Felix Consent Decree in effect transferred responsibility for mental health services for children and adolescents from Hawaii's Department of Health (HDOH) to Hawaii's Department of Education (HDOE). This was a major shift of funding and resources that took place only after the Department of Health was unable to comply with minimum regulations regarding service delivery and availability to children and adolescents. Hawaii's DOH continues to provide more intensive services, such as inpatient hospitalization, residential treatment, and partial hospitalization, but Hawaii's DOE provide lower levels of mental health service. Essentially, DOH was not able to fill positions needed to provide the mental health services for children and adolescents in the state of Hawaii.

The Felix Consent Decree placed a mandated level of care to be in place at predetermined benchmark periods, and the court would then monitor the progress of the Department of Education. The Department of Education is required to monitor their students receiving services under the Felix Consent Decree. These students are typically the neediest students in the state that remain in the public school system, requiring the highest level of care from Hawaii's DOE. The Department of Education was forced to develop services for students throughout the state 
with more intensive needs. Hawaii's Department of Education had been accustomed to providing only school services for their students. The typical protocol was to make appropriate referrals when necessary for "out-sourcing” mental health concerns. The Department of Education had employed guidance counselors and District school psychologists but master's level positions were added at each complex but they were providing school services not mental health services. Now, the Department of Education was forced to provide or sub-contract virtually all mental health services. This includes outpatient therapy, psychiatric evaluations and follow-up appointments, psychological evaluations, etc. The Department of Education will be providing access to all of these services for all students in the state.

The system that Hawaii's Department of Education has implemented provides a combination of school services and mental health services working as a team to provide the best possible care to each student in need of services. This is an integrative concept and approach to treatment that provides students with various professionals interacting together to meet the students' needs. The Department of Education developed what is now known as the Comprehensive Student Support System (CSSS) that clearly defines the role of the school and how the school system intends to meet the needs of the students. Under the CSSS, the Department of Education provides an array of support services utilizing various resources at the school, complex, and state level in a system of five distinct levels of student support. There are approximately 7,000 students who access services through the Comprehensive Student Support System (CSSS). CSSS provides an array of student support services to ensure that the support provided and the delivery of those services corresponds to the severity, complexity, and frequency of individual student needs. Each level increases in intensity or specialization of services. 
The five levels of student support services are as follows (Adapted from The CSSS School Newsletter, October 2004):

Level 1 - Basic Support for All Students

Students have diverse needs that are addressed in the regular classroom. The key to successful student support is the classroom teacher who involves the student and family working together. This level does not require a formal meeting or written plan, but it does require commitment to understanding. Teachers and other school staff regularly provide students with support and guidance at school and assist families in addressing the student needs at home.

Level 2 - Informal Additional Support Through Collaboration

This is additional support beyond what the regular classroom teacher typically provides. There is more targeted collaboration with various other school personnel (e.g. counselor, administrator, department or grade level teachers). These activities are carried out in an informal, supportive manner.

Level 3 - Individualized School and Community Based Programs

These services encompass further assistance for specific groups or needs. These services are generally provided after the Student Support Team (SST) meeting. The SST meeting includes parents, family members, teachers and/or other school or community support staff. The team members develop an action plan that addresses the students' needs. Staff and families continue to be involved in monitoring progress.

Level 4 - Specialized Services from DOE and/or Other Agencies

When a student requires specialized assessment or assistance, a Student Support Team (SST) is convened to collaboratively meet the needs of the student. The SST develops an action plan that may include a referral for Section 504, special education, related services, 
community agency support services, mental health services, as well as Level 1, 2, and 3 services. At this level there are IDEA, Section 504, equity, and other legal requirements that must be complied with.

\section{Level 5 - Intensive and Multiple Agency Services}

When the needs of the student and family require intensive multiple agency involvement, the SST develops an action plan that integrates the resources and services of DOE and other agencies. Student placement may be off-campus therapeutic and/or educational programs. School continues to dialogue with the families and agencies to ensure a smooth transition.

These specific levels of care were developed in order to provide the students with specific services related to their individual needs. This system of care is relatively new in Hawaii and is developing as it grows. Determining if the services are effective is a key component in providing the best possible care for individual students as the system is intended to do.

Providing mental health services to Asian American and Pacific Islander populations in Hawaii provides a unique opportunity to conduct research in an area that is lacking in the literature today. Hawaii's Department of Education (HDOE) appears to be on the cutting edge of developing and implementing new services. HDOE has fostered Sue et al. (1991) strategies of cultural sensitivity, by having teachers, counseling staff, and other professionals in the schools who are of a diverse cultural and ethic background. HDOE actively recruits native Hawaiian Islanders, and is actively seeking out individuals of diverse cultures to fill positions within the Department of Education. Sue et al. (1991) cited the strategies for change to include training of personnel to work with culturally dissimilar clients, the employment of more bilingual and 
bicultural mental health workers, and the establishment of parallel services specifically designed for ethnic minority clients. HDOE is utilizing this concept to a large extent to assist in improving their educational programming.

\section{Statement of Problem}

Mental health services for children and adolescents have evolved and changed over the past century. The most recent development of these mental health services is located within the schools and utilizes school-based mental health personnel. Measuring the effectiveness of these services is difficult. According to Hart and Lahey (1999) rating scales allow objective assessment of behavior and for a possible comparison of a student to a relative norm group and to compare relevant data across different points in time. Measuring effectiveness of these services is further complicated by studying large minority populations.

Secondly, referrals are made for counseling services and the individual counselors are given the latitude to determine what type of counseling the student will receive (individual counseling, group counseling, or family education). There is no data compiled to assist the counseling provider in determining which type of counseling service is likely to give the student the most favorable outcome. In the past, the state of Hawaii was using the Achenbach Child Behavior Checklist (CBCL) to collect data on students receiving counseling as a related service. The state of Hawaii determined that the CBCL was much more of a clinical scale and possibly even a tool that searches for pathology in children, therefore it was decided to switch to the BASC-2, as it was geared more toward the educational setting and contained Adaptive scales. The BASC-2 also has a large normative sample which includes students from Hawaii. Minutes of counseling are determined by the IEP teams and are incorporated into IEPs for students. These minutes are logged by individual counseling providers as they are provided. 
The counseling minutes are recorded as individual counseling, group counseling, and family education. This information is not compared to any changes on the BASC-2 Teacher Rating Scale (TRS) at this time. Comparing this data from students' TRS over a one year time period may be helpful in determining if the interventions conducted were effective. The results of that analysis of data can be generalized to other students and used to better plan interventions in the future.

\section{Purpose of Proposed Study}

The purpose of the present study is to examine the effectiveness of school-based mental health services on adaptive behaviors of children and adolescents in the public schools in Hawaii. In particular, the education and mental health fields need to be informed of the most effective ways to provide school-based mental health services to children and adolescents. This study will gather baseline data from teachers regarding students who have been referred for counseling as a related service as part of their IEP. The data will be collected using the Behavior Assessment System for Children, Second Edition (BASC-2). The Teacher Rating Scale (TRS) of the BASC-2 will be used as the scale to collect the data on the selected students. The students will then receive counseling services from a counseling provider in the school setting. The counseling provider will log the number of minutes each student receives of each type of counseling service (individual, group, and family education). Follow up data will be collected from teachers in one year again using the Teacher Rating Scale of the BASC-2. The data collected on the Teacher Rating Scale of the BASC-2 will be examined at the baseline phase to determine if there is an elevated Composite Scale (Internalizing Problems, Externalizing Problems, School Problems, or Adaptive Functioning) of the BASC-2 TRS. The Department of 
Education in Hawaii has changed the scale used to collect data on students receiving counseling services to the BASC-2 after attempting to use the Achenbach Child Behavior Checklist.

The goal of this study is to examine the relationship between various counseling services (individual, group, and family education) provided in the school setting in relation to each student's BASC-2 TRS Composite Scale elevation. By examining this relationship school and mental health professionals will have the ability to more appropriately assist counseling providers in providing services to students in the school setting. The results will assist IEP teams and counseling providers in providing the most effective counseling service (individual, group and parent education) to students in the school setting.

\section{Limitations}

The author recognizes several substantial limitations to the proposed study. First, although the BASC-2 Teacher Rating Scale (TRS) can be used by different teachers and reliable results are attainable, it should be noted that different teachers will be collecting the Pre assessment and Post assessment TRS that are used for this study. This may create some difficulties in regard to interrater reliability. The Pre assessment data will be collected during January, February, or March of 2005 and the Post assessment data will be collected during January, February, or March of 2006. This adds an additional limitation of only having two data points to compare for the study. One way to counter balance the limited number of data points is by having a large sample to draw from. This study will be comprised of a fairly large sample of 275 students. Another limitation is the possibility that students may have multiple deficit areas. This will provide the author with additional data that may need to be examined in a different manner than originally proposed. Another limitation of this study is that only students referred in the first quarter (January, February, and March) of 2005 will be used in this study. This 
sample may not be a representative sample of the entire population of the students in Hawaii and generalizability of results may be limited. An additional limitation of this study is that there is no standardized method to determine which teacher the BASC-2 TRS should be collected from. Counseling providers utilize their judgment to collect as few as one BASC-2 TRS or as many as they feel necessary. There is no way to determine if the counseling provider has requested a BASC-2 TRS from an appropriate teacher. An additional limitation is the possibility that the counseling provider may not have accurately recorded the minutes of counseling service in their log. This study depends on accurate recordings of counseling minutes in the counseling provider logs. The results may be skewed if a large number of counseling providers do not accurately record their counseling minutes. The final limitation is in the final results and their generalizability to the general population. Hawaii presents large cultural diversity that is unparalleled anywhere else. This limitation may make applying these results to other populations difficult. The approach of providing counseling services within the context of the school may be very generalizable to other areas and diagnoses, although the results may vary in depending on cultural diversity in those areas.

\section{Definition of Key Terms}

Several terms that are used throughout this study need definitions for the reader to better understand the services and relating factors.

Counseling as a related service - This is a term that describes counseling services provided in the school setting by counseling providers. These are counseling services that are recommended by a student's IEP/Modification Plan (MP) team and are provided when it is believed that counseling will assist students in meeting their goals. These 
counseling services are recommended when it is thought that the student's problems are impacting his or her education.

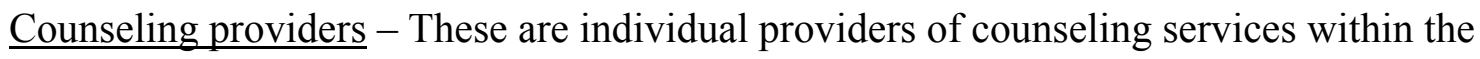
school setting. In Hawaii these counseling providers have a wide variety of education and experience. Counseling providers generally refer to the following:

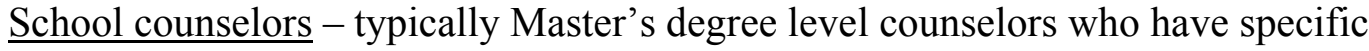
training in providing counseling services within the school setting.

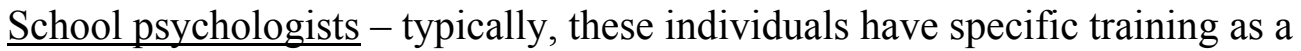
school psychologist with a Master's degree plus an additional thirty credits specifically in school psychology. There are also doctoral level school psychologists who have been trained at the Master's degree level and have returned for the additional doctoral degree usually in school psychology. The state of Hawaii does not certify or license school psychologists, but the title of school psychologist is protected and can only be used by those employed in a school setting. Licensing board allows school psychologists to practice psychology and call themselves a psychologist without becoming independently licensed but only for work they do in the Department of Education. Clinical psychologists - these are doctoral level trained psychologists who often have some school psychology experience, but this is not a requirement for this position. These clinical psychologists can be hired by the school districts and are given a two year period in which to get licensed as a psychologist within the state of Hawaii. 
$\underline{\text { Social workers }}$ - these are individuals who have a Master's degree in social work and are trained in social work interventions. These individuals generally have some experience working within a school setting and working with school age children and adolescents.

$\underline{\text { Behavioral Specialists }}$ - these are individuals who may hold a variety of different Master's degrees, including psychology, social work, or counseling or a closely related field. The Behavioral Specialists are individuals that have been hired under the Felix Consent Decree to provide not only traditional counseling services but also provide specific behavioral interventions. These individuals will have additional training, either in the form of formal graduate coursework or clinical trainings, in behavioral interventions. These individuals are included as counseling providers, and will often provide these services, but they will also provide additional behavioral interventions when necessary.

$\underline{\text { School-based mental health services }}$ - These are mental health services that are provided within the school setting. These services are provided within the school setting by either private agency personnel or by school staff.

Felix Consent Decree - This is a legal action imposed by the court system in the state of Hawaii. The Felix Decree, as it is often referred to, is a legal proceeding that was enacted after the Department of Health was unable to provide medically necessary services to children and adolescents. The state of Hawaii eventually moved the state level funds over to the Department of Education that is now responsible for providing virtually all mental health services to school-age students. The Department of Education reports to 
the court each quarter on the services that they have provided to all students across the state.

Comprehensive Student Support System (CSSS) - This is part of the system of care developed by the Department of Education in order to effectively service all students within the educational system, despite the intensity or severity of the student's problems or concerns. This system is also designed to provide all students within the school system the availability of services, even at a low intensity level.

Level 4 Services - This is the forth Level of service provided within the Comprehensive Student Support System (CSSS). Students may qualify for special education services, 504 Rehabilitation services, mental health services, or other community support services. Students at this level of care are at-risk for higher levels of services and interventions by the school are necessary.

Level 5 Services - This is the fifth and highest Level of service provided within the Comprehensive Student Support System (CSSS). At this level of care the student and family require a more intensive multiple agency approach and involvement. Students at this level have more severe and complex behavioral and/or emotional problems, which require more specialized programming including Therapeutic Classrooms, Enhanced Learning Centers, or off-campus placement. Behavioral Assessment System for Children, Second Edition (BASC-2) - This a multidimensional rating system used to evaluate a student's behaviors. The BASC-2 was published and released for use in 2004. There are multiple components to the BASC-2 system, including Teacher Rating Scale (TRS), Parent Rating Scale (PRS), Self-Report of Personality (SRP), Structured Developmental History (SDH), and a Student Observation 
System (SOS). These components will all be discussed in more detail throughout this study, but much of the study will focus on information gathered from the Teacher Rating Scale (TRS). The Teacher Rating Scale is a comprehensive measurement of both adaptive and problem behaviors, specific to the school setting. Teachers or others in similar roles as teachers (i.e. teacher aides) complete age appropriate forms that describe behaviors. The teacher rates the behavior of the student on a four-point scale Likert scale of frequency from Never to Almost Always. The TRS is scored using the BASC-2 Assist Plus software scoring program, which provides scale scores and composite scores relative to the student's problem areas and adaptive functioning areas. The software also identifies critical items that may be significant when answered in a particular manner. The BASC-2 software has a unique feature that allows the user to score the results, once entered into the computer, as compared to general population norms (either gender specific or combined sex norms), or specific clinical condition norm groups (all clinical conditions norms, ADHD norms, or Learning Disabled norms). Once the user has entered the data into the scoring software, the flexibility is there for the user to compare the scores to different normative groups, or alter the normative comparisons multiple times.

Internalizing Problems - These are problems or symptoms that are generally referred to as having an internal locus of control. For this study Internalizing Problems is a Composite Scale that is defined by the Teacher Rating Scale of the BASC-2. There are Primary Scales that make-up this Composite Scale; they are the Primary Scales of Anxiety, Depression, and Somatization. 
Externalizing Problems - These are problems or symptoms that have an external locus of control. For this study Externalizing Problems is a Composite Scale that is defined by the Teacher Rating Scale of the BASC-2. There are Primary Scales that make-up this Composite Scale; they are the Primary Scales of Hyperactivity, Aggression, and Conduct Problems.

$\underline{\text { School Problems }}$ - These are problems or symptoms that are related to academic or behavioral problems in the school setting, they are typically externalizing in nature, because they are most easily observed in the school setting. For this study, School Problems is a Composite Scale that is defined by the Teacher Rating Scale of the BASC2. There are Primary Scales that make-up this Composite Scale; they are the Primary Scales of Attention Problems, Learning Problems.

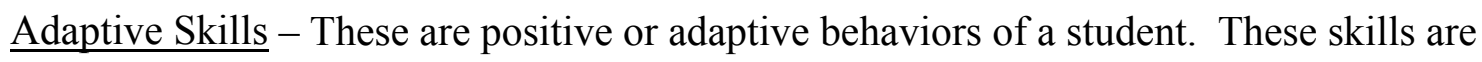
related to the school setting and are generally thought of by most as positive in nature. For this study Adaptive Skills is a Composite Scale that is defined by the Teacher Rating Scale of the BASC-2. There are Primary Scales that make-up this Composite Scale; they are the Primary Scales of Adaptability, Social Skills, Leadership, Study Skills, and Functional Communication.

\section{Research Questions}

This study of effectiveness of "counseling as a related service" in Hawaii's public schools as measured by the Behavioral Assessment System for Children, Second Edition's (BASC-2) purpose was to seek answer several Research Questions upon its completion. These Research Questions are accompanied by Research Hypotheses indicating expected effects. Null 
Hypotheses are not stated, but statistical tests will be used to determine rejection or non-rejection of such hypotheses.

\section{$\underline{\text { Research Question 1: }}$}

Do school-based mental health services with children and adolescents who receive Level 4 and Level 5 services who have externalizing problems change after interventions (individual counseling, group counseling, and/or family education)? Research Hypothesis 1 is that T Scores will decrease from Pre assessment to Post assessment for students who have externalizing problems and who receive individual counseling, group counseling, and/or family education services.

\section{$\underline{\text { Research Question 1a: }}$}

Do mental health services with children and adolescents who receive Level 4 and Level 5 services who have hyperactivity problems change after interventions (individual counseling, group counseling, and/or family education)? Research Hypothesis 1a is that T Scores will decrease from Pre assessment to Post assessment for students who have hyperactivity problems and who receive individual counseling, group counseling, and/or family education services. $\underline{\text { Research Question 1b: }}$

Do mental health services with children and adolescents who receive Level 4 and Level 5 services who have aggression problems change after interventions (individual counseling, group counseling, and/or family education)? Research Hypothesis $1 \mathrm{~b}$ is that T Scores will decrease from Pre assessment to Post assessment for students who have aggression problems and who receive individual counseling, group counseling, and/or family education services. 


\section{Research Question 1c:}

Do mental health services with children and adolescents who receive Level 4 and Level 5 services who have conduct problems change after interventions (individual counseling, group counseling, and/or family education)? Research Hypothesis 1c is that T Scores will decrease from Pre assessment to Post assessment for students who have conduct problems and who receive individual counseling, group counseling, and/or family education services.

\section{Research Question 2:}

Do mental health services with children and adolescents who receive Level 4 and Level 5 services who have internalizing problems change after interventions (individual counseling, group counseling, and/or family education)? Research Hypothesis 2 is that T Scores will decrease from Pre assessment to Post assessment for students who have internalizing problems and who receive individual counseling, group counseling, and/or family education services.

$\underline{\text { Research Question 2a: }}$

Do mental health services with children and adolescents who receive Level 4 and Level 5 services who have anxiety problems change after interventions (individual counseling, group counseling, and/or family education)? Research Hypothesis $2 \mathrm{a}$ is that $\mathrm{T}$ Scores will decrease from Pre assessment to Post assessment for students who have anxiety problems and who receive individual counseling, group counseling, and/or family education services.

\section{$\underline{\text { Research Question 2b: }}$}


Do mental health services with children and adolescents who receive Level 4 and Level 5 services who have depression problems change after interventions (individual counseling, group counseling, and/or family education)? Research Hypothesis $2 \mathrm{~b}$ is that $\mathrm{T}$ Scores will decrease from Pre assessment to Post assessment for students who have depression problems and who receive individual counseling, group counseling, and/or family education services. Research Question 2c:

Do mental health services with children and adolescents who receive Level 4 and Level 5 services who have somatization problems change after interventions (individual counseling, group counseling, and/or family education)? Research Hypothesis 1 is that T Scores will decrease from Pre assessment to Post assessment for students who have somatization problems and who receive individual counseling, group counseling, and/or family education services.

\section{$\underline{\text { Research Question 3: }}$}

Do mental health services with children and adolescents who receive Level 4 and Level 5 services who have school problems change after interventions (individual counseling, group counseling, and/or family education)? Research Hypothesis 3 is that T Scores will decrease from Pre assessment to Post assessment for students who have school problems and who receive individual counseling, group counseling, and/or family education services.

\section{$\underline{\text { Research Question 3a: }}$}

Do mental health services with children and adolescents who receive Level 4 and Level 5 services who have attention problems change after interventions 
(individual counseling, group counseling, and/or family education)? Research Hypothesis $3 \mathrm{a}$ is that $\mathrm{T}$ Scores will decrease from Pre assessment to Post assessment for students who have attention problems and who receive individual counseling, group counseling, and/or family education services.

\section{Research Question 3b:}

Do mental health services with children and adolescents who receive Level 4 and Level 5 services who have learning problems change after interventions (individual counseling, group counseling, and/or family education)? Research Hypothesis $3 \mathrm{~b}$ is that T Scores will decrease from Pre assessment to Post assessment for students who have learning problems and who receive individual counseling, group counseling, and/or family education services.

\section{$\underline{\text { Research Question 4: }}$}

Do mental health services with children and adolescents who receive Level 4 and Level 5 services who have adaptive skills deficits change after interventions (individual counseling, group counseling, and/or family education)? Research Hypothesis 4 is that $\mathrm{T}$ Scores will increase from Pre assessment to Post assessment for students who have adaptive skills deficits and who receive individual counseling, group counseling, and/or family education services.

\section{$\underline{\text { Research Question 4a: }}$}

Do mental health services with children and adolescents who receive Level 4 and Level 5 services who have adaptability deficits change after interventions (individual counseling, group counseling, and/or family education)? Research Hypothesis $4 \mathrm{a}$ is that $\mathrm{T}$ Scores will increase from Pre assessment to Post 
assessment for students who have adaptability deficits and who receive individual counseling, group counseling, and/or family education services.

$\underline{\text { Research Question 4b: }}$

Do mental health services with children and adolescents who receive Level 4 and Level 5 services who have social skills deficits change after interventions (individual counseling, group counseling, and/or family education)? Research Hypothesis $4 \mathrm{~b}$ is that $\mathrm{T}$ Scores will increase from Pre assessment to Post assessment for students who have social skills deficits and who receive individual counseling, group counseling, and/or family education services.

\section{$\underline{\text { Research Question 4c: }}$}

Do mental health services with children and adolescents who receive Level 4 and Level 5 services who have leadership deficits change after interventions (individual counseling, group counseling, and/or family education)? Research Hypothesis $4 \mathrm{c}$ is that $\mathrm{T}$ Scores will increase from Pre assessment to Post assessment for students who have leadership deficits and who receive individual counseling, group counseling, and/or family education services.

\section{$\underline{\text { Research Question 4d: }}$}

Do mental health services with children and adolescents who receive Level 4 and Level 5 services who have study skills deficits change after interventions (individual counseling, group counseling, and/or family education)? Research Hypothesis $4 \mathrm{~d}$ is that $\mathrm{T}$ Scores will increase from Pre assessment to Post assessment for students who have study skills deficits and who receive individual counseling, group counseling, and/or family education services. 


\section{Research Question 4e:}

Do mental health services with children and adolescents who receive Level 4 and Level 5 services who have functional communication deficits change after interventions (individual counseling, group counseling, and/or family education)? Research Hypothesis 4e is that T Scores will increase from Pre assessment to Post assessment for students who have functional communication deficits and who receive individual counseling, group counseling, and/or family education services.

\section{$\underline{\text { Research Question 5: }}$}

What type of school-based mental health service (individual counseling, group counseling, and/or family education) has the greatest impact on change in T-scores on children with externalizing problems who receive Level 4 and Level 5 services?

Research Hypothesis 5 is that T Scores will be impacted greatest from Pre assessment to Post assessment for students who have externalizing problems and receive individual counseling and family education.

\section{$\underline{\text { Research Question 5a: }}$}

What type of school-based mental health service (individual counseling, group counseling, and/or family education) has the greatest impact on change in Tscores on children with hyperactivity problems who receive Level 4 and Level 5 services? Research Hypothesis 5a is that $\mathrm{T}$ Scores will be impacted greatest from Pre assessment to Post assessment for students who have hyperactivity problems and receive individual counseling and family education.

\section{Research Question 5b:}


What type of school-based mental health service (individual counseling, group counseling, and/or family education) has the greatest impact on change in Tscores on children with aggression problems who receive Level 4 and Level 5 services? Research Hypothesis $5 \mathrm{~b}$ is that $\mathrm{T}$ Scores will be impacted greatest from Pre assessment to Post assessment for students who have aggression problems and receive individual counseling and family education.

\section{Research Question 5c:}

What type of school-based mental health service (individual counseling, group counseling, and/or family education) has the greatest impact on change in $\mathrm{T}$ scores on children with conduct problems who receive Level 4 and Level 5 services? Research Hypothesis $5 \mathrm{c}$ is that $\mathrm{T}$ Scores will be impacted greatest from Pre assessment to Post assessment for students who have conduct problems and receive individual counseling and family education.

\section{$\underline{\text { Research Question 6: }}$}

What type of school-based mental health service (individual counseling, group counseling, and/or family education) has the greatest impact on change in T-scores on children with internalizing problems who receive Level 4 and Level 5 services?

Research Hypothesis 6 is that T Scores will be impacted greatest from Pre assessment to Post assessment for students who have internalizing problems and receive individual counseling.

\section{Research Question 6a:}

What type of school-based mental health service (individual counseling, group counseling, and/or family education) has the greatest impact on change in T- 
scores on children with anxiety problems who receive Level 4 and Level 5 services? Research Hypothesis 6a is that T Scores will be impacted greatest from Pre assessment to Post assessment for students who have anxiety problems and receive individual counseling.

$\underline{\text { Research Question 6b: }}$

What type of school-based mental health service (individual counseling, group counseling, and/or family education) has the greatest impact on change in Tscores on children with depression problems who receive Level 4 and Level 5 services? Research Hypothesis $6 \mathrm{~b}$ is that $\mathrm{T}$ Scores will be impacted greatest from Pre assessment to Post assessment for students who have depression problems and receive individual counseling.

Research Question 6c:

What type of school-based mental health service (individual counseling, group counseling, and/or family education) has the greatest impact on change in Tscores on children with somatization problems who receive Level 4 and Level 5 services? Research Hypothesis $6 \mathrm{c}$ is that $\mathrm{T}$ Scores will be impacted greatest from Pre assessment to Post assessment for students who have somatization problems and receive individual counseling.

\section{$\underline{\text { Research Question 7: }}$}

What type of school-based mental health service (individual counseling, group counseling, and/or family education) has the greatest impact on change in T-scores on children with school-problems who receive Level 4 and Level 5 services? Research 
Hypothesis 7 is that T Scores will be impacted greatest from Pre assessment to Post assessment for students who have school-problems and receive group counseling. $\underline{\text { Research Question 7a: }}$

What type of school-based mental health service (individual counseling, group counseling, and/or family education) has the greatest impact on change in $\mathrm{T}$ scores on children with attention problems who receive Level 4 and Level 5 services? Research Hypothesis 7a is that $\mathrm{T}$ Scores will be impacted greatest from Pre assessment to Post assessment for students who have attention problems and receive group counseling.

$\underline{\text { Research Question 7b: }}$

What type of school-based mental health service (individual counseling, group counseling, and/or family education) has the greatest impact on change in Tscores on children with learning problems who receive Level 4 and Level 5 services? Research Hypothesis 5 is that T Scores will be impacted greatest from Pre assessment to Post assessment for students who have learning problems and receive group counseling.

\section{Research Question 8:}

What type of school-based mental health service (individual counseling, group counseling, and/or family education) has the greatest impact on change in T-scores on children with adaptive skills deficits who receive Level 4 and Level 5 services? Research Hypothesis 8 is that T Scores will be impacted greatest from Pre assessment to Post assessment for students who have adaptive skills deficits and receive individual counseling and group counseling. 
Research Question 8a:

What type of school-based mental health service (individual counseling, group counseling, and/or family education) has the greatest impact on change in $\mathrm{T}$ scores on children with adaptability deficits who receive Level 4 and Level 5 services? Research Hypothesis $8 \mathrm{a}$ is that $\mathrm{T}$ Scores will be impacted greatest from Pre assessment to Post assessment for students who have adaptability deficits and receive individual counseling and group counseling.

$\underline{\text { Research Question 8b: }}$

What type of school-based mental health service (individual counseling, group counseling, and/or family education) has the greatest impact on change in $\mathrm{T}$ scores on children with social skills deficits who receive Level 4 and Level 5 services? Research Hypothesis $8 \mathrm{~b}$ is that $\mathrm{T}$ Scores will be impacted greatest from Pre assessment to Post assessment for students who have social skills deficits and receive individual counseling and group counseling.

$\underline{\text { Research Question 8c: }}$

What type of school-based mental health service (individual counseling, group counseling, and/or family education) has the greatest impact on change in $\mathrm{T}$ scores on children with leadership deficits who receive Level 4 and Level 5 services? Research Hypothesis $8 \mathrm{c}$ is that $\mathrm{T}$ Scores will be impacted greatest from Pre assessment to Post assessment for students who have leadership deficits and receive individual counseling and group counseling.

\section{$\underline{\text { Research Question 8d: }}$}


What type of school-based mental health service (individual counseling, group counseling, and/or family education) has the greatest impact on change in Tscores on children with study skills deficits who receive Level 4 and Level 5 services? Research Hypothesis $8 \mathrm{~d}$ is that $\mathrm{T}$ Scores will be impacted greatest from Pre assessment to Post assessment for students who have study skills deficits and receive individual counseling and group counseling.

\section{$\underline{\text { Research Question 8e: }}$}

What type of school-based mental health service (individual counseling, group counseling, and/or family education) has the greatest impact on change in $\mathrm{T}$ scores on children with functional communication deficits who receive Level 4 and Level 5 services? Research Hypothesis $8 \mathrm{e}$ is that $\mathrm{T}$ Scores will be impacted greatest from Pre assessment to Post assessment for students who have functional communication deficits and receive individual counseling and group counseling. 


\section{CHAPTER II}

\section{METHOD}

\section{Introduction}

The purpose of this study was to examine the effectiveness of school-based mental health services. Both education and mental health fields need to be informed of the most effective ways to provide mental health services to children and adolescents within the school setting. This study was concerned with the relationship between students' T-Score elevations on the BASC-2 Teacher Rating Scale and types of counseling services (individual counseling, group counseling, and/or family education) received by school-based mental heath providers. This study's goal was to find a relationship between these variables in order to better assist counseling providers and IEP teams in developing specific programs for students in the school setting.

Rating scales provide objective assessment of behavior and the ability to then compare that data to selected normative groups (Hart \& Lahey, 1999; Reynolds \& Kamphaus, 2004). Studying ratings of child and adolescent behaviors in the school setting causes the following pragmatic problems: (a) which student populations to study, (b) is there a representative student sample within that population, and (c) how to gain access to that student sample. The goal of this study was to use a sample of students in a school system where the implementation of school-based mental health services was occurring. Locating a school system that was already utilizing school-based mental health services was important, due to the current findings in the literature regarding the future development of mental health services for children and adolescents being provided in the school setting. The Hawaii Department of Education (HDOE) is utilizing a school-based mental health service program currently. This program provides unique opportunities for research and investigation. The cultural diversity present in Hawaii, presents 
additional opportunities to examine effectiveness of not only school-based mental health services, but also of their use with culturally divergent populations. Using the BASC-2 rating scale allowed the author to gather objective information from teachers regarding students' behaviors. The use of the BASC-2 TRS allowed the author to examine a broader range of behaviors, including adaptive behaviors, and compare them to a combined sex normative sample of the general population. Finally, the use of minutes of counseling service (individual counseling, group counseling, and/or family education) allowed the author to examine possible correlations between services and BASC-2 T-Scores.

\section{Participants}

Data for this study was drawn from an existing database provided by Hawaii's Department of Education. The data was compiled by Hawaii's Department of Education staff and examined for completeness and timeliness. This study used 346 students from an existing database provided by HDOE to the principle investigator. The students were between the ages of 6 years of age and 18 years of age. Child and Adolescent versions of the BASC-2 scales were collected and used as data for the current study; these versions both contain 139 items that are comparable and can be used to examine change in behavior (Reynolds \& Kamphaus, 2004). HDOE staff coded and sanitized the data, removing all identifying information of participants. The participants for this study were selected based on the following criteria: (1) students were enrolled in Hawaii's public school system (students in approved private placements, those being home schooled, or not in the public school system were excluded), (2) students were receiving Level 4 or Level 5 services through the Comprehensive Student Support System (CSSS), (3) students were referred by the IEP team to receive "counseling as a related service" as part of the students IEP, (4) the students were referred to receive "counseling services" during the first 
quarter (January 3, through March 31) of 2005, and (5) valid BASC-2 Teacher Rating Scales were collected during the first quarter of the year to be used as baseline data. Only one BASC-2 TRS will be used per student per data collection period (January through March 2005 and January through March 2006). If more than one BASC-2 TRS is collected for a student only the first one collected will be utilized in this study. Students who did not meet all of the above criteria were excluded from the study. For example, any student who had been receiving ongoing counseling services prior to the first quarter would be excluded from this study. Likewise, students who receive "counseling services" at a lower level (i.e. Level 3) in the CSSS system will also be excluded from this study. Students who have invalid BASC-2 TRS scales will be dropped from the study. Invalid scales are relevant data, but not to be included in this study. Therefore, invalid TRS data will not be used in this study, as it will not be provided to the author for review.

The Hawaii Department of Education have all BASC-2 data reported by District School Psychologists on a quarterly basis. BASC-2 TRS will be collected from all students in the state of Hawaii who are receiving "counseling as a related service" as part of their IEP when they are also receiving Level 4 or Level 5 services as part of CSSS. The BASC-2 data is being collected as part of the Superintendent's initiative to track and report on students as part of the Felix Consent Decree (see Appendix A). Only students who are being referred during the first quarter of 2005 will be used in this study.

\section{Procedures}

Individual counseling providers are responsible for collecting BASC-2 data for students on their caseloads, by distributing the appropriate BASC-2 forms to individual teachers during the initial referral time from the IEP team. As part of the Hawaii Department of Education's 
program described by the Superintendent's memo (see Appendix A), at the initial referral time for "counseling as a related service" the counseling provider collects the Teacher Rating Scale (TRS), Parent Rating Scale (PRS), Self-Report of Personality (SRP), and the Structured Developmental History (SDH). This study will focus only on the data collected on the TRS. The reason for this distinction is that Reynolds and Kamphaus (2004) state that different information is best collected from various sources, so the questions on each of the rating scales (TRS, PRS, SRP, and SDH) seek to find out different information that can best be found from that source (i.e. teacher, parent, or student).

The initial TRS data serves as baseline data for students and for this study. Individual counseling providers have attended various workshops and in-service trainings through the Hawaii Department of Education regarding how to collect appropriate BASC-2 data. Counseling providers are required to collect the BASC-2 data from one teacher. The individual counseling provider does have the latitude and ability to use their judgment to determine which teacher to collect the BASC-2 TRS from. During trainings, which this investigator was a part of, held at schools throughout the state of Hawaii in December 2004, January 2005, and February 2005, individual counseling providers were provided with insight into various ways to determine which teacher to collect BASC-2 data from. Counseling providers were informed that if a student has trouble with only one teacher, collecting a BASC-2 TRS from that teacher may only provide insight to the glaring difficulties that student may have in that classroom, and may not provide any insight to adaptive functioning or strengths of the student. Likewise, collecting a TRS from a teacher where the student has no problems (i.e. art class) will not be nearly as helpful in assisting in the assessment and identification of problem behaviors. Hawaii's Department of 
Education has placed trust in their counseling providers to make good judgments in choosing who completes the TRS.

The TRS is collected and scored and placed into a database of BASC-2 TRS reports. This data was examined by HDOE for completeness and timeliness. The data was then coded and provided to the principle investigator of this study for examination. All identifying information was excluded from the files provided to the author.

The second TRS was completed at the one-year time from referral. The counseling provider again located the appropriate teacher and request that they complete the TRS. This was done during the first quarter (January, February, and March) of 2006. The data was again placed into a database of BASC-2 TRS reports. This data was again examined by HDOE for completeness and timeliness. All identifying information was excluded from the files provided to the principle investigator. The data collected by the counseling provider was and is maintained by the Hawaii Department of Education (HDOE). The data was coded and all identifiers were removed by HDOE prior to the data being provided to the principle investigator for examination and data analysis. The data was provided to the principle investigator in the form of electronic data logs on a high-density data key that is password protected.

\section{Counseling Services}

Counseling services provided to students in the public school system in Hawaii, vary widely. Counseling providers utilize a wide array of clinical interventions and techniques to provide students in Hawaii with counseling services. Counseling providers have a wide array of trainings and experiences to draw from when providing counseling services to students. Counseling providers are provided with on-going in-service trainings to improve the quality of interventions provided to the students in Hawaii's public school system. The counseling 
providers are encouraged to utilize the most current data and implement evidenced-based treatments to the students, although this is loosely monitored or imposed. Typically counseling providers have latitude to implement techniques and strategies that they are familiar and comfortable with to meet the needs of the students. For the students who are receiving Level 4 and Level 5 services the counseling providers are required to log the counseling minutes prescribed on the student's IEP. The counseling providers are required to classify these counseling services that are provided into one of three categories: individual counseling, group counseling, and family education. The definition of each of these services is an implied definition and not one that is readily available to counseling providers for review.

Individual counseling is commonly known as a one-to-one intervention between a counseling provider and a student. Individual counseling is recorded by the counseling provider at a time of contact with the student. Individual counseling may refer to traditional individual counseling sessions, but it may also refer to times when a counseling provider engages in a less structured conversation with a student (i.e. a counseling provider having a conversation with a student between classes or at lunch). Individual counseling is occurring when a counseling provider is meeting with a student directly to benefit that student by providing some direct intervention aimed at meeting goals of the student's IEP. Individual interventions can be to address a wide variety of issues for students, including, but not limited to, depression, anxiety, school problems, learning problems, coping skills development, anger or aggressive problems, or social skills deficits. Individual counseling can also be used to assist in building adaptive or prosocial skills with students.

Group counseling is commonly known as group interventions with the student and other students who are targeted for direct service. Typically, group interventions are recognized when 
a counseling provider is providing direct intervention to a group (small or large) of students who are seeking intervention for addressing some needs in a group treatment modality. Group counseling interventions are occurring when a student in being provided with a direct counseling intervention to address a specific goal on the child's IEP. Group counseling interventions can be for a wide array of problems, including, but not limited to, grief groups, study skills groups, divorce groups, and social skills groups.

Family education is the final category of counseling service provided by the counseling providers at the Department of Education in Hawaii's public schools. This category is the proverbial catch-all category for counseling providers. Family education can refer to the widest array of counseling services, and often implies that the services were consultative or collateral in nature, and not providing a direct service to the student. Family education can imply that the counseling provider did meet with the family to address some issue of relevance to the student, but it may also imply that the counseling provider met with a teacher or someone else relevant to the student, to address issues of relevance about the student. These services may include, but are not limited to, IEP meetings, student observations, teacher meetings, collection of student data, parent meetings, family meetings, or other indirect time a counseling provider spends working toward addressing specific goals on the students IEP.

\section{Counseling Providers}

Counseling providers vary widely between buildings and across islands. All counseling providers recording data for Level 4 and Level 5 students are Hawaii Department of Education employees. HDOE does at times utilize subcontractors to provide counseling services to students within the state. These subcontractors are typically used for specific counseling interventions and often times are used for out-of-district placements, such as partial 
hospitalization programs or residential programs. All of the counseling providers who log the counseling minutes for the students in this study are employees of HDOE. Counseling providers include HDOE staff in a variety of job duties including clinical psychologists, school psychologists, school counselors, school social workers. The collective backgrounds of these counseling providers are extremely diverse, as some are native to the islands of Hawaii and others are from across the planet. Many counseling providers have moved to Hawaii and have a wide breadth of clinical and educational experiences. Data on the diversity of counseling providers was not available, as it is not kept in a central database for review.

The education level of counseling providers also varies widely between buildings and across islands. Some counseling providers are doctoral level, others are masters prepared, and some were grandfathered into positions as counseling providers at the bachelor's level. Some of the counseling providers are licensed through state licensing boards as clinical psychologists, counselors, and social workers; others are licensed or certified through the Hawaii Department of Education as school psychologists, school counselors, and school social workers. Training of counseling providers varies widely, with some trained within the state of Hawaii and others from around the country. Currently counseling providers are hired at the masters and doctoral level only. Counseling providers hired now, typically possess a master's degree in counseling, psychology, educational psychology, social work, or some other closely related field, or a doctoral degree in counseling, clinical psychology, school psychology or another closely related field. DOE also now requires license or certification to retain employment. Many doctoral level counseling providers must be dually licensed and certified by the state licensing board and the department of education. Master's level counseling providers are typically certified through the department of education, but may also be licensed by the state licensing board. These minimum 
qualifications have raised the expectations for the counseling providers, in an attempt to improve the counseling service provided by HDOE. There are some counseling providers that do not meet these minimum criteria, as they were hired prior to any formal policy and are bachelor's level counseling providers. These providers were grandfathered into counseling positions, as they were hired prior to minimum standards were enforced. Data on diversity of counseling providers' education level was not available, as it is not kept in a central database for review. Instrument

The BASC-2 Teacher Rating Scale (TRS) will be utilized to collect data for this study. The normative sample used for the BASC-2 is collected from a large and representative sample of children across the United States. The sample, according to Reynolds and Kamphaus (2004), was designed to resemble the population with respect to sex, socioeconomic status (as indicated by parental education), race/ethnicity, geographic region, and classification in special education or gifted programs. The standardization of the BASC-2, according to Reynolds and Kamphaus (2004), took place from August 2002 through May 2004. The authors report that the General norm samples included a total of more than 13,000 children ages 2 through 18 years of age. Over 375 sites in 257 cities and 40 states were included in the collection of normative sampling. The authors utilized children from various settings, including public schools, private schools, mental health clinics, hospitals, and preschool and day care settings. Reynolds and Kamphaus (2004) report that the sample sizes for each norm group are very large and correlate to actual population percentages in the United States.

The BASC-2 has some unique features that allow the user to choose various normative groups for comparison. The BASC-2 allows the user to choose a broader category of either General norm sample or Clinical norm sample and also combined-sex or separate-sex norms for 
each broad category norm sample. The Clinical norm samples are subdivided into additional diagnostic categories including All Clinical Conditions (which combines a large sample of clinical conditions), Learning Disability, and Attention/Deficit- Hyperactivity Disorder. Reynolds and Kamphaus (phone conference April 2, 2005) recommend using the General combined sex norms for most scoring and interpretation. The user does have the availability to return to the software and score the results using other norm samples if they desire.

The Teacher Rating Scale (TRS) of the BASC-2 include Validity Indexes when using the computer software for scoring referred to as F Index, Consistency Index, and Response Pattern Index. The F Index is a score of the number of times the respondent gave a very negative behavior rating. These high scores can occur when a respondent is attempting to present problems worse than they actually are, however Reynolds and Kamphaus (2004) realize that negative responses can also be indicative of a child's actual behavioral concerns. The F Index was constructed on items that were rarely chosen during the development of the scale. See Table 1 in Appendix B for F Index Raw Score Frequency Summary. The Consistency Index identifies responses that are typically answered similarly that were answered differently by the respondent. The Consistency Index is very similar to a measure of random responding. The Response Pattern Index, according to Reynolds and Kamphaus (2004) is designed to identify a pattern of responses given by a respondent (i.e. answering $\mathrm{N}$ for all responses, preservative responses, or answering in a pattern of $\mathrm{N}, \mathrm{S}, \mathrm{O}, \mathrm{A}$, repetitively). The BASC-2 software will generate a raw score and an interpretive score of Acceptable, Caution, or Extreme Caution for all validity indexes on the TRS.

Reliability refers to the dependability, accuracy, or reproducibility of test scores (Reynolds \& Kamphaus, 2004; Hart \& Lahey, 1999). Reliability can be analyzed through 
internal consistency, test-retest reliability, and interrater reliability. Internal consistency, according to Hart and Lahey (1999) is the extent to which items on the scale are measuring the same content.

For the General norm samples, composite score reliabilities of the BASC-2 are very high: in the middle .90 s for composite score of Externalizing Problems, in the low to middle .90s for School Problems and Adaptive Skills composites, and in the high .80s to low .90s for Internalizing Problems composites. Reliabilities for individual scares are also high, according to Reynolds and Kamphaus (2004). Hyperactivity, Aggression, Conduct Problems, Attention Problems, and Learning Problems are reliable at the high .80s to low .90s. Social Skills, Leadership, Study Skills, and Functional Communication all have reliabilities in the .80s to low .90 s. Adaptability is also has a reliability in the .80 s to low .90 s except for the ages 2 through 3 , where the reliability is slightly lower at .76 to .82 . Reliability of Anxiety, Depression, and Somatization are somewhat lower that the others at .70s to the upper $.80 \mathrm{~s}$.

Test-retest reliability, according to Hart and Lahey (1999), is the likelihood of obtaining the same score on two separate occasions. Generally the test-retest reliabilities for the composite scales are in the middle .80 s to the low $.90 \mathrm{~s}$. For the individual scale scores the test-retest reliabilities are generally high. The median test-retest reliabilities are .82 for preschool, .86 for child, and .81 for adolescent.

Interrater reliability is the agreement of scores obtained from different raters who provided ratings at about the same point in time of the same student (Hart \& Lahey, 1999). According to Hart and Lahey (1999), interrater reliability is the most difficult to determine in behavior rating scales. Reynolds and Kamphaus (2004) found that at least three factors can cause the relationship between two teachers rating to be different than one teacher's ratings at 
two points in time. First, teachers have different interpretations of items. Secondly, they may perceive the intensity of the behaviors differently. Finally, the students' behavior may differ in various settings or in the presence of different teachers. Because of these limitations, the interrater reliability correlations are lower than other measures of reliability. The median interrater reliability for the composite scales are .65 for preschool, .56 for child, and .53 for adolescent. The interrater reliability across scale scores varies widely from .19 to .82 .

Validity of the BASC-2 is determined by examining the correlation between the BASC-2 and other behavior rating scales. The BASC-2 was compared to the Achenbach System of Empirically Based Assessment Caregiver-Teacher Report Form (ASEBA). Correlations between clinical scores overall range from .78 to .85, and correlations between the Externalizing Problems range from .75 to .85 . Internalizing problems correlations were more variable ranging from .64 to .80 . Externalizing problems are more closely correlated across instruments than internalizing problems.

The BASC-2 provides the user with clinical scales and adaptive scales for interpretation. The clinical scales measure maladaptive behaviors of students (Reynolds \& Kamphaus, 2004). The adaptive scales measure positive behaviors of students (Reynolds \& Kamphaus, 2004). There are a total of 14 clinical scales and 6 adaptive scales. The BASC- 2 also generates composite scores and scale scores. There are a total of 5 composite scores and 15 scale scores. Tables 2 provides a breakdown of composite scores and scale scores. Composite scores are helpful in drawing broad conclusions and summarizing performance regarding various adaptive and maladaptive behaviors (Reynolds \& Kamphaus, 2004). The authors based the composite scores on a representative dimension of behavior, realizing the problem behaviors often occur in concert rather than independently. Reynolds and Kamphaus (2004) state that, "individual items 
are narrow samples of behavior and therefore are unreliable indicators of broad behavior dimensions" (pg 67). A review of individual item responses may be necessary to understand specific reasons why an individual scale is elevated or depressed.

Reynolds and Kamphaus (2004) provide the user with a Systematic Approach to Interpretation of the BASC-2. The authors recommend that a careful review of validity scales is conducted prior to any interpretation. The authors recommend a simple process of interpreting the composite score scales first, then moving to the scale scores, and finally to individual items. For clinical scales the user should examine each composite score and determine which scores are in the Clinically Significant range $(\mathrm{T}=70+)$ and also scores in the At-Risk range $(\mathrm{T}=60-69)$. Reynolds and Kamphaus (2004) recommend that the best time to intervene with a child is when they are in the at-risk range of clinical significance. The use should also examine Adaptive Skills composite scores. The scores for adaptive functioning that are low are considered clinically significant. For Adaptive Skills the Clinically Significant range ( $\mathrm{T}=30$ and below) and At-Risk range $(\mathrm{T}=31$ to 40$)$ are opposites of the Clinical Composite scales. The user should examine scale scores in much the same manner, utilizing individual item interpretation as necessary to explain elevated or depressed scores.

Research Design and Analysis

The primary research question compared differences in pre-assessment time and post assessment time for a variety of variables. For this comparison the variables of composite TScores of Externalizing Problems, Internalizing Problems, School Problems, and Adaptive Skills were examined at pre-assessment time and at post-assessment time. To protect against "experiment-wise error-rate" and to allow subsequent comparisons to continue to be tested at a $p$ $<.05$ statistical significance, an initial Multivariate Analysis of Variance (MANOVA) was 
computed. When the initial MANOVA yields statistical significance, follow-up Analyses of Variance (ANOVAs) were computed. As illustrated in Table 3 in Appendix B the initial MANOVA for the overall research question will involve a within-subjects independent variable, Assessments Time (pre-assessment verses post-assessment). The dependant variables were TScores for Externalizing Problems, Internalizing Problems, School Problems, and Adaptive Skills.

Following the initial MANOVA on the four Composite scores as dependent variables, a MANOVA was calculated across the Scale scores for each research question. When the MANOVA yields a significant finding, the strategy calls for following up with ANOVA analyses. As illustrated in Table 3 in Appendix B the MANOVA for all four of these MANOVAs will involve a within-subjects independent variable, Assessment Time (preassessment versus post-assessment). For Research Question 1 the dependent variables will be TScores for Hyperactivity, Aggression, and Conduct Problems. For Research Question 2 the dependent variables will be T-Scores for Anxiety, Depression, and Somatization. For Research Question 3 the dependent variables will be T-Scores for Attention Problems and Learning Problems. For Research Question 4 the dependent variables will be T-Scores for Adaptability, Social Skills, Leadership, Study Skills, and Functional Communication.

To attempt to answer Research Question 5, a series of Multiple Linear Regression Analyses were computed, followed by Pearson Correlations for each relevant comparison. The variables for the Multiple Linear Regression Analyses are summarized in Table 4 in Appendix B.

As illustrated in Table 4 in Appendix B, a regression analysis was performed for each Scale Score change in $\mathrm{T}$ as the Criterion Variable. Thus, for Research Questions 5, 6, 7, and 8 the Criterion Variables will be Hyperactivity, Aggression, and Conduct Problems for Research 
Question 5; Anxiety, Depression, and Somatization for Research Question 6; Attention Problems and Learning Problems for Research Question 7; and Adaptability, Social Skills, Leadership, Study Skills, and Functional Communication for Research Question 8. As may be noted in Table 4 in Appendix B, the Predictor Variables in each of these regression analyses will be minutes of counseling service (individual counseling, group counseling, and family education).

\section{Power Analysis}

In the present investigation, a .05 alpha level was used, and beta was calculated at .20 (or four times alpha). This enabled the researcher to establish a desired level by setting the Type II error at four times the rate of making a Type I error (Keppel, 1991). A power estimation chart indicates that if power is set at .80 and a "medium" size effect is expected from the treatments, that an appropriate sample size would be $\mathrm{N}=44$, if the statistics are to be tested at the $p<.05$ level of significance (Keppel, 1991). A “medium” effect size was chosen to decrease the probability of Type I errors. 


\section{CHAPTER III}

\section{RESULTS}

The intent of this investigation was to test the effectiveness of school-based mental health services in Hawaii's public school system. The research questions and hypotheses are addressed through the analysis procedure and findings described. Where appropriate, an overall Multivariate Analysis of Variance (MANOVA) was computed to include multiple research question components. These inclusive analyses reduce the number of initial tests at the $p<.05$ level of statistical significance. Such reduction in the number of $p<.05$ statistical tests reduces the "experiment-wise error rate." Thus, the number of "significant" results that could occur by chance is minimized.

An overall MANOVA was computed to include Research Questions 1, 2, 3, and 4. In this MANOVA, the independent variable was Assessment Time (Pre-assessment versus Postassessment), and the dependent variables were Externalizing Problems, Internalizing Problems, School Problems, and Adaptive Skills. This analysis yielded a significant finding, $F(4,342)=$ $5.41, p<.01$, indicating the need to conduct the four component Analyses of Variance.

\section{Research Question 1}

Do school-based mental health services with children and adolescents who receive Level 4 and Level 5 services who have Externalizing Problems change after interventions (individual counseling, group counseling, and/or family education)? Research Hypothesis 1 is that T Scores will decrease from Pre-assessment to Post-assessment for students who have Externalizing Problems and who receive individual counseling, group counseling, and/or family education services. 
To test this hypothesis, a one-way, within-subjects (repeated-measures) Analysis of Variance was computed in which the independent variable was Assessment Time (Preassessment versus Post-assessment), and the dependent variable was Externalizing Problems. This analysis yielded $F(1,345)=20.35, p<.01$, indicating a statistically significant difference between the Pre-assessment and Post-assessment of Externalizing Problems. The mean for Preassessment (59.22) was significantly higher than the mean for the Post-assessment (56.50) of Externalizing Problems.

An overall MANOVA was computed to include Research Question 1a, 1b, and 1c. In this MANOVA, the independent variable was Assessment Time (Pre-assessment versus Postassessment, and the dependent variables were Hyperactivity Problems, Aggression Problems, and Conduct Problems. This analysis yielded a significant finding, $F(3,343)=7.08, p<.01$, indicating the need to conduct the three component Analyses of Variance.

Research Question 1a. Do mental health services with children and adolescents who receive Level 4 and Level 5 services who have Hyperactivity Problems change after interventions (individual counseling, group counseling, and/or family education)? Research Hypothesis 1a is that T Scores will decrease from Pre-assessment to Post-assessment for students who have Hyperactivity Problems and who receive individual counseling, group counseling, and/or family education services.

This hypothesis was tested by computing a one-way, within-subjects (repeated-measures) Analysis of Variance in which the independent variable was Assessment Time (Pre-assessment versus Post-assessment), and the dependent variable was Hyperactivity Problems. This analysis yielded $F(1,345)=17.08, p<.01$, indicating a statistically significant difference between the Pre-assessment and Post-assessment of Hyperactivity Problems. The mean for Pre-assessment 
(59.03) was significantly higher than the mean for Post-assessment (56.55) of Hyperactivity Problems.

Research Question 1b. Do mental health services with children and adolescents who receive Level 4 and Level 5 services who have Aggression Problems change after interventions (individual counseling, group counseling, and/or family education)? Research Hypothesis $1 \mathrm{~b}$ is that T Scores will decrease from Pre-assessment to Post-assessment for students who have Aggression Problems and who receive individual counseling, group counseling, and/or family education services.

This hypothesis was tested by computing a one-way, within-subjects (repeated-measures) Analysis of Variance in which the independent variable was Assessment Time (Pre-assessment versus Post-assessment), and the dependent variable was Aggression Problems. This analysis yielded $F(1,345)=18.77, p<.01$, indicating a statistically significant difference between the Pre-assessment and Post-assessment of Aggression Problems. The mean for Pre-assessment (59.35) was significantly higher than the mean for Post-assessment (56.43) of Aggression Problems.

Research Question 1c. Do mental health services with children and adolescents who receive Level 4 and Level 5 services who have Conduct Problems change after interventions (individual counseling, group counseling, and/or family education)? Research Hypothesis 1c is that T Scores will decrease from Pre-assessment to Post-assessment for students who have Conduct Problems and who receive individual counseling, group counseling, and/or family education services.

This hypothesis was tested by computing a one-way, within-subjects (repeated-measures) Analysis of Variance in which the independent variable was Assessment Time (Pre-assessment 
versus Post-assessment), and the dependent variable was Conduct Problems. This analysis yielded $F(1,345)=13.11, p<.01$, indicating a statistically significant difference between the Pre-assessment and Post-assessment of Conduct Problems. The mean for Pre-assessment (57.58) was significantly higher than the mean for Post-assessment (55.28) of Conduct Problems. Research Question 2

Do school-based mental health services with children and adolescents who receive Level 4 and Level 5 services who have Internalizing Problems change after interventions (individual counseling, group counseling, and/or family education)? Research Hypothesis 2 is that T Scores will decrease from Pre-assessment to Post-assessment for students who have Internalizing Problems and who receive individual counseling, group counseling, and/or family education services.

To test this hypothesis, a one-way, within subjects (repeated-measures) Analysis of Variance was computed in which the independent variable was Assessment Time (Preassessment versus Post-assessment) and the dependent variable was Internalizing Problems. This analysis yielded $F(1,345)=9.45, p<.01$, indicating a statistically significant difference between the Pre-assessment and Post-assessment of Internalizing Problems. The mean for Preassessment (55.77) was significantly higher than the mean for the Post-assessment (53.74) of Internalizing Problems.

An overall MANOVA was computed to include Research Question 2a, 2b, and 2c. In this MANOVA, the independent variable was Assessment Time (Pre-assessment versus Postassessment, and the dependent variables were Anxiety Problems, Depression Problems, and Somatization Problems. This analysis yielded a significant finding, $F(3,343)=3.53, p<.01$, indicating the need to conduct the three component Analyses of Variance. 
Research Question 2a. Do mental health services with children and adolescents who receive Level 4 and Level 5 services who have Anxiety Problems change after interventions (individual counseling, group counseling, and/or family education)? Research Hypothesis 2a is that T Scores will decrease from Pre assessment to Post assessment for students who have anxiety problems and who receive individual counseling, group counseling, and/or family education services.

This hypothesis was tested by computing a one-way, within-subjects (repeated-measures) Analysis of Variance in which the independent variable was Assessment Time (Pre-assessment versus Post-assessment), and the dependent variable was Anxiety Problems. This analysis yielded $F(1,345)=4.55, p<.05$, indicating a statistically significant difference between the Pre-assessment and Post-assessment of Anxiety Problems. The mean for Pre-assessment (53.14) was significantly higher than the mean for Post-assessment (51.62) of Anxiety Problems.

Research Question 2b. Do mental health services with children and adolescents who receive Level 4 and Level 5 services who have Depression Problems change after interventions (individual counseling, group counseling, and/or family education)? Research Hypothesis $2 \mathrm{~b}$ is that T Scores will decrease from Pre assessment to Post assessment for students who have depression problems and who receive individual counseling, group counseling, and/or family education services.

This hypothesis was tested by computing a one-way, within-subjects (repeated-measures) Analysis of Variance in which the independent variable was Assessment Time (Pre-assessment versus Post-assessment), and the dependent variable was Depression Problems. This analysis yielded $F(1,345)=9.94, p<.01$, indicating a statistically significant difference between the Pre-assessment and Post-assessment of Depression Problems. The mean for Pre-assessment 
(58.11) was significantly higher than the mean for Post-assessment (55.85) of Depression Problems.

Research Question 2c. Do mental health services with children and adolescents who receive Level 4 and Level 5 services who have Somatization Problems change after interventions (individual counseling, group counseling, and/or family education)? Research Hypothesis 1 is that T Scores will decrease from Pre assessment to Post assessment for students who have somatization problems and who receive individual counseling, group counseling, and/or family education services.

This hypothesis was tested by computing a one-way, within-subjects (repeated-measures) Analysis of Variance in which the independent variable was Assessment Time (Pre-assessment versus Post-assessment), and the dependent variable was Somatization Problems. This analysis yielded $F(1,345)=2.82, p<.10$, indicating a trend toward significance between the Preassessment and Post-assessment of Somatization Problems. The mean for Pre-assessment (52.47) was higher than the mean for Post-assessment (51.38) of Somatization Problems.

\section{Research Question 3}

Do mental health services with children and adolescents who receive Level 4 and Level 5 services who have School Problems change after interventions (individual counseling, group counseling, and/or family education)? Research Hypothesis 3 is that T Scores will decrease from Pre assessment to Post assessment for students who have school problems and who receive individual counseling, group counseling, and/or family education services.

To test this hypothesis, a one-way, within subjects (repeated-measures) Analysis of Variance was computed in which the independent variable was Assessment Time (Preassessment versus Post-assessment), and the dependent variable was School Problems. This 
analysis yielded $F(1,345)=6.69, p<.01$, indicating a statistically significant difference between the Pre-assessment and Post-assessment of School Problems. The mean for Preassessment (57.49) was significantly higher than the mean for the Post-assessment (56.22) of School Problems.

An overall MANOVA was computed to include Research Question 3a and 3b. In this MANOVA, the independent variable was Assessment Time (Pre-assessment versus Postassessment, and the dependent variables were Attention Problems and Learning Problems. This analysis yielded a significant finding, $F(2,344)=3.90, p<.05$, indicating the need to conduct the three component Analyses of Variance.

Research Question 3a. Do mental health services with children and adolescents who receive Level 4 and Level 5 services who have Attention Problems change after interventions (individual counseling, group counseling, and/or family education)? Research Hypothesis 3a is that T Scores will decrease from Pre assessment to Post assessment for students who have attention problems and who receive individual counseling, group counseling, and/or family education services.

This hypothesis was tested by computing a one-way, within-subjects (repeated-measures) Analysis of Variance in which the independent variable was Assessment Time (Pre-assessment versus Post-assessment), and the dependent variable was Attention Problems. This analysis yielded $F(1,345)=7.77, p<.01$, indicating a statistically significant difference between the Pre-assessment and Post-assessment of Attention Problems. The mean for Pre-assessment (58.19) was significantly higher than the mean for the Post-assessment (56.75) of Attention Problems. 
Research Question 3b. Do mental health services with children and adolescents who receive Level 4 and Level 5 services who have Learning Problems change after interventions (individual counseling, group counseling, and/or family education)? Research Hypothesis $3 \mathrm{~b}$ is that T Scores will decrease from Pre assessment to Post assessment for students who have learning problems and who receive individual counseling, group counseling, and/or family education services.

This hypothesis was tested by computing a one-way, within-subjects (repeated-measures) Analysis of Variance in which the independent variable was Assessment Time (Pre-assessment versus Post-assessment), and the dependent variable was Learning Problems. This analysis yielded $F(1,345)=2.76, p<.10$, indicating a trend toward significance between the Preassessment and Post-assessment of Learning Problems. The mean for Pre-assessment (55.47) was higher than the mean for the Post-assessment (54.61) of Learning Problems.

\section{Research Question 4}

Do mental health services with children and adolescents who receive Level 4 and Level 5 services who have Adaptive Skills Deficits change after interventions (individual counseling, group counseling, and/or family education)? Research Hypothesis 4 is that T Scores will increase from Pre assessment to Post assessment for students who have adaptive skills deficits and who receive individual counseling, group counseling, and/or family education services.

To test this hypothesis, a one-way, within subjects (repeated-measures) Analysis of Variance was computed in which the independent variable was Assessment Time (Preassessment versus Post-assessment), and the dependent variable was Adaptive Skills Deficits. This analysis yielded $F(1,345)=3.33, p<.10$, indicating a trend toward significance between the Pre-assessment and Post-assessment of Adaptive Skills Deficits. The mean for Pre- 
assessment (42.23) was higher than the mean for the Post-assessment (43.06) of Adaptive Skills Deficits.

An overall MANOVA was computed to include Research Question 4a, 4b, 4c, 4d, and 4e. In this MANOVA, the independent variable was Assessment Time (Pre-assessment versus Post-assessment, and the dependent variables were Adaptability Deficits, Social Skills Deficits, Leadership Deficits, Study Skills Deficits, and Functional Communication Deficits. This analysis yielded a trend toward significance in findings, $F(5,341)=2.09, p<.10$, indicating the need to conduct the three component Analyses of Variance.

Research Question 4a. Do mental health services with children and adolescents who receive Level 4 and Level 5 services who have Adaptability Deficits change after interventions (individual counseling, group counseling, and/or family education)? Research Hypothesis 4a is that T Scores will increase from Pre assessment to Post assessment for students who have adaptability deficits and who receive individual counseling, group counseling, and/or family education services.

This hypothesis was tested by computing a one-way, within-subjects (repeated-measures) Analysis of Variance in which the independent variable was Assessment Time (Pre-assessment versus Post-assessment), and the dependent variable was Adaptability Deficits. This analysis yielded $F(1,345)=8.07, p<.01$, indicating a statistically significant difference between the Pre-assessment and Post-assessment of Adaptability Deficits. The mean for Pre-assessment (41.83) was significantly lower than the mean for the Post-assessment (43.33) of Adaptability Deficits.

Research Question 4b. Do mental health services with children and adolescents who receive Level 4 and Level 5 services who have Social Skills Deficits change after interventions 
(individual counseling, group counseling, and/or family education)? Research Hypothesis $4 \mathrm{~b}$ is that T Scores will increase from Pre assessment to Post assessment for students who have social skills deficits and who receive individual counseling, group counseling, and/or family education services.

This hypothesis was tested by computing a one-way, within-subjects (repeated-measures) Analysis of Variance in which the independent variable was Assessment Time (Pre-assessment versus Post-assessment), and the dependent variable was Social Skills Deficits. This analysis yielded $F(1,345)=1.67, p>.10$, indicating a non-significant difference between the Preassessment and Post-assessment of Social Skills Deficits. The mean for Pre-assessment (43.15) was lower than the mean for the Post-assessment (43.79) of Social Skills Deficits.

Research Question 4c. Do mental health services with children and adolescents who receive Level 4 and Level 5 services who have Leadership Deficits change after interventions (individual counseling, group counseling, and/or family education)? Research Hypothesis 4c is that T Scores will increase from Pre assessment to Post assessment for students who have leadership deficits and who receive individual counseling, group counseling, and/or family education services.

This hypothesis was tested by computing a one-way, within-subjects (repeated-measures) Analysis of Variance in which the independent variable was Assessment Time (Pre-assessment versus Post-assessment), and the dependent variable was Leadership Deficits. This analysis yielded $F(1,345)=0.54, p>.10$, indicating a non-significant difference between the Preassessment and Post-assessment of Leadership Deficits. The mean for Pre-assessment (44.92) was lower than the mean for the Post-assessment (45.24) of Leadership Deficits. 
Research Question 4d. Do mental health services with children and adolescents who receive Level 4 and Level 5 services who have Study Skills Deficits change after interventions (individual counseling, group counseling, and/or family education)? Research Hypothesis 4d is that T Scores will increase from Pre assessment to Post assessment for students who have study skills deficits and who receive individual counseling, group counseling, and/or family education services.

This hypothesis was tested by computing a one-way, within-subjects (repeated-measures) Analysis of Variance in which the independent variable was Assessment Time (Pre-assessment versus Post-assessment), and the dependent variable was Study Skills Deficits. This analysis yielded $F(1,345)=3.49, p<.10$, indicating a trend toward significance between the Preassessment and Post-assessment of Study Skills Deficits. The mean for Pre-assessment (42.45) was lower than the mean for the Post-assessment (43.28) of Study Skills Deficits.

Research Question 4e. Do mental health services with children and adolescents who receive Level 4 and Level 5 services who have Functional Communication Deficits change after interventions (individual counseling, group counseling, and/or family education)? Research Hypothesis 4e is that T Scores will increase from Pre assessment to Post assessment for students who have functional communication deficits and who receive individual counseling, group counseling, and/or family education services.

This hypothesis was tested by computing a one-way, within-subjects (repeated-measures) Analysis of Variance in which the independent variable was Assessment Time (Pre-assessment versus Post-assessment), and the dependent variable was Functional Communication Deficits. This analysis yielded $F(1,345)=2.97, p<.10$, indicating a trend toward significance between the Pre-assessment and Post-assessment of Functional Communication Deficits. The mean for 
Pre-assessment (42.95) was lower than the mean for the Post-assessment (43.84) of Functional Communication Deficits.

To answer Research Questions 5, 6, 7, and 8 Multiple Linear Regression Analyses were computed with intention to follow up each relevant finding with appropriate Pearson Correlations. Regression analyses were performed for each Scale Score change in $\mathrm{T}$ as the Criterion Variable. Thus, for Research Questions 5, 6, 7, and 8 the Criterion Variables were Hyperactivity, Aggression, and Conduct Problems for Research Question 5; Anxiety, Depression, and Somatization for Research Question 6; Attention Problems and Learning Problems for Research Question 7; and Adaptability, Social Skills, Leadership, Study Skills, and Functional Communication for Research Question 8. As may be noted in Table 4 in Appendix $\mathrm{B}$, the Predictor Variables in each of these regression analyses will be minutes of counseling service (individual counseling, group counseling, and family education). These Regression Analyses resulted in no significant findings.

Due to the lack of sensitivity of the findings after Regression Analyses, the Criterion Variables, or T Scores, were categorized into three categories: $0=$ Non-Significant, $1=$ At-Risk, and 2 Clinically Significant. These categories were examined at pre and post intervention using a Chi-Square Analysis. These categories were derived from examination of the interpretation of T Scores on the Behavior Assessment System for Children, Second Edition (BASC-2). The BASC-2 suggests interpreting T Scores for the Clinical Scales as follows: Below $60=$ Non-Significant, $60-69=$ At-Risk, and 70+ $=$ Clinically Significant. The Clinical Scales for the BASC-2 are: Externalizing Problems, Hyperactivity Problems, Aggression Problems, Conduct Problems, Internalizing Problems, Anxiety Problems, Depression Problems, Somatization Problems, School Problems, Attention Problems, and Learning Problems. The 
BASC-2 suggests interpreting T Scores for the Adaptive Scales as follows: $40+=$ NonSignificant, $31-40$ = At-Risk, and Below $30=$ Clinically Significant. The Adaptive Scales for the BASC-2 are: Adaptive Skills Deficits, Adaptability Deficits, Social Skills Deficits, Leadership Deficits, Study Skills Deficits, and Functional Communication Deficits. Research Question 5

What type of school-based mental health service (individual counseling, group counseling, and/or family education) has the greatest impact on change in T-scores on children with Externalizing Problems who receive Level 4 and Level 5 services? Research Hypothesis 5 is that T Scores will be impacted greatest from Pre assessment to Post assessment for students who have Externalizing Problems and receive individual counseling and family education.

The Pre assessment T Score for Externalizing Problems were categorized into $0=$ NonSignificant, 1 =At-Risk, and 2 = Clinically Significant. The Post assessment $\mathrm{T}$ Score for Externalizing Problems were categorized into $0=$ Non-Significant, $1=$ At-Risk, and $2=$ Clinically Significant. A comparison of the category counts was computed in a 3 by 3 ChiSquare matrix. This analysis yielded Chi-Square $=32.14, \mathrm{df}=4, p<.01$, indicating a statistically significant relationship between the Pre assessment T Score and Post assessment T Score for Externalizing Problems. This Chi-Square matrix is shown in Table 15 in Appendix B. As may be noted for Externalizing Problems, 24 students moved from the Clinically Significant category to Non-Significant category after counseling, also 19 students moved from the Clinically Significant category to the At-Risk category. Table 15 in Appendix B shows the Observed frequencies compared to the Expected frequencies in the Chi-Square analysis, demonstrating the cells that are contributing most to the significant Chi-Square. 
Research Question 5a. What type of school-based mental health service (individual counseling, group counseling, and/or family education) has the greatest impact on change in Tscores on children with Hyperactivity Problems who receive Level 4 and Level 5 services? Research Hypothesis 5a is that T Scores will be impacted greatest from Pre assessment to Post assessment for students who have Hyperactivity Problems and receive individual counseling and family education.

The Pre assessment T Score for Hyperactivity Problems were categorized into $0=$ NonSignificant, 1 = At-Risk, and 2 = Clinically Significant. The Post assessment $\mathrm{T}$ Score for Hyperactivity Problems were categorized into $0=$ Non-Significant, $1=$ At-Risk, and $2=$ Clinically Significant. A comparison of the category counts was computed in a 3 by 3 ChiSquare matrix. This analysis yielded Chi-Square $=67.58, \mathrm{df}=4, p<.01$, indicating a statistically significant relationship between the Pre assessment $\mathrm{T}$ Score and Post assessment $\mathrm{T}$ Score for Hyperactivity Problems. This Chi-Square matrix is shown in Table 16 in Appendix B. As may be noted for Hyperactivity Problems, 22 students moved from the Clinically Significant category to Non-Significant category after counseling, also 25 students moved from the Clinically Significant category to the At-Risk category. Table 16 in Appendix B shows the Observed frequencies compared to the Expected frequencies in the Chi-Square analysis, demonstrating the cells that are contributing most to the significant Chi-Square.

Research Question 5b. What type of school-based mental health service (individual counseling, group counseling, and/or family education) has the greatest impact on change in $\mathrm{T}$ scores on children with Aggression Problems who receive Level 4 and Level 5 services? Research Hypothesis $5 \mathrm{~b}$ is that $\mathrm{T}$ Scores will be impacted greatest from Pre assessment to Post 
assessment for students who have Aggression Problems and receive individual counseling and family education.

The Pre assessment T Score for Aggression Problems were categorized into $0=$ NonSignificant, 1 = At-Risk, and 2 = Clinically Significant. The Post assessment T Score for Aggression Problems were categorized into $0=$ Non-Significant, $1=$ At-Risk, and $2=$ Clinically Significant. A comparison of the category counts was computed in a 3 by 3 Chi-Square matrix. This analysis yielded Chi-Square $=88.30, \mathrm{df}=4, p<.01$, indicating a statistically significant relationship between the Pre assessment T Score and Post assessment T Score for Aggression Problems. This Chi-Square matrix is shown in Table 17 in Appendix B. As may be noted for Aggression Problems, 17 students moved from the Clinically Significant category to the NonSignificant category after counseling, also 14 students moved from the Clinically Significant category to the At-Risk category. Table 17 in Appendix B shows the Observed frequencies compared to the Expected frequencies in the Chi-Square analysis, demonstrating the cells that are contributing most to the significant Chi-Square.

Research Question 5c. What type of school-based mental health service (individual counseling, group counseling, and/or family education) has the greatest impact on change in Tscores on children with Conduct Problems who receive Level 4 and Level 5 services? Research Hypothesis 5c is that T Scores will be impacted greatest from Pre assessment to Post assessment for students who have Conduct Problems and receive individual counseling and family education.

The Pre assessment T Score for Conduct Problems were categorized into $0=$ NonSignificant, 1 = At-Risk, and 2 = Clinically Significant. The Post assessment T Score for Conduct Problems were categorized into $0=$ Non-Significant, $1=$ At-Risk, and $2=$ Clinically 
Significant. A comparison of the category counts was computed in a 3 by 3 Chi-Square matrix. This analysis yielded Chi-Square $=58.07, \mathrm{df}=4, p<.01$, indicating a statistically significant relationship between the Pre assessment T Score and Post assessment T Score for Conduct Problems. This Chi-Square matrix is shown in Table 18 in Appendix B. As may be noted for Conduct Problems, 23 students moved from the Clinically Significant category to NonSignificant category after counseling, also 17 students moved from the Clinically Significant category to the At-Risk category. Table 18 in Appendix B shows the Observed frequencies compared to the Expected frequencies in the Chi-Square analysis, demonstrating the cells that are contributing most to the significant Chi-Square.

\section{Research Question 6}

What type of school-based mental health service (individual counseling, group counseling, and/or family education) has the greatest impact on change in T-scores on children with Internalizing Problems who receive Level 4 and Level 5 services? Research Hypothesis 6 is that T Scores will be impacted greatest from Pre assessment to Post assessment for students who have Internalizing Problems and receive individual counseling.

The Pre assessment T Score for Internalizing Problems were categorized into $0=$ NonSignificant, 1 = At-Risk, and 2 = Clinically Significant. The Post assessment $\mathrm{T}$ Score for Internalizing Problems were categorized into $0=$ Non-Significant, $1=$ At-Risk, and $2=$ Clinically Significant. A comparison of the category counts was computed in a 3 by 3 ChiSquare matrix. This analysis yielded Chi-Square $=37.43, \mathrm{df}=4, p<.01$, indicating a statistically significant relationship between the Pre assessment T Score and Post assessment T Score for Internalizing Problems. This Chi-Square matrix is shown in Table 19 in Appendix B. As may be noted for Internalizing Problems, 22 students moved from the Clinically Significant 
category to Non-Significant category after counseling, also 10 students moved from the Clinically Significant category to the At-Risk category. Table 19 in Appendix B shows the Observed frequencies compared to the Expected frequencies in the Chi-Square analysis, demonstrating the cells that are contributing most to the significant Chi-Square.

Research Question 6a. What type of school-based mental health service (individual counseling, group counseling, and/or family education) has the greatest impact on change in Tscores on children with Anxiety Problems who receive Level 4 and Level 5 services? Research Hypothesis 6a is that T Scores will be impacted greatest from Pre assessment to Post assessment for students who have Anxiety problems and receive individual counseling.

The Pre assessment T Score for Anxiety Problems were categorized into $0=$ NonSignificant, 1 = At-Risk, and 2 = Clinically Significant. The Post assessment T Score for Anxiety Problems were categorized into $0=$ Non-Significant, $1=$ At-Risk, and $2=$ Clinically Significant. A comparison of the category counts was computed in a 3 by 3 Chi-Square matrix. This analysis yielded Chi-Square $=19.55, \mathrm{df}=4, p<.01$, indicating a statistically significant relationship between the Pre assessment T Score and Post assessment T Score for Anxiety Problems. This Chi-Square matrix is shown in Table 20 in Appendix B. As may be noted for Anxiety Problems, 13 students moved from the Clinically Significant category to NonSignificant category after counseling, also 6 students moved from the Clinically Significant category to the At-Risk category. Table 20 in Appendix B shows the Observed frequencies compared to the Expected frequencies in the Chi-Square analysis, demonstrating the cells that are contributing most to the significant Chi-Square.

Research Question 6b. What type of school-based mental health service (individual counseling, group counseling, and/or family education) has the greatest impact on change in T- 
scores on children with Depression Problems who receive Level 4 and Level 5 services? Research Hypothesis $6 \mathrm{~b}$ is that T Scores will be impacted greatest from Pre assessment to Post assessment for students who have Depression Problems and receive individual counseling.

The Pre assessment T Score for Depression Problems were categorized into $0=$ NonSignificant, 1 = At-Risk, and 2 = Clinically Significant. The Post assessment $\mathrm{T}$ Score for Depression Problems were categorized into $0=$ Non-Significant, $1=$ At-Risk, and $2=$ Clinically Significant. A comparison of the category counts was computed in a 3 by 3 Chi-Square matrix. This analysis yielded Chi-Square $=30.63, \mathrm{df}=4, p<.01$, indicating a statistically significant relationship between the Pre assessment T Score and Post assessment T Score for Depression Problems. This Chi-Square matrix is shown in Table 21 in Appendix B. As may be noted for Depression Problems, 21 students moved from the Clinically Significant category to NonSignificant category after counseling, also 13 students moved from the Clinically Significant category to the At-Risk category. Table 21 in Appendix B shows the Observed frequencies compared to the Expected frequencies in the Chi-Square analysis, demonstrating the cells that are contributing most to the significant Chi-Square.

Research Question 6c. What type of school-based mental health service (individual counseling, group counseling, and/or family education) has the greatest impact on change in Tscores on children with Somatization Problems who receive Level 4 and Level 5 services? Research Hypothesis 6c is that T Scores will be impacted greatest from Pre assessment to Post assessment for students who have Somatization Problems and receive individual counseling.

The Pre assessment T Score for Somatization Problems were categorized into $0=$ NonSignificant, 1 = At-Risk, and 2 = Clinically Significant. The Post assessment $\mathrm{T}$ Score for Somatization Problems were categorized into $0=$ Non-Significant, $1=$ At-Risk, and $2=$ 
Clinically Significant. A comparison of the category counts was computed in a 3 by 3 ChiSquare matrix. This analysis yielded Chi-Square $=48.85, \mathrm{df}=4, p<.01$, indicating a statistically significant relationship between the Pre assessment T Score and Post assessment T Score for Somatization Problems. This Chi-Square matrix is shown in Table 22 in Appendix B. As may be noted for Somatization Problems, 11 students moved from the Clinically Significant category to Non-Significant category after counseling, also 11 students moved from the Clinically Significant category to the At-Risk category. Table 22 in Appendix B shows the Observed frequencies compared to the Expected frequencies in the Chi-Square analysis, demonstrating the cells that are contributing most to the significant Chi-Square.

\section{Research Question 7}

What type of school-based mental health service (individual counseling, group counseling, and/or family education) has the greatest impact on change in T-scores on children with School Problems who receive Level 4 and Level 5 services? Research Hypothesis 7 is that T Scores will be impacted greatest from Pre assessment to Post assessment for students who have School Problems and receive group counseling.

The Pre assessment T Score for School Problems were categorized into $0=$ NonSignificant, 1 = At-Risk, and 2 = Clinically Significant. The Post assessment T Score for School Problems were categorized into $0=$ Non-Significant, $1=$ At-Risk, and $2=$ Clinically Significant. A comparison of the category counts was computed in a 3 by 3 Chi-Square matrix. This analysis yielded Chi-Square $=80.60, \mathrm{df}=4, p<.01$, indicating a statistically significant relationship between the Pre assessment T Score and Post assessment T Score for School Problems. This Chi-Square matrix is shown in Table 23 in Appendix B. As may be noted for School Problems, 9 students moved from the Clinically Significant category to Non-Significant category after 
counseling, also 14 students moved from the Clinically Significant category to the At-Risk category. Table 23 in Appendix B shows the Observed frequencies compared to the Expected frequencies in the Chi-Square analysis, demonstrating the cells that are contributing most to the significant Chi-Square.

Research Question 7a. What type of school-based mental health service (individual counseling, group counseling, and/or family education) has the greatest impact on change in Tscores on children with Attention Problems who receive Level 4 and Level 5 services? Research Hypothesis $7 \mathrm{a}$ is that $\mathrm{T}$ Scores will be impacted greatest from Pre assessment to Post assessment for students who have Attention Problems and receive group counseling.

The Pre assessment T Score for Attention Problems were categorized into $0=$ NonSignificant, 1 = At-Risk, and 2 = Clinically Significant. The Post assessment T Score for Attention Problems were categorized into $0=$ Non-Significant, $1=$ At-Risk, and $2=$ Clinically Significant. A comparison of the category counts was computed in a 3 by 3 Chi-Square matrix. This analysis yielded Chi-Square $=26.06, \mathrm{df}=4, p<.01$, indicating a statistically significant relationship between the Pre assessment T Score and Post assessment T Score for Attention Problems. This Chi-Square matrix is shown in Table 24 in Appendix B. As may be noted for Attention Problems, 6 students moved from the Clinically Significant category to NonSignificant category after counseling, also 11 students moved from the Clinically Significant category to the At-Risk category. Table 24 in Appendix B shows the Observed frequencies compared to the Expected frequencies in the Chi-Square analysis, demonstrating the cells that are contributing most to the significant Chi-Square.

Research Question 7b. What type of school-based mental health service (individual counseling, group counseling, and/or family education) has the greatest impact on change in T- 
scores on children with Learning Problems who receive Level 4 and Level 5 services? Research Hypothesis 5 is that T Scores will be impacted greatest from Pre assessment to Post assessment for students who have Learning Problems and receive group counseling.

The Pre assessment T Score for Learning Problems were categorized into $0=$ NonSignificant, 1 = At-Risk, and 2 = Clinically Significant. The Post assessment T Score for Learning Problems were categorized into $0=$ Non-Significant, $1=$ At-Risk, and $2=$ Clinically Significant. A comparison of the category counts was computed in a 3 by 3 Chi-Square matrix. This analysis yielded Chi-Square $=82.15, \mathrm{df}=4, p<.01$, indicating a statistically significant relationship between the Pre assessment T Score and Post assessment T Score for Learning Problems. This Chi-Square matrix is shown in Table 25 in Appendix B. As may be noted for Learning Problems, 9 students moved from the Clinically Significant category to NonSignificant category after counseling, also 10 students moved from the Clinically Significant category to the At-Risk category. Table 25 in Appendix B shows the Observed frequencies compared to the Expected frequencies in the Chi-Square analysis, demonstrating the cells that are contributing most to the significant Chi-Square.

\section{Research Question 8}

What type of school-based mental health service (individual counseling, group counseling, and/or family education) has the greatest impact on change in T-scores on children with Adaptive Skills Deficits who receive Level 4 and Level 5 services? Research Hypothesis 8 is that T Scores will be impacted greatest from Pre assessment to Post assessment for students who have Adaptive Skills Deficits and receive individual counseling and group counseling.

The Pre assessment T Score for Adaptive Skills Deficits were categorized into $0=$ NonSignificant, $1=$ At-Risk, and 2 = Clinically Significant. The Post assessment T Score for 
Adaptive Skills Deficits were categorized into $0=$ Non-Significant, $1=$ At-Risk, and $2=$ Clinically Significant. A comparison of the category counts was computed in a 3 by 3 ChiSquare matrix. This analysis yielded Chi-Square $=32.85, \mathrm{df}=4, p<.01$, indicating a statistically significant relationship between the Pre assessment T Score and Post assessment T Score for Adaptive Skills Deficits. This Chi-Square matrix is shown in Table 26 in Appendix B. As may be noted for Adaptive Skills Deficits, 7 students moved from the Clinically Significant category to Non-Significant category after counseling, also 11 students moved from the Clinically Significant category to the At-Risk category. Table 26 in Appendix B shows the Observed frequencies compared to the Expected frequencies in the Chi-Square analysis, demonstrating the cells that are contributing most to the significant Chi-Square.

Research Question 8a. What type of school-based mental health service (individual counseling, group counseling, and/or family education) has the greatest impact on change in Tscores on children with Adaptability Deficits who receive Level 4 and Level 5 services? Research Hypothesis 8a is that T Scores will be impacted greatest from Pre assessment to Post assessment for students who have Adaptability Deficits and receive individual counseling and group counseling.

The Pre assessment T Score for Adaptability Deficits were categorized into $0=$ NonSignificant, $1=$ At-Risk, and $2=$ Clinically Significant. The Post assessment T Score for Adaptability Deficits were categorized into $0=$ Non-Significant, $1=$ At-Risk, and $2=$ Clinically Significant. A comparison of the category counts was computed in a 3 by 3 Chi-Square matrix. This analysis yielded Chi-Square $=37.81, \mathrm{df}=4, p<.01$, indicating a statistically significant relationship between the Pre assessment T Score and Post assessment T Score for Adaptability Deficits. This Chi-Square matrix is shown in Table 27 in Appendix B. As may be noted for 
Adaptability Deficits, 14 students moved from the Clinically Significant category to NonSignificant category after counseling, also 15 students moved from the Clinically Significant category to the At-Risk category. Table 27 in Appendix B shows the Observed frequencies compared to the Expected frequencies in the Chi-Square analysis, demonstrating the cells that are contributing most to the significant Chi-Square.

Research Question $8 b$. What type of school-based mental health service (individual counseling, group counseling, and/or family education) has the greatest impact on change in $\mathrm{T}$ scores on children with Social Skills Deficits who receive Level 4 and Level 5 services? Research Hypothesis $8 \mathrm{~b}$ is that T Scores will be impacted greatest from Pre assessment to Post assessment for students who have Social Skills Deficits and receive individual counseling and group counseling.

The Pre assessment T Score for Social Skills Deficits were categorized into $0=$ NonSignificant, 1 = At-Risk, and 2 = Clinically Significant. The Post assessment $\mathrm{T}$ Score for Social Skills Deficits were categorized into $0=$ Non-Significant, $1=$ At-Risk, and $2=$ Clinically Significant. A comparison of the category counts was computed in a 3 by 3 Chi-Square matrix. This analysis yielded Chi-Square $=25.67, \mathrm{df}=4, p<.01$, indicating a statistically significant relationship between the Pre assessment T Score and Post assessment T Score for Social Skills Deficits. This Chi-Square matrix is shown in Table 28 in Appendix B. As may be noted for Social Skills Deficits, 8 students moved from the Clinically Significant category to NonSignificant category after counseling, also 17 students moved from the Clinically Significant category to the At-Risk category. Table 28 in Appendix B shows the Observed frequencies compared to the Expected frequencies in the Chi-Square analysis, demonstrating the cells that are contributing most to the significant Chi-Square. 
Research Question 8c. What type of school-based mental health service (individual counseling, group counseling, and/or family education) has the greatest impact on change in Tscores on children with Leadership Deficits who receive Level 4 and Level 5 services? Research Hypothesis 8c is that T Scores will be impacted greatest from Pre assessment to Post assessment for students who have Leadership Deficits and receive individual counseling and group counseling.

The Pre assessment T Score for Leadership Deficits were categorized into $0=$ NonSignificant, 1 = At-Risk, and 2 = Clinically Significant. The Post assessment $\mathrm{T}$ Score for Leadership Deficits were categorized into $0=$ Non-Significant, $1=$ At-Risk, and $2=$ Clinically Significant. A comparison of the category counts was computed in a 3 by 3 Chi-Square matrix. This analysis yielded Chi-Square $=27.73, \mathrm{df}=4, p<.01$, indicating a statistically significant relationship between the Pre assessment T Score and Post assessment T Score for Leadership Deficits. This Chi-Square matrix is shown in Table 29 in Appendix B. As may be noted for Leadership Deficits, 1 student moved from the Clinically Significant category to Non-Significant category after counseling, also only 1 student moved from the Clinically Significant category to the At-Risk category. Table 29 in Appendix B shows the Observed frequencies compared to the Expected frequencies in the Chi-Square analysis, demonstrating the cells that are contributing most to the significant Chi-Square.

Research Question 8d. What type of school-based mental health service (individual counseling, group counseling, and/or family education) has the greatest impact on change in $\mathrm{T}$ scores on children with Study Skills Deficits who receive Level 4 and Level 5 services? Research Hypothesis $8 \mathrm{~d}$ is that T Scores will be impacted greatest from Pre assessment to Post 
assessment for students who have Study Skills Deficits and receive individual counseling and group counseling.

The Pre assessment T Score for Study Skills Deficits were categorized into $0=$ NonSignificant, 1 = At-Risk, and 2 = Clinically Significant. The Post assessment T Score for Study Skills Deficits were categorized into $0=$ Non-Significant, $1=$ At-Risk, and $2=$ Clinically Significant. A comparison of the category counts was computed in a 3 by 3 Chi-Square matrix. This analysis yielded Chi-Square $=49.24, \mathrm{df}=4, p<.01$, indicating a statistically significant relationship between the Pre assessment T Score and Post assessment T Score for Study Skills Deficits. This Chi-Square matrix is shown in Table 30 in Appendix B. As may be noted for Study Skills Deficits, 3 students moved from the Clinically Significant category to NonSignificant category after counseling, also 11 students moved from the Clinically Significant category to the At-Risk category. Table 30 in Appendix B shows the Observed frequencies compared to the Expected frequencies in the Chi-Square analysis, demonstrating the cells that are contributing most to the significant Chi-Square.

Research Question 8e. What type of school-based mental health service (individual counseling, group counseling, and/or family education) has the greatest impact on change in Tscores on children with Functional Communication Deficits who receive Level 4 and Level 5 services? Research Hypothesis 8e is that T Scores will be impacted greatest from Pre assessment to Post assessment for students who have Functional Communication Deficits and receive individual counseling and group counseling.

The Pre assessment T Score for Functional Communication Deficits were categorized into $0=$ Non-Significant, $1=$ At-Risk, and $2=$ Clinically Significant. The Post assessment $\mathrm{T}$ Score for Functional Communication Deficits were categorized into $0=$ Non-Significant, $1=$ At- 
Risk, and 2 = Clinically Significant. A comparison of the category counts was computed in a 3 by 3 Chi-Square matrix. This analysis yielded Chi-Square $=42.55, \mathrm{df}=4, p<.01$, indicating a statistically significant relationship between the Pre assessment T Score and Post assessment T Score for Functional Communication Deficits. This Chi-Square matrix is shown in Table 31 in Appendix B. As may be noted for Functional Communication Deficits, 9 students moved from the Clinically Significant category to Non-Significant category after counseling, also 12 students moved from the Clinically Significant category to the At-Risk category. Table 31 in Appendix B shows the Observed frequencies compared to the Expected frequencies in the Chi-Square analysis, demonstrating the cells that are contributing most to the significant Chi-Square. 


\section{CHAPTER IV}

\section{DISCUSSION}

The primary purpose of this study was to test the effectiveness of school-based mental health services in Hawaii's public school system. This study's focus was on the relationship between change in students' T-Scores on the Behavior Assessment System for Children, Second Edition (BASC-2), Teacher Rating Scale (TRS) and types of counseling services (individual counseling, group counseling, and/or family education) provided to students by school-based mental heath providers. The goal of this study was to determine what relationship exists between these variables in order to assist counseling providers and IEP teams in developing specific programs and providing targeted interventions for students in the school setting. The findings of the research study support several of the hypotheses, lend support to some existing research, and shed light on new directions for future research.

The findings of this study support the notion that counseling services do positively impact a students overall functioning. This is evident in the change in BASC-2 T Scores from time of referral to "counseling as a related service" (i.e. pretest) to one-year follow-up (i.e. posttest). The results of this study indicate statistically significant findings for the change in $\mathrm{T}$ Scores from baseline to follow-up at one year for many of the clinical scales and composite scales of the BASC-2. The analyses yielded statistically significant findings for thirteen of the analyses at the $p<.01$ level. The analyses yielded statistically significant findings at the $p<.05$ level for the overall MANOVA for Attention Problems and Learning Problems (Research Questions 3a and 3b). The analyses yielded a trend toward significance, at the $p<.10$ level, for several of the comparisons including, Adaptive Skills Deficits composite (Research Question 4), Somatization Problems (Research Question 1c), Learning Problems (Research Question 3b), 
Adaptability Deficits, Social Skills Deficits, Leadership Deficits, Study Skills Deficits, and Functional Communication Deficits (Research Questions 4a, 4b, 4c, 4d, and 4e), Study Skills Deficits (Research Question 4d), and Functional Communication Deficits (Research Question 4e). The analyses yielded non-significant results for Social Skills Deficits $(p=.20)$ and Leadership Deficits $(p=.46)$.

The change in T Scores from baseline to one year follow-up in Hawaii's public school system are consistent with past findings by Porter et al. (2000), who found that school-based mental health services are particularly effective when they work as a team and provide a continuum of care and integrate services in the school setting. Hawaii's public school system has utilized this principle to create a Comprehensive Student Support System (CSSS) to effectively serve all students in the state. Although this study's focus was on students receiving Level 4 and Level 5 services, it is likely that counseling services would exhibit similar results for students at lower levels of service (i.e. Level 3) due to the strong statistically significant results for many of the clinical scales.

Relationship between Student Needs and School-Based Mental Health Services

As has been previously reported by Adelman and Taylor (2000), schools are mandated to educate, not provide mental health services. At the same time many states are recognizing the value of providing mental health services within the context of the school environment. Ringeisen, Henderson, and Hoagwood (2003) found that schools play a critical role in the delivery of mental health services for students. School-based counseling services are now becoming more widely used to meet the student's needs (Slade, 2003).

Hawaii's Department of Education (HDOE) has developed a Comprehensive Student Support System (CSSS) to address the mental health needs of all of the students in the state of 
Hawaii. Carton and Weiss (1994) found that students with mental health needs identified in school are more likely to enter and receive treatment when mental health services are offered in school rather than when services are offered within the community. HDOE's CSSS follows this trend in the research allowing for early identification and intervention for all students.

The students who receive "counseling as a related service" as part of their IEP and are currently identified as being in need of Level 4 and Level 5 services through the CSSS. These are students who are first identified as being in need of "counseling as a related service" in the first quarter (January, February, and March) of 2005.

\section{Comparison of T Scores and Relationship to Intervention for Clinical Scales}

It was hypothesized that T Scores would decrease from Pre-assessment to Postassessment for students who have elevations on clinical subscales and clinical composite scales of the BASC-2 (Reynolds \& Kamphaus, 2002; Reynolds \& Kamphaus, 2004; Pumariega \& Vance, 1999; Porter, Epp, \& Bryan, 2000; Rones \& Hoagwood, 2000). The composite scales include: Externalizing Problems, Internalizing Problems, and School Problems. The clinical subscales include: Hyperactivity Problems, Aggression Problems, Conduct Problems, Anxiety Problems, Depression Problems, Somatization Problems, Attention Problems, and Learning Problems.

The analysis of the results of change in T Scores on the BASC-2 indicate the most significant statistical change in T Score for the clinical scales of the BASC-2. Analyses of clinical scales yielded statistically significant results in nearly all clinical scales. The results are consistent with pervious research (Porter et al., 2000) that school-based mental health services are effective. A plausible and potential interpretation of the results of this study is the support or the need for counseling services in the school-setting. This interpretation is the most plausible 
way to view these findings, as the students emotional and behavioral functioning were identified through the CSSS and interventions were targeted to address those specific concerns. It has been documented in previous research (Rones and Hoagwood, 2000) that although one-fifth of children in the United States have a diagnosable mental disorder, only about $16 \%$ of them ever receive any type of mental health service. Providing specific interventions within the context of the school is the most plausible conclusion to draw from these results. Often schools, according to Knitzer, Steinberg and Fleisch (1991), are reluctant to officially refer students for counseling. The authors found that schools are ultimately financially responsible for these services if they are included in a student's IEP. Instead, Knitzer et al., (1991) found that schools generally will request that parents seek out counseling services on their own, where the authors also found that little collaboration is present between the school and the mental health provider. The students in this study are officially referred for counseling services by the school and the counseling is part of the student's IEP.

Some may argue that maturation is another potential rationale for the change in $\mathrm{T}$ Scores. This is an unlikely rationale for the change seen in T Scores after intervention. Although the students in this study did mature over a one year period, it is unlikely that the number of clinical scales would change at such a statistically significant level if the change was due to maturational change. It is much more plausible that the change occurred due to the specific interventions for each student.

History is another factor that is a potential threat to internal validity for this study. The students in this study have been exposed to an unknown amount of additional factors over the year between data collection points. The extent of additional factors that the students have been exposed to are unknown and one could only speculate as to the extent of these historical factors. 
These may include, family changes (i.e. divorce), addition of medication, exposure to a different teacher, or outside peer activities.

Instrumentation is another potential and realistic threat to internal validity for the current study. Instrumentation refers to a change, not due to interventions, but rather to some change in the instrument used to measure the change. In this study the instrumentation concern is directly related to the fact that the two data points were collected by different teachers over a one year period. The BASC-2 attempts to control for internal validity, but this is a difficult issue to control, as it is based on teacher perceptions and observations of the student.

Regression to the mean is another potential threat to internal validity for this study. Regression to the mean refers to students who have extreme scores have a general tendency to regress to the mean over time, and intervention has little direct impact on this process. This concept has implications for this study as all of the students included in the study are receiving Level 4 and Level 5 services and have the potential to have extremely high scores on some of the Composite and Subscale scores. This threat to validity of regression to the mean is unlikely to be an accurate rationale for the changes found in this study, as the strong statistical significance suggests intervention is a more likely rationale.

The exception was the change in T Score for the clinical scales was for Somatization Problems. The Somatization Problems Scale, according to Reynolds and Kamphaus (2002), will typically show elevations for two reasons. First the scale will indicate the presence of a specific physical condition (i.e. diabetes, acute lymphocytic leukemia, muscular dystrophy, juvenile diabetes, and other conditions). Secondly, the Somatization Problems Scale will indicate the presence of physical complaints attributed to a mental health condition, including somatic complaints associated with depression and anxiety. There are two plausible and potential 
rationales for no strong statistically significant change in T Score for Somatization Problems. First, students with specific physical conditions, particularly of the variety found to show significance on the BASC-2, are not likely to show significant change over a one year period. The students in this study were referred for Level 4 and Level 5 services, primarily due to their behavioral and emotional conditions. The interventions are targeting these difficulties not physical conditions. A second rationale is that the change in somatic complaints attributed to a mental health condition, measured by the BASC-2, may not be sensitive enough to accurately detect this change, particularly with multiple raters involved. Often, somatic complaints attributed to a mental health condition, may be dismissed as physical complaints and many teachers may not make a connection between somatic complaints and mental health conditions. Comparison of T Scores and Relationship to Intervention for Adaptive Scales

It was hypothesized that T Scores would increase from Pre-assessment to Postassessment for students who have elevations on adaptive subscales and adaptive composite scale of the BASC-2 (Reynolds \& Kamphaus, 2002; Reynolds \& Kamphaus, 2004; Pumariega \& Vance, 1999; Porter, Epp, \& Bryan, 2000; Rones \& Hoagwood, 2000). The composite scale is the Adaptive Skills Scale. The adaptive subscales include: Adaptability, Social Skills, Leadership, Study Skills, and Functional Communication.

The BASC-2 includes six adaptive scales that measure positive behaviors of students (Reynolds \& Kamphaus, 2004). The analyses of the results of this study indicate less correlation between the change in $\mathrm{T}$ Score and the counseling interventions. A review of the results of the analyses of the change in T Score for the composite scale of Adaptive Skills, the overall MANOVA for change in T Score for Adaptability Deficits, Social Skills Deficits, Leadership Deficits, Study Skills Deficits, and Functional Communication Deficits, the change in T Score 
for Study Skills Deficits, and the change in T Score for Functional Communication Deficits indicate a trend toward significance $(p<.10)$. Analyses of the results also indicate that the change in T Score for Social Skills Deficits and the change in T Score for Leadership Deficits indicate no significant change. Analysis of results for Adaptability Deficits indicates statistically significant results. This was the only adaptive scale to demonstrate statistically significant findings.

Broadly examining these findings for the adaptive scales as compared to the clinical scales provides a plausible explanation for counseling interventions better addressing problem behaviors rather than building adaptive skills. This is a plausible rationale as counseling services are typically problem-focused and are typically initiated due to emotional and/or behavioral difficulties. Although building counseling interventions on the student's strengths has become the standard of care, interventions are still initiated based on problems experienced by the student. Counseling tends to focus on controlling overt behaviors; this alone does not necessarily build new adaptive skills in the students. Examining only the disruptive behaviors of students can be misleading for professionals, as long-term change is not likely with only extinguishing disruptive behaviors. Students need to demonstrate building adaptive skills to adequately replace disruptive behaviors, in order for long-term change to take place. The results demonstrated limited significance in improvement in Adaptive Skills. This is an important factor to consider as clinicians determine if counseling services are necessary to continue with identified students. One could hypothesize, although the Clinical Scales demonstrated statistically significant improvements, an anticipated behavioral relapse or regression in the future since there were limited improvement in Adaptive Skills. As counseling was initiated to address problem behaviors, it likely should continue to build Adaptive Skills. As counseling 
adequately addresses problem behaviors, counseling providers may be best serving their students by beginning to incorporate solution-based strategies to assist in building Adaptive Skills. Counseling providers could continue to monitor student progress, particularly around the building of Adaptive Skills.

Examining the change in T Scores for the Adaptive Skills scale indicates a trend toward significance. The Adaptive Skills scale is a composite scale made up of five adaptive scales which, according to Reynolds and Kampahus (2004) measures “appropriate emotional expression and control, daily-living skills inside and outside the home, and communication skills, as well as prosocial, organizational, study, and other adaptive skills" (p. 67). A plausible explanation is that these Adaptive Skills do not make significant improvements with the implementation of counseling services. Providers of counseling services may examine interventions, similar to Stress Inoculation Training (Meichenbaum, 1996), that integrates both cognitive and affective factors related to the coping process to build adaptive skills in individuals.

Investigating the analysis of the change in T Scores for the Study Skills scale reveals that this scale also demonstrates a trend toward significance. One plausible explanation for these results is that the students included in this study are students who are having significant emotional and behavioral problems. The items listed on the Study Skills scale relate directly to homework completion, reading assignments, and organizational skills (Reynolds \& Kamphaus, 2002). These skills are ones typically possessed by students who are functioning well academically. Academic success and emotional and behavioral well-being are typically closely related and students who are not functioning well emotionally or behaviorally likely do not possess strong study skills. The change in study skills is likely not detectable with students who 
are experiencing significant emotional and behavioral problems, such as the students in this study.

Examining the analysis of the change in T Scores for the Functional Communication scale reveals that this scale demonstrated a trend toward significance. The Functional Communication scale, according to Reynolds and Kamphaus (2004), “assesses the child's ability to express ideas and communicate in ways that others can easily understand" (p. 64). One potential explanation for these results is that students who are having significant emotional and behavioral problems may be experiencing problems with Functional Communication. These students may have difficulty adequately expressing themselves accurately and appropriately. As students begin to benefit from counseling they may begin to demonstrate more adaptive functional communication, hence the trend toward significance in the results of this study, as the students in this study are receiving an intensive level of services due to their functioning.

The Adaptability scale is the only adaptive scale that demonstrated statistically significant change. This may be due to the sensitivity of the scale, particularly for students with severe disruptive behaviors (Reynolds \& Kamphaus, 2002). This scale will generally indicate when a student has problems with flexibility and transitions. These behaviors are often addressed in the school setting by providing additional structure and preparing students for transitions with verbal and non-verbal cues (Reynolds \& Kamphaus, 2004). The students included in this study may have had additional interventions to address adaptability, which may have contributed directly to the significant change in T Scores. Additional variables were not available for further analyses related to these possibilities.

Examination of the analyses of two of the adaptive scales indicated no significant results. These were the Leadership and Social Skills scales. There are several plausible and potentially 
likely explanations for these results, including the limited sample of students likely in the sample with high Leadership skills. Reynolds and Kamphaus (2002) describe the Leadership scale as one that includes students who are high-achieving. The sample of students used for this study does not lend itself to a large enough sample of high-achieving students to detect a statistically significant change in T Scores. The Social Skills scale can be used to assist clinicians in differentiating between mental retardation and autism, both groups were not included in the sample for this study. The changes in T Scores would not have produced enough of an effect to detect a change that was statistically significant.

\section{Specific Counseling Services}

Students were identified as being in need of "counseling as a related service" as part of their IEP due to identified emotional and behavioral concerns. Counseling providers were assigned to students in need of counseling services. Each individual IEP team determined a specific number of counseling minutes for each student and included this on the student's IEP. Counseling providers are required to log actual counseling minutes provided to each student, as part of the SBBH Program Monitoring for each student (see Attachment A). Minutes are logged on a spread sheet and categorized into three categories: Individual Counseling, Group Counseling, and Family Education.

Individual Counseling is commonly known as a one-to-one intervention between a counseling provider and a student. Individual counseling minutes are recorded by the counseling provider at a time of contact with the student. Individual counseling may refer to traditional individual counseling sessions as well as times when a counseling provider engages in a less structured conversation with a student. Individual counseling is occurring when a counseling provider is meeting with a student directly to benefit that student by providing some direct 
intervention aimed at meeting goals of the student's IEP. Individual interventions can be to address a wide variety of issues for students, including, but not limited to depression, anxiety, school problems, learning problems, coping skills development, anger or aggressive problems, or social skills deficits. Individual counseling can also be used to assist in building adaptive or prosocial skills with students.

Group counseling is commonly known as group interventions with the student and other students who are targeted for direct service. Typically, group interventions are recognized when a counseling provider is providing direct intervention to a group (small or large) of students who are seeking intervention for addressing some needs in a group treatment modality. Group counseling interventions are occurring when a student in being provided with a direct counseling intervention to address a specific goal on the child's IEP. Group counseling interventions can be for a wide array of problems including, but not limited to, grief groups, study skills groups, divorce groups, and social skills groups.

Family education is the final category of service provided by the counseling providers at the Department of Education in Hawaii's public schools. This category is the proverbial catchall category for counseling providers. Family education can refer to the widest array of counseling services, and often implies that the services were consultative or collateral in nature and not providing a direct service to the student. Family education can imply that the counseling provider did meet with the family to address some issue of relevance to the student, but it may also imply that the counseling provider met with a teacher or someone else relevant to the student to address issues of relevance about the student. These services may include, but are not limited to, IEP meetings, student observations, teacher meetings, collection of student data, 
parent meetings, family meetings, or other indirect time a counseling provider spends working toward addressing specific goals on the students IEP.

Counseling providers vary widely between buildings and across islands in the state of Hawaii. Counseling providers include HDOE staff with a variety of job duties including clinical psychologists, school psychologists, school counselors, and school social workers. The educational level of counseling providers also varies widely between buildings and across islands in the state of Hawaii. Some counseling providers are doctoral level, others are masters prepared, and some were grandfathered into positions as counseling providers at the bachelor's level. Some of the counseling providers are licensed through state licensing boards as clinical psychologists, counselors, and social workers; others are licensed or certified through the Hawaii Department of Education as school psychologists, school counselors, and school social workers. There are other counseling providers that remain unlicensed. Training of counseling providers varies widely, with some trained within the state of Hawaii and others from around the country. Relationship of Specific Counseling Services to Change

It was hypothesized that T Scores would be impacted greatest from Pre assessment to Post assessment for students who have elevations on subscales and composite scales (including: Externalizing Problems, Hyperactivity Problems, Aggression Problems, and Conduct Problems) of the BASC-2 and receive individual counseling and family education (Reynolds \& Kamphaus, 2002; Reynolds \& Kamphaus, 2004; Chambless and Ollendick, 2001; Franklin, Harris, and Allen-Meares, 2006).

There was no support in these results for the hypotheses regarding the impact of individual counseling and family education on the decrease in T Scores for students who have Externalizing Problems, Hyperactivity Problems, Aggression Problems, and Conduct Problems. 
In other words, the specific counseling interventions (individual counseling, group counseling, and family education) did not appear to directly correlate to decrease in T Scores over a one year period. The investigators also examined the specific number of counseling minutes of each counseling intervention and also the total number of counseling minutes. None of these factors appeared to have a direct correlation to decreased T Scores for students who have Externalizing Problems, Hyperactivity Problems, Aggression Problems, and Conduct Problems.

It was hypothesized that T Scores would be impacted greatest from Pre assessment to Post assessment for students who have elevations on subscales and composite scales (including: Internalizing Problems, Anxiety Problems, Depression Problems, and Somatization Problems) of the BASC-2 and receive individual counseling (Reynolds \& Kamphaus, 2002; Reynolds \& Kamphaus, 2004; Chambless and Ollendick, 2001; Franklin, Harris, and Allen-Meares, 2006). There was no support in these results to support the hypotheses regarding the impact of individual counseling on the decrease in T Scores for students who have Internalizing Problems, Anxiety Problems, Depression Problems, and Somatization Problems. In other words, the specific counseling interventions (individual counseling, group counseling, and family education) did not appear to directly correlate to decrease in T Scores over a one year period. The investigators also examined the specific number of counseling minutes of each counseling intervention and also the total number of counseling minutes. None of these factors appeared to have a direct correlation to decreased T Scores for students who have Internalizing Problems, Anxiety Problems, Depression Problems, and Somatization Problems.

It was hypothesized that T Scores would be impacted greatest from Pre assessment to Post assessment for students who have elevations on subscales and composite scales (including: School Problems, Attention Problems, and Learning Problems) of the BASC-2 and receive group 
counseling (Reynolds \& Kamphaus, 2002; Reynolds \& Kamphaus, 2004; Chambless and Ollendick, 2001; Franklin, Harris, and Allen-Meares, 2006).

There was no support in these results for the hypotheses regarding the impact of individual counseling on the decrease in T Scores for students who have School Problems, Attention Problems, and Learning Problems. In other words, the specific counseling interventions (individual counseling, group counseling, and family education) did not appear to directly correlate to decrease in T Scores over a one year period. The investigators also examined the specific number of counseling minutes of each counseling intervention and also the total number of counseling minutes. None of these factors appeared to have a direct correlation to decreased T Scores for students who have School Problems, Attention Problems, and Learning Problems.

It was hypothesized that $\mathrm{T}$ Scores would be impacted greatest from Pre assessment to Post assessment for students who have elevations on subscales and composite scales (including: Adaptive Skills, Adaptability, Social Skills, Leadership, Study Skills, and Functional Communication) of the BASC-2 and receive individual counseling and group counseling (Reynolds \& Kamphaus, 2002; Reynolds \& Kamphaus, 2004).

There was no support in these results for the hypotheses regarding the impact of individual counseling on the increase in T Scores for students who have Adaptive Skills, Adaptability, Social Skills, Leadership, Study Skills, and Functional Communication was not supported. In other words, the specific counseling interventions (individual counseling, group counseling, and family education) did not appear to directly correlate to increase in $\mathrm{T}$ Scores over a one year period. The investigators also examined the specific number of counseling minutes of each counseling intervention and also the total number of counseling minutes. None 
of these factors appeared to have a direct correlation to decreased $\mathrm{T}$ Scores for students who have Adaptive Skills, Adaptability, Social Skills, Leadership, Study Skills, and Functional Communication.

Due to the lack of sensitivity of the findings after Regression Analyses, the Criterion Variables, or T Scores were categorized into three categories: Non-Significant, At-Risk, and Clinically Significant. These categories were derived by Reynolds and Kamphaus (2004) from examination of the interpretation of the BASC-2. Following Chi-Square analyses statistically significant findings were derived. This analysis of results shed further light on the significant change that is present after intervention, although the specific intervention does not appear to have a significant impact on the change. The specific interventions (individual counseling, group counseling, and family education) did not appear to have a direct impact or correlation to change in T Scores. One might hypothesize; however, that the nature of the counseling relationship itself directly correlated to the change in T Scores, and the specific intervention (individual counseling, group counseling, and family education) does not make a difference in the improvement.

Another potential explanation for the findings is the Pygmalion effect, also known as the Rosenthal effect, or the findings that leaders' expectations for subordinate performance can subsequently affect leader behavior and subordinate performance (White \& Locke, 2000). Since Rosenthal and Jacobson's publication in 1968 of teacher expectation effects (Feldman \& Prohaska, 1979), there is a strong body of literature to examine the phenomenon of teacher expectation effects (Feldman \& Prohaska, 1979; Feldman \& Theiss, 1982) and it application to other areas outside of the traditional classroom (Eden, 1984). 
The potential application of the Rosenthal effect to the findings of the current study, are the counselor and teacher have certain expectations about the students who are receiving counseling services. These expectations may include the expectation or belief that since these students were receiving Level 4 and Level 5 services they should progress more quickly than students receiving lower levels of service (i.e. Level 3). These expectations have been found in previous studies (Learman, Avorn, Everitt \& Rosenthal as cited in White \& Locke, 2000) to support measurable improvements in patient progress. The counseling providers have the potential to expect their interventions to provide positive outcomes for the students and this expectation may demonstrated the improvement found in this study. Teachers would also have the potential to expect change in students when they are aware that the students are receiving counseling services in the school setting. This potential explanation could also explain the reason for no correlation between the counseling minutes and the change in $\mathrm{T}$ Scores for the students in this study. This rationale was not an expected outcome, but after further analysis of the results, may indicate the reason for the lack of significant effect from the actual minutes of counseling.

Another potential explanation for the findings in this study are the core conditions of counseling described by Carl Rogers (Kirschenbaum \& Jourdan, 2005), including unconditional positive regard, empathic understanding, and congruence. These core conditions, although acknowledged as important by professionals in the field of counseling and psychotherapy, have been historically difficult to measure. Unconditional positive regard refers to the counselor accepting the client unconditionally and non-judgmentally (Kirschenbaum \& Jourdan, 2005). This condition of unconditional positive regard provides the client with an opportunity to freely explore thoughts and feelings without the danger of being judged, rejected, or condemned. 
Empathic understanding implies that the counselor accurately understands the client's thoughts, feelings, and more importantly the client's perspective. The counselor perceives the view of the client from the client's world. Congruence is the final core condition of counseling. Congruence refers to the counselor being authentic and genuine with the client. The client does not need to speculate about what the counselor is thinking or feeling; instead the counselor is authentic with the client during treatment. Together these core conditions allow the client to grow and strengthen their identity. These core conditions of counseling are a potential explanation for the statistically significant results found in this study. When taking into consideration the many factors with both the clients (unknown demographic factors, i.e. age, sex, grade) and the counseling providers (wide range of training and education) it appears that a potential rationale for the results may be the counseling relationship and the unknown factors associated with this special relationship.

\section{Conclusions and Implications}

Conclusions drawn from these results were made within the scope of the limitations and assumptions of the study. The present research indicates that counseling interventions (individual counseling, group counseling, and family education) do have a direct impact on change in T Scores for students in Hawaii's public school system that are receiving Level 4 and Level 5 services. The most noticeable change was related primarily to the clinical scales of the BASC-2, and appears to be less directly correlated to the adaptive scales. This conclusion is derived from the statistically significant findings in the change in $\mathrm{T}$ Scores for the clinical scales and less significant findings for the adaptive scales.

The counseling services in general (individual counseling, group counseling, and family education) appear to be directly correlated to this change in T Score, although the actual minutes 
of counseling a student received appears to be less correlated to the change. This conclusion is drawn from the lack of statistical significance found when directly examining the impact of the counseling minutes on the change in T Score.

Adaptive behaviors appear to show less change related to intervention. Adaptive skills do not necessarily improve with counseling interventions. Counseling interventions, both in this study and in general, are typically implemented to address problematic behaviors, not necessarily to build adaptive skills. Counseling interventions that are not solution-focused do not necessarily have an impact on building adaptive skills, instead problematic behaviors are suppressed and potential regression may ensue.

Several of the hypotheses proposed in this study were supported, several others were not supported. The hypotheses that were supported by the findings of this study (Research Questions 1, 2, 3, and 4) provide additional support for the growing body of literature that supports the implementation of school-based mental health services to provide better mental health care to school age children and early intervention for childhood disorders. The hypotheses that were not supported by the findings of this study (Research Questions 5, 6, 7, and 8) shed new light on the number of counseling minutes students receive and the less than significant findings indicated in this study. These findings were unexpected but provide some insight into the impact or lack impact that the number of counseling minutes have on improvement in students.

\section{Recommendations for Future Studies}

The following recommendations for further research are provided, based on the findings of this study: 
1. Future researchers should attempt to gather data on other levels of services students receive in the school setting (i.e. Level 1, Level 2, and Level 3) and compare these findings to the findings for the students who receive higher levels of service.

2. Future researchers should attempt to use a more generalizable sample population so that the results are more easily generalized across the population. When results are easily applied to multiple populations the significance of the results has greater implications for making treatment recommendations.

3. Future researchers should include additional demographic variables including age, grade, sex, educational setting, etc. to include in the data analysis. These variables may assist future researchers in drawing further conclusions from results.

\section{Limitations of Findings}

Conclusions suggested from these findings were made within the scope of the assumptions and limitations of the study. In interpreting the results, the following limitations should be considered:

1. This study was done with a very specialized population of students in Hawaii. This population presents particular limitations in regard to cultural issues of both students and professional staff. This findings, although extremely interesting, will be very difficult to generalize to the general public school setting. The generalizability is further complicated by the current education and mental health systems in Hawaii. This education and mental health system may be unique to Hawaii and due to financial reasons may be difficult to duplicate elsewhere.

2. This study was limited by the number of additional variables available to analyze, due to the data being archival and not within the control of the researcher. Additional variables, 
including age, sex, grade, educational setting, etc. would have provided additional analyses that may have shed additional light on the results.

3. This study was limited by the counseling providers recoding the number of counseling minutes in the counseling logs. The data used for this study was archival data and originally not collected for use in this study. Counseling providers originally collected the data used in this study for monitoring purposes after a class action law suit. The motivation for the counseling providers to accurately record the counseling minutes in the counseling log may have had been to satisfy the monitoring team and not necessarily to examine the effects of counseling on the change in T Scores. A cursory overview of the counseling log revealed a wide variance in number of counseling minutes and many students only receiving individual counseling services. This study was limited by the counseling providers accurately recording the counseling minutes. 


\section{REFERENCES}

Adelman, H. S. \& Taylor, L. (2000). Shaping the future of mental health in the schools. Psychology in the Schools, 37, $49-60$.

American Psychiatric Association. (2000). Diagnostic and statistical manual of mental disorders ( $4^{\text {th }}$ ed.) text revision. Washington DC: Author.

Bell, C. (2003). Addendum: A brief commentary on the making of the supplement to the Surgeon General's report on mental health. Culture, Medicine and Psychiatry, 27 (4), 499-503.

Bierman, K. L. (2003). Commentary: New models for school-based mental health services. School Psychology Review, 32(4), 525 - 529.

Casat, C. D., Sobolewski, J., Gordon, J., \& Rigsby, J. (1999). School-based mental health services (SBS): A pragmatic view of a program. Psychology in the Schools, 36(5), $403-$ 413.

Center for Mental Health in the Schools. (2000). Pioneer initiatives to reform education support programs. June 2000, $16-18$.

Chambless, D. L. \& Ollendick, T. H. (2001). Empirically supported psychological interventions: Controversies and evidence. Annual Review of Psychology, 52, 685 - 716.

Chow, J. (2002). Asian American and Pacific Islander mental health and substance abuse agencies: Organizational characteristics and service gaps. Administration and Policy in Mental Health, 30, $79-86$.

Compton, S. N., Burns, B. J., Egger, H. L., \& Robertson E. (2002). Review of the evidence base for treatment of childhood psychopathology: Internalizing disorders. Journal of Consulting and Clinical Psychology, 70 (6), 1240 -1266. 
Del Mundo, A. S., Pumariega, A. J., \& Vance, H. R. (1999). Psychopharmacology in schoolbased mental heath services. Psychology in the Schools, 36(5), $437-450$.

Doyle, A., Ostrander, R., Skare, S., Crosby, R. D., \& August, G. J. (1997). Convergent and criterion-related validity of the Behavior Assessment System for Children - Parent Rating Scale. Journal of Clinical Child Psychology, 26, 276 - 284.

Farmer, E. M. Z., Compton, S. N., Burns, B. J., \& Robertson E. (2002). Review of the evidence base for treatment of childhood psychopathology: Externalizing disorders. Journal of Consulting and Clinical Psychology, 70(6), 1267 - 1302.

Franklin, C., Harris, M., \& Allen-Meares, P. (2006). The School Services Sourcebook: A Guide for School-Based Professionals. New York: Oxford Press

Friedberg, R. D. \& McClure, J. M. (2002). Clinical practice of cognitive therapy with children and adolescents - the nuts and bolts. New York: Guilford Press.

Eden, D. (1984). Self-fulfilling prophecy as a management tool: Harnessing Pygmalion. Academy of Management Review, 9(1), 64 - 73.

Feldman, R. S. \& Prohaska, T. (1979). The student as Pygmalion: Effect of student expectation on the teacher. Journal of Educational Psychology, 71(4), 485 - 493.

Feldman, R. S. \& Theiss, A. J. (1982). The teacher and student as Pygmalions: Joint effects of teacher and student expectations. Journal of Educational Psychology, 74(2), 217 - 223.

Harrison, P. L. \& Oakland, T. (2003). Adaptive Behavior Assessment System, Second Edition (ABAS II). San Antonio, TX: Harcourt Assessment Inc.

Hart, E. L. \& Lahey, B. B. (1999). General child behavior rating scales. In D. Shaffer, C. P. Lucas, \& J. E. Richters (Eds.), Diagnostic Assessment in Child and Adolescent Psychopathology. New York: The Guilford Press. 
Hawaii State Department of Health. (2003). Healthy people healthy communities healthy islands. 2003 Annual Report, 8-12.

Heneghan, A. M. (1997). Availability of school health services for young children. Journal of School Health, 67, $327-332$.

Hoagwood, K. \& Erwin, H. D. (1997). Effectiveness of school-based mental health service for children: A 10-year research review. Journal of Child and Family Studies, 6(4), 435 451.

Hu, T., Snowden, L. R., Jerrell, J. M., \& Nguyen, T. D. (1991). Ethnic populations in public mental health: Services choice and level of use. American Journal of Public Health $81(11), 1429-1434$.

Kamphaus, R.W., Huberty, C. J., DiStefano, C., \& Petoskey, M. D. (1997). The typology of teacher-rated child behavior of a national U.S. sample. Journal of Abnormal Child Psychology, 25, $453-463$.

Keppel, J. (1991). Design and analysis: A researcher's handbook ( ${ }^{\text {rd }}$ Edition). Upper Saddle River, NJ: Prentice Hall.

Kirschenbaum, H. \& Jourdan, A. (2005). The current status of Carl Rogers and person-centered approach. Psychotherapy: Theory, Research, Practice, Training, 42(1), 37 -51.

Knitzer, J, Steinberg, Z, \& Fleisch B. (1991). Schools, children's mental health, and the advocacy challenge. Journal of Clinical Child Psychology, 20, 102 - 111.

Lau, A. S., Garland, A. F., Yeh, M., McCabe, K. M., Wood, P. A., \& Hough, R. L. (2004). Race/ethnicity and inter-informant agreement in assessing adolescent psychopathology. Journal of Emotional and Behavioral Disorders, 12(3), 145-156.

Lau, A. \& Zane, N. (2000). Examining the effects of ethnic-specific services: An analysis of 
cost-utilization and treatment outcome for Asian American clients. Journal of Community Psychology, 28(1), $63-77$.

Leong, F. T. L. (1994). Asian Americans' differential patterns of utilization of inpatient and outpatient public mental health services in Hawaii. Journal of Community Psychology, $22,82-96$.

Matsuoka, J. K., Breauz, C., \& Ryujin, D. H. (1997). National utilization of mental health services by Asian Americans / Pacific Islanders. Journal of Community Psychology, 25, $141-145$.

Meichenbaum, D. (1996). Stress inoculation training for coping with stressors. The Clinical Psychologist, 49, 4-7.

Merenda, P. F. (1996). BASC: Behavior Assessment System for Children. Measurement and Evaluation in Counseling and Development, 28, 229 - 232.

Nabors, L. A., Weist, M. D., Tashman, N. A., \& Myers, C. P. (1999). Quality assurance and school-based mental health services. Psychology in the Schools, 36(6), $485-493$.

Okazaki, S. (2000). Treatment delay among Asian-American patients with severe mental illness. American Journal of Orthopsychiatry, 70 (1), 58-64.

Palafox, N. A., Buenconsejo-Lum, L., Riklon, S., \& Waitzfelder, B. (2002). Improving health outcomes in diverse populations: Competency in cross-cultural research with indigenous Pacific Islander populations. Ethnicity \& Health, 7, 279 - 285.

Pfeiffer, S. I. \& Reddy, L. A. (1998). School-based mental health programs in the United States: Present status and a blueprint for the future. School Psychology Review, 27(1), 84 -97 .

Porter, G., Epp, L., \& Bryan, S. (2000). Collaboration among school mental health 
professionals: A necessity, not a luxury. Professional School Counseling, 3(5), 1096 2409.

Pumariega, J. H., \& Vance, H. R. (1999). School-based mental health services: The foundation of systems of care for children's mental health. Psychology in the School, 36(5), 371 378.

Reynolds, C. R. \& Kamphaus, R. W. (2004). Behavior Assessment System for Children, Second Edition (BASC-2). Circle Pines, MN: American Guidance Service.

Reynolds, C. R. \& Kamphaus, R. W. (2002). The Clinician's Guide to the Behavior Assessment System for Children (BASC). New York: Guildford Press.

Ringeisen, H., Henderson, K., \& Hoagwood, K. (2003). Context matters: Schools and the "research to practice gap" in children's mental health. School psychology Review, 32(2), $153-168$.

Robinson, K. \& Rapport, L. J. (2002). Outcomes of a school-based mental health program for youth with serious emotional disorders. Psychology in the Schools, 39(6), $661-675$.

Rones, M. \& Hoagwood, K. (2000). School-based mental health services: A research review. Clinical Child and Family Psychology Review, 3(4), 223 - 241.

Sanders, D. S. (1991). Future directions in social services: Asian and Pacific Islander perspectives. In N. Mokuau (Ed.), Handbook of Social Services for Asian and Pacific Islanders. Westport, CT: Greenwood Press.

Slade, E. P. (2003). The Relationship between school characteristics and the availability of mental health and related health serviced in middle and high schools in the United States. The Journal of Behavioral Health Services \& Research, 30(4), 382 - 392.

Snowden, L. R. \& Cheung, F. K. (1990). Use of inpatient mental health services by members of 
ethnic minority groups. American Psychologist, 45(3), 347-355.

Sue, S., Fujino, D. C., Hu, L., Takeuchi, D. T., \& Zane, N. W. S. (1991). Community mental health services for ethnic minority groups: A test of the cultural responsiveness hypothesis. Journal of Consulting and Clinical Psychology, 59(4), 533 - 540.

U.S. Bureau of the Census. (2000). Census 2000 data for the state of Hawaii. Retrieved December 1, 2004 from http://www.census.gov/census2000/states/hi.html.

Weist, M. D., Myers, C. P., Danforth, J., McNeil, D. W., Ollendick, T. H., \& Hawkins, R. (2000). Expanded school mental health services: Assessing needs related to school level and geography. Community Mental Health Journal, 36(3), 259 - 273.

White, S. S., \& Locke, E. A. (2000). Problems with the Pygmalion effect and some proposed solutions. Leadership Quarterly, 11(3), $389-415$.

Yeh, M., McCabe, K., Hough, R. L., Dupuis, D., \& Hazen A. (2003). Racial/ethnic differences in parental endorsement of barriers to mental health services for youth. Mental Health Services Research, 5(2), $65-77$. 


\section{Appendix A}

November 30, 2004

\section{ACTION REQUIRED}

To: $\quad$ Complex Area Superintendents, School Principals and District Educational Specialists

From: Patricia Hamamoto, Superintendent

Subject: School-Based Behavioral Health (SBBH) Program EvaluationImplementation of the Behavioral Assessment System for ChildrenSecond Edition (BASC-2)

Effective January 3, 2005, the Behavioral Assessment System for Children-Second Edition (BASC-2) will be utilized as a measurement for student progress as well as School-Based Behavioral Health (SBBH) program effectiveness. As noted in the attached implementation table, various components of the BASC-2 will be administered for students with emotional/behavioral concerns:

- When considering or initiating counseling as an Individualized Education Program (IEP) or Modification Plan (MP) related service;

- At the time of the annual review/re-evaluation for students who are receiving counseling as a related service; and thereafter,

- At the time of the Quarterly Progress Report, through 15 minutes structured observations of the student in the area of his or her difficulty; and

- When considering discontinuation of counseling as a related service.

The BASC-2, a multi-purpose tool specifically designed for use in the school setting, is one source of data that must be considered with additional information from other sources. With systematic administration, it is envisioned that, within a year, the BASC-2 will have been administered to every student who receives counseling as a related service. This will provide pre-, mid-, and end-of-services data for students and program monitoring. Consequently, the administration of the Achenbach System of Empirically Based Assessment (ASEBA) on a random sample of Felix-class students and the Internal Review sample will be discontinued immediately. 
Complex Area Superintendents, School Principals and District Educational Specialists November 22, 2004

Page 2

The attached BASC-2 implementation table identifies the specific components to be administered, the professionals who may be responsible for administering them, who is typically interviewed and who completes the forms. A more detailed procedure, including issues related to informed consent prior to administration of these scales, documentation of the use of rating scales for progress monitoring, and computer scoring of the scales will be provided to the SBBH District Educational Specialists by December 3, 2004.

The systematic use of the BASC-2 is expected to:

- Provide a clear focus on student emotional and behavioral needs;

- Provide more relevant information for making educational program decisions;

- Help identify the most effective interventions for the student;

- Provide an objective measure of student progress towards amelioration of his or her behavioral and emotional concerns; and

- Provide a measurement of SBBH program effectiveness.

The commitment to effectively serve students with emotional and behavioral needs through implementation of the BASC-2 by all Student Support Teams, IEP teams, MP teams and counseling providers is appreciated. Should you have any questions, please contact Ms. Paulie Schick, SBBH Educational Specialist, at 735-6225.

PH:PS:jm

Attachment

c: Assistant Superintendents

Branch Directors, Superintendent's Office

Public Charter School Offices Program Office

Office of Curriculum, Instruction and Student Support 


\section{BASC-2 Implementation}

\begin{tabular}{|c|c|c|c|}
\hline $\begin{array}{l}\text { Events Requiring } \\
\text { Administration of } \\
\text { BASC-2* }\end{array}$ & $\begin{array}{l}\text { Required Components of } \\
\text { BASC-2 and Source of } \\
\text { Information }\end{array}$ & $\begin{array}{l}\text { Other BASC-2 } \\
\text { Components } \\
\text { that may also be } \\
\text { Applicable }\end{array}$ & $\begin{array}{l}\text { Person responsible for } \\
\text { Administering BASC-2 } \\
\text { Components }\end{array}$ \\
\hline $\begin{array}{l}\text { 1) When considering or } \\
\text { initiating counseling as } \\
\text { an Individualized } \\
\text { Education Program } \\
\text { (IEP) or Modification } \\
\text { Plan (MP) related } \\
\text { service }\end{array}$ & $\begin{array}{l}\Rightarrow \text { Teacher Rating Scale } \\
\text { (TRS) } \\
\text { (Teacher, Educational Assistant, } \\
\text { other knowledgeable school staff) } \\
\Rightarrow \text { Student Observation } \\
\text { System (SOS) } \\
\text { (Classroom or other environment } \\
\text { of interest to identify needs or } \\
\text { strengths) } \\
\Rightarrow \text { Self-Report of } \\
\text { Personality (SRP) } \\
\text { (If student is 6 years-old or older) } \\
\text { The following are to be } \\
\text { completed within } 60 \text { days of } \\
\text { start of service: } \\
\Rightarrow \text { Parent Rating Scale } \\
\text { (PRS), if possible } \\
\text { (Parent, foster parent other } \\
\text { knowledgeable caregiver) } \\
\Rightarrow \text { Social-Developmental } \\
\text { History (SDH) }\end{array}$ & $\begin{array}{l}\text { N/A-The entire } \\
\text { system is required }\end{array}$ & $\begin{array}{l}\text { School Psychologist, Clinical } \\
\text { Psychologist, Behavioral } \\
\text { Specialist, Social Worker, } \\
\text { School Counselor or } \\
\text { Contracted Provider** } \\
\text { assigned to provide services }\end{array}$ \\
\hline $\begin{array}{l}\text { 2) Annual Review/Re- } \\
\text { evaluation of IEP/MP } \\
\text { for students who are } \\
\text { receiving counseling as } \\
\text { a related service }\end{array}$ & $\begin{aligned} \Rightarrow & \text { Social-Developmental } \\
& \text { History (SDH) - update } \\
\Rightarrow & \text { Teacher Rating Scale } \\
& \text { (TRS) } \\
\Rightarrow & \text { Student Observation } \\
& \text { System (SOS) }\end{aligned}$ & $\begin{aligned} & \Rightarrow \text { In current } \\
& \text { areas of } \\
& \text { concern or } \\
& \Rightarrow \text { Other BASC- } \\
& 2 \text { components } \\
& \text { that were } \\
& \text { significant } \\
& \text { during } \\
& \text { previous } \\
& \text { evaluations }\end{aligned}$ & $\begin{array}{l}\text { Behavioral Specialist, Social } \\
\text { Worker, School Counselor or } \\
\text { Contracted Provider** } \\
\text { assigned to provide services }\end{array}$ \\
\hline $\begin{array}{l}\text { 3) Quarterly Progress } \\
\text { Reports following 1or } 2 \\
\text { above }\end{array}$ & $\begin{array}{l}\Rightarrow \text { Student Observation } \\
\text { System (SOS) } \\
\text { in the area of student's } \\
\text { difficulty }\end{array}$ & 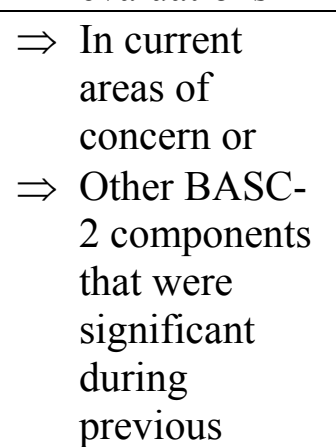 & $\begin{array}{l}\text { Behavioral Specialist, Social } \\
\text { Worker, School Counselor or } \\
\text { Contracted Provider** } \\
\text { assigned to provide services }\end{array}$ \\
\hline
\end{tabular}




\begin{tabular}{|l|l|l|l|}
\hline & & evaluations & \\
\hline $\begin{array}{l}\text { 4) When considering } \\
\text { discontinuation of } \\
\text { counseling as a related } \\
\text { service. }\end{array}$ & $\Rightarrow \begin{array}{l}\text { Teacher Rating Scale } \\
\text { (TRS) }\end{array}$ & $\begin{array}{l}\text { Other BASC- } \\
\text { Student Observation } \\
\text { System (SOS) } \\
\text { nemponents }\end{array}$ & $\begin{array}{l}\text { Behavioral Specialist, Social } \\
\text { successful } \\
\text { transition }\end{array}$ \\
\end{tabular}

* Specified BASC-2 component does not need re-administration if completed within 60 days prior to these events ** Contracted Providers will be completing the Student Observation System for assigned students per the Request for Proposals (RFP), effective July 1, 2005

Consultation for interpretation of findings and recommendations for intervention is provided by the clinical or school psychologist. 


\section{Appendix B}

Table 1

F - Index Raw Score Frequency Summary

\begin{tabular}{llll}
\hline & & & \\
& Acceptable & Caution & Extreme Caution \\
TRS-P & $94.1 \%$ & $4.4 \%$ & $1.5 \%$ \\
& $(0-1)$ & $(2-3)$ & $(4-20)$ \\
TRS-C & $96.9 \%$ & $1.9 \%$ & $1.2 \%$ \\
& $(0-1)$ & $(2-3)$ & $(4-20)$ \\
TRS-A & $97.1 \%$ & $1.9 \%$ & $1.0 \%$ \\
& $(0-1)$ & $(2-3)$ & $(4-20)$ \\
\hline
\end{tabular}

Note: Raw score ranges are provide in parentheses

Adapted from Reynolds and Kamphaus (2004) (p. 110, Table 10.5) 
Table 2

Clinical and Adaptive Composite and Scale Scores

\begin{tabular}{lcc}
\hline & Clinical Scales & \\
& Adaptive Scales \\
Composite & Externalizing Problems & Adaptive Skills \\
Scale & Hyperactivity & Adaptability \\
& Aggression & Social Skills \\
& Conduct Problems & Leadership \\
& & Study Skills \\
Composite & & Functional Communication \\
Scale & Internalizing & \\
& Anxiety & \\
Depression & \\
Composite & Somatization & \\
Scale & School Problems & \\
& Attention Problems & \\
& Learning Problems & \\
\hline
\end{tabular}

Table adapted from Reynolds and Kamphaus (2004) 
Table 3

Independent and Dependent Variables for Research Questions 1, 2, 3, and 4

\begin{tabular}{|c|c|c|c|}
\hline Research Question & Independent Variable & $\begin{array}{c}\text { Dependent Variable } \\
(\mathrm{T}-\mathrm{Score})\end{array}$ & Analysis Strategy \\
\hline & & Composite Scores & \\
\hline RQ1 & $\begin{array}{l}\text { Assessment Time } \\
\text { (Pre vs. Post) }\end{array}$ & Externalizing Problems & $\begin{array}{l}\text { MANOVA } 1 \\
\text { ANOVA }\end{array}$ \\
\hline RQ2 & $\begin{array}{l}\text { Assessment Time } \\
\text { (Pre vs. Post) }\end{array}$ & Internalizing Problems & $\begin{array}{l}\text { MANOVA } 1 \\
\text { ANOVA }\end{array}$ \\
\hline RQ3 & $\begin{array}{l}\text { Assessment Time } \\
\text { (Pre vs. Post) }\end{array}$ & School Problems & $\begin{array}{l}\text { MANOVA } 1 \\
\text { ANOVA }\end{array}$ \\
\hline \multirow[t]{2}{*}{ RQ4 } & $\begin{array}{l}\text { Assessment Time } \\
\text { (Pre vs. Post) }\end{array}$ & Adaptive Skills & $\begin{array}{l}\text { MANOVA } 1 \\
\text { ANOVA }\end{array}$ \\
\hline & & Scale Scores & \\
\hline RQ1a & $\begin{array}{l}\text { Assessment Time } \\
\text { (Pre vs. Post) }\end{array}$ & Hyperactivity & $\begin{array}{l}\text { MANOVA } 2 \\
\text { ANOVA }\end{array}$ \\
\hline RQ1b & $\begin{array}{l}\text { Assessment Time } \\
\text { (Pre vs. Post) }\end{array}$ & Aggression & $\begin{array}{l}\text { MANOVA } 2 \\
\text { ANOVA }\end{array}$ \\
\hline \multirow[t]{2}{*}{ RQ1c } & $\begin{array}{l}\text { Assessment Time } \\
\text { (Pre vs. Post) }\end{array}$ & Conduct Problems & $\begin{array}{l}\text { MANOVA } 2 \\
\text { ANOVA }\end{array}$ \\
\hline & & Scale Scores & \\
\hline RQ2a & $\begin{array}{l}\text { Assessment Time } \\
\text { (Pre vs. Post) }\end{array}$ & Anxiety & $\begin{array}{l}\text { MANOVA } 3 \\
\text { ANOVA }\end{array}$ \\
\hline RQ2b & $\begin{array}{l}\text { Assessment Time } \\
\text { (Pre vs. Post) }\end{array}$ & Depression & $\begin{array}{l}\text { MANOVA } 3 \\
\text { ANOVA }\end{array}$ \\
\hline RQ2c & $\begin{array}{l}\text { Assessment Time } \\
\text { (Pre vs. Post) }\end{array}$ & Somatization & $\begin{array}{l}\text { MANOVA } 3 \\
\text { ANOVA }\end{array}$ \\
\hline
\end{tabular}


Table 3 (cont.)

Independent and Dependent Variables for Research Questions 1, 2, 3, and 4

\begin{tabular}{|c|c|c|c|}
\hline Research Question & Independent Variable & $\begin{array}{c}\text { Dependent Variable } \\
(\mathrm{T}-\mathrm{Score})\end{array}$ & alysis Strategy \\
\hline & & Scale Score & \\
\hline RQ3a & $\begin{array}{l}\text { Assessment Time } \\
\text { (Pre vs. Post) }\end{array}$ & Attention Problems & $\begin{array}{l}\text { MANOVA } 4 \\
\text { ANOVA }\end{array}$ \\
\hline \multirow[t]{2}{*}{ RQ3b } & $\begin{array}{l}\text { Assessment Time } \\
\text { (Pre vs. Post) }\end{array}$ & Learning Problems & $\begin{array}{l}\text { MANOVA } 4 \\
\text { ANOVA }\end{array}$ \\
\hline & & Scale Score & \\
\hline RQ4a & $\begin{array}{l}\text { Assessment Time } \\
\text { (Pre vs. Post) }\end{array}$ & Adaptability & $\begin{array}{l}\text { MANOVA } 5 \\
\text { ANOVA }\end{array}$ \\
\hline RQ4b & $\begin{array}{l}\text { Assessment Time } \\
\text { (Pre vs. Post) }\end{array}$ & Social Skills & $\begin{array}{l}\text { MANOVA } 5 \\
\text { ANOVA }\end{array}$ \\
\hline RQ4c & $\begin{array}{l}\text { Assessment Time } \\
\text { (Pre vs. Post) }\end{array}$ & Leadership & $\begin{array}{l}\text { MANOVA } 5 \\
\text { ANOVA }\end{array}$ \\
\hline RQ4d & $\begin{array}{l}\text { Assessment Time } \\
\text { (Pre vs. Post) }\end{array}$ & Study Skills & $\begin{array}{l}\text { MANOVA } 5 \\
\text { ANOVA }\end{array}$ \\
\hline RQ4e & $\begin{array}{l}\text { Assessment Time } \\
\text { (Pre vs. Post) }\end{array}$ & Functional Communication & $\begin{array}{l}\text { MANOVA } 5 \\
\text { ANOVA }\end{array}$ \\
\hline
\end{tabular}

Note: Five MANOVAs were computed. To the extent that a given MANOVA yielded a significant finding $(p<.05)$, follow-up ANOVAs were computed. These follow-up ANOVAs determine if a particular dependent variable (e.g., Hyperactivity) increased or decreased significantly $(p<.05)$ from the Pre assessment (at time of referral for services) to the Post assessment (at time of one year from the referral for services). 


\section{Table 4}

Predictor Variables and Criterion Variables for Multiple Linear Regression Analyses (Research Questions 5, 6, 7, and 8)

\begin{tabular}{|c|c|}
\hline $\begin{array}{l}\text { Predictor Variables } \\
\text { Minutes of: }\end{array}$ & $\begin{array}{l}\text { Criterion Variables } \\
\text { Change in T-Score for: }\end{array}$ \\
\hline \multicolumn{2}{|l|}{$\begin{array}{c}\text { Research Question } 5 \text { (Externalizing Prol } \\
\text { Individual Counseling } \\
\text { Group Counseling } \\
\text { Family Education }\end{array}$} \\
\hline $\begin{array}{l}\text { Individual Counseling } \\
\text { Group Counseling } \\
\text { Family Education }\end{array}$ & Aggression \\
\hline $\begin{array}{l}\text { Individual Counseling } \\
\text { Group Counseling } \\
\text { Family Education }\end{array}$ & Conduct Problems \\
\hline $\begin{array}{c}\text { Research Question } 6 \text { (Internalizing Pro } \\
\text { Individual Counseling } \\
\text { Group Counseling } \\
\text { Family Education }\end{array}$ & Anxiety \\
\hline $\begin{array}{l}\text { Individual Counseling } \\
\text { Group Counseling } \\
\text { Family Education }\end{array}$ & Depression \\
\hline $\begin{array}{l}\text { Individual Counseling } \\
\text { Group Counseling } \\
\text { Family Education }\end{array}$ & Somatization \\
\hline $\begin{array}{l}\text { Research Question } 7 \text { (School Problems } \\
\text { Individual Counseling } \\
\text { Group Counseling } \\
\text { Family Education }\end{array}$ & Attention Problems \\
\hline $\begin{array}{l}\text { Individual Counseling } \\
\text { Group Counseling } \\
\text { Family Education }\end{array}$ & Learning Problems \\
\hline
\end{tabular}


Table 4 (cont.)

Predictor Variables and Criterion Variables for Multiple Linear Regression Analyses (Research Questions 5, 6, 7, and 8)

\begin{tabular}{|c|c|}
\hline $\begin{array}{l}\text { Predictor Variables } \\
\text { Minutes of: }\end{array}$ & $\begin{array}{l}\text { Criterion Variables } \\
\text { Change in T-Score for: }\end{array}$ \\
\hline $\begin{array}{c}\text { Research Question } 8 \text { (Adaptive Skills) } \\
\text { Individual Counseling } \\
\text { Group Counseling } \\
\text { Family Education }\end{array}$ & Adaptability \\
\hline $\begin{array}{l}\text { Individual Counseling } \\
\text { Group Counseling } \\
\text { Family Education }\end{array}$ & Social Skills \\
\hline $\begin{array}{l}\text { Individual Counseling } \\
\text { Group Counseling } \\
\text { Family Education }\end{array}$ & Leadership \\
\hline $\begin{array}{l}\text { Individual Counseling } \\
\text { Group Counseling } \\
\text { Family Education }\end{array}$ & Study Skills \\
\hline $\begin{array}{l}\text { Individual Counseling } \\
\text { Group Counseling } \\
\text { Family Education }\end{array}$ & Functional Communication \\
\hline
\end{tabular}


Table 5

Overall Multivariate Analysis of Variance (MANOVA) for Research Questions 1, 2, 3, and 4

\begin{tabular}{|c|c|c|c|c|}
\hline \multirow[t]{2}{*}{ Independent Variable } & \multirow[t]{2}{*}{ Dependent Variable } & \multicolumn{3}{|c|}{ MANOVA } \\
\hline & & $\mathrm{df}$ & $\mathrm{F}$ & $\mathrm{p}$ \\
\hline $\begin{array}{l}\text { Assessment Time } \\
\text { (Pre assessment vs } \\
\text { Post assessment) }\end{array}$ & $\begin{array}{l}\text { Externalizing Problems, } \\
\text { Internalizing Problems, } \\
\text { School Problems, and } \\
\text { Adaptive Skills }\end{array}$ & $(4,342)$ & 5.41 & $<.01 * *$ \\
\hline
\end{tabular}

Significance level: $p<.05$

$*$ indicates results are significant at $p<.05$

** indicates results are significant at $p<.01$ 
Table 6

One-way within subjects (repeated measures) Analysis of Variance for Research Questions 1, 2, 3 , and 4

\begin{tabular}{|c|c|c|c|c|}
\hline Independent Variable & Dependent Variable & $\mathrm{df}$ & $\begin{array}{l}\text { OVA } \\
\text { F }\end{array}$ & $\mathrm{p}$ \\
\hline $\begin{array}{l}\text { Assessment Time } \\
\text { (Pre assessment vs } \\
\text { Post assessment) }\end{array}$ & Externalizing Problems & $(1,345)$ & 20.35 & $<.01 * *$ \\
\hline $\begin{array}{l}\text { Assessment Time } \\
\text { (Pre assessment vs } \\
\text { Post assessment) }\end{array}$ & Internalizing Problems & $(1,345)$ & 9.45 & $<.01 * *$ \\
\hline $\begin{array}{l}\text { Assessment Time } \\
\text { (Pre assessment vs } \\
\text { Post assessment) }\end{array}$ & School Problems & $(1,345)$ & 6.69 & $<.01 * *$ \\
\hline $\begin{array}{l}\text { Assessment Time } \\
\text { (Pre assessment vs } \\
\text { Post assessment) }\end{array}$ & Adaptive Skills & $(1,345)$ & 3.33 & .07 \\
\hline
\end{tabular}

Significance level: $p<.05$

$*$ indicates results are significant at $p<.05$

** indicates results are significant at $p<.01$ 
Table 7

Overall Multivariate Analysis of Variance (MANOVA) for Research Questions 1a, 1b, and 1c

\begin{tabular}{lllll}
\hline Independent Variable & Dependent Variable & MANOVA & \\
& & df & F \\
\hline $\begin{array}{l}\text { Assessment Time } \\
\text { Pre assessment vs } \\
\text { Post assessment) }\end{array}$ & $\begin{array}{l}\text { Hyperactivity Problems, } \\
\text { Conduct Problems, and } \\
\text { Aggression Problems }\end{array}$ & & 7.08 & $<.01^{* *}$ \\
& $\begin{array}{l}(3,343) \\
\text { Aggrems }\end{array}$ & &
\end{tabular}

Significance level: $p<.05$

$*$ indicates results are significant at $p<.05$

$* *$ indicates results are significant at $p<.01$ 
Table 8

One-way within subjects (repeated measures) Analysis of Variance for Research Questions 1a, $1 b$, and $1 \mathrm{c}$

\begin{tabular}{|c|c|c|c|c|}
\hline Independent Variable & Dependent Variable & $\mathrm{df}$ & $\begin{array}{l}\text { OVA } \\
\text { F }\end{array}$ & $\mathrm{p}$ \\
\hline $\begin{array}{l}\text { Assessment Time } \\
\text { (Pre assessment vs } \\
\text { Post assessment) }\end{array}$ & Hyperactivity Problems & $(1,345)$ & 17.08 & $<.01 * *$ \\
\hline $\begin{array}{l}\text { Assessment Time } \\
\text { (Pre assessment vs } \\
\text { Post assessment) }\end{array}$ & Aggression Problems & $(1,345)$ & 18.77 & $<.01 * *$ \\
\hline $\begin{array}{l}\text { Assessment Time } \\
\text { (Pre assessment vs } \\
\text { Post assessment) }\end{array}$ & Conduct Problems & $(1,345)$ & 13.11 & $<.01 * *$ \\
\hline
\end{tabular}

Significance level: $p<.05$

$*$ indicates results are significant at $p<.05$

$* *$ indicates results are significant at $p<.01$ 
Table 9

Overall Multivariate Analysis of Variance (MANOVA) for Research Questions 2a, 2b, and 2c

\begin{tabular}{llll}
\hline Independent Variable & Dependent Variable & df & MANOVA \\
& & F & \\
\hline $\begin{array}{l}\text { Assessment Time } \\
\text { (Pre assessment vs } \\
\text { Post assessment) }\end{array}$ & $\begin{array}{l}\text { Anxiety Problems, } \\
\text { Depression Problems, and } \\
\text { Somatization Problems }\end{array}$ & & \\
\hline
\end{tabular}

Significance level: $p<.05$

$*$ indicates results are significant at $p<.05$

$* *$ indicates results are significant at $p<.01$ 
Table 10

One-way within subjects (repeated measures) Analysis of Variance for Research Questions 2a, $2 \mathrm{~b}$, and $2 \mathrm{c}$

\begin{tabular}{|c|c|c|c|c|}
\hline Independent Variable & Dependent Variable & $\mathrm{df}$ & $\begin{array}{l}\text { OVA } \\
\text { F }\end{array}$ & $\mathrm{p}$ \\
\hline $\begin{array}{l}\text { Assessment Time } \\
\text { (Pre assessment vs } \\
\text { Post assessment) }\end{array}$ & Anxiety Problems & $(1,345)$ & 4.55 & $<.01 * *$ \\
\hline $\begin{array}{l}\text { Assessment Time } \\
\text { (Pre assessment vs } \\
\text { Post assessment) }\end{array}$ & Depression Problems & $(1,345)$ & 9.94 & $<.01 * *$ \\
\hline $\begin{array}{l}\text { Assessment Time } \\
\text { (Pre assessment vs } \\
\text { Post assessment) }\end{array}$ & Somatization Problems & $(1,345)$ & 2.82 & .09 \\
\hline
\end{tabular}

Significance level: $p<.05$

$*$ indicates results are significant at $p<.05$

** indicates results are significant at $p<.01$ 
Table 11

Overall Multivariate Analysis of Variance (MANOVA) for Research Questions 3a and 3b

\begin{tabular}{llccc}
\hline Independent Variable & Dependent Variable & df & MANOVA & F \\
& & p & \\
\hline $\begin{array}{l}\text { Assessment Time } \\
\begin{array}{l}\text { Pre assessment vs } \\
\text { Post assessment) }\end{array}\end{array}$ & $\begin{array}{l}\text { Attention Problems and } \\
\text { Learning Problems }\end{array}$ & $(2,344)$ & 3.90 & $<.05^{*}$ \\
& & & &
\end{tabular}

Significance level: $p<.05$

$*$ indicates results are significant at $p<.05$

$* *$ indicates results are significant at $p<.01$ 


\section{Table 12}

One-way within subjects (repeated measures) Analysis of Variance for Research Questions 3a and $3 b$

Independent Variable

Dependent Variable

ANOVA

df $\mathrm{F} \quad \mathrm{p}$

Assessment Time

Attention Problems

$(1,345)$

7.77

$<.01 * *$

(Pre assessment vs

Post assessment)

Assessment Time

Learning Problems

$(1,345)$

2.76

.09

(Pre assessment vs

Post assessment)

Significance level: $p<.05$

$*$ indicates results are significant at $p<.05$

$* *$ indicates results are significant at $p<.01$ 


\section{Table 13}

Overall Multivariate Analysis of Variance (MANOVA) for Research Questions 4a, 4b, 4c, 4d, and $4 \mathrm{e}$

\begin{tabular}{|c|c|c|c|c|}
\hline Independent Variable & Dependent Variable & & $\mathrm{NOV}$ & \\
\hline & & $\mathrm{df}$ & $\mathrm{F}$ & $\mathrm{p}$ \\
\hline Assessment Time & Adaptability Deficits, & $(5,341)$ & 2.09 & .07 \\
\hline (Pre assessment vs & Social Skills Deficits, & & & \\
\hline Post assessment) & Leadership Deficits, & & & \\
\hline & Study Skills Deficits, and & & & \\
\hline & Functional Communication & & & \\
\hline & Deficits & & & \\
\hline
\end{tabular}

Significance level: $p<.05$

$*$ indicates results are significant at $p<.05$

$* *$ indicates results are significant at $p<.01$ 


\section{Table 14}

One-way within subjects (repeated measures) Analysis of Variance for Research Questions 4a, $4 \mathrm{~b}, 4 \mathrm{c}, 4 \mathrm{~d}$, and $4 \mathrm{e}$

\begin{tabular}{|c|c|c|c|c|}
\hline \multirow[t]{2}{*}{ Independent Variable } & \multirow[t]{2}{*}{ Dependent Variable } & \multicolumn{3}{|c|}{ ANOVA } \\
\hline & & $\mathrm{df}$ & $\mathrm{F}$ & $\mathrm{p}$ \\
\hline $\begin{array}{l}\text { Assessment Time } \\
\text { (Pre assessment vs } \\
\text { Post assessment) }\end{array}$ & Adaptability Deficits & $(1,345)$ & 8.07 & $<.01 * *$ \\
\hline $\begin{array}{l}\text { Assessment Time } \\
\text { (Pre assessment vs } \\
\text { Post assessment) }\end{array}$ & Social Skills Deficits & $(1,345)$ & 1.67 & .20 \\
\hline $\begin{array}{l}\text { Assessment Time } \\
\text { (Pre assessment vs } \\
\text { Post assessment) }\end{array}$ & Leadership Deficits & $(1,345)$ & .54 & .46 \\
\hline $\begin{array}{l}\text { Assessment Time } \\
\text { (Pre assessment vs } \\
\text { Post assessment) }\end{array}$ & Study Skills Deficits & $(1,345)$ & 3.49 & .06 \\
\hline $\begin{array}{l}\text { Assessment Time } \\
\text { (Pre assessment vs } \\
\text { Post assessment) }\end{array}$ & $\begin{array}{l}\text { Functional Communication } \\
\text { Deficits }\end{array}$ & $(1,345)$ & 2.97 & .09 \\
\hline
\end{tabular}

Significance level: $p<.05$

$*$ indicates results are significant at $p<.05$

$* *$ indicates results are significant at $p<.01$ 
Table 15

Research Question 5: 3 by 3 Chi-Square Matrix

Externalizing Problems

Category

Frequency Chi-Square
Observed (Expected)

Non-Significant to Non-Significant (0 to 0$)$

Non-Significant to At-Risk (0 to 1)

$157(124.6) \quad 75.80 \quad<.01 * *$

Non-Significant to Clinically Significant (0 to 2)

$29(43.4)$

$9 \quad(27.1)$

At-Risk to Non-Significant (1 to 0)

$40 \quad(51.7)$

At-Risk to At-Risk (1 to 1)

At-Risk to Clinically Significant (1 to 2)

$29(18.0)$

$12(11.2)$

Clinically Significant to Non-Significant (2 to 0)

Clinically Significant to At-Risk (2 to 1)

$24(44.7)$

Clinically Significant to Clinically Significant (2 to 2)

$19(15.6)$

$27(9.7)$

Total 346

Significance level: $p<.05$

$*$ indicates results are significant at $p<.05$

$* *$ indicates results are significant at $p<.01$

$(0=$ Non-Significant, $1=$ At-Risk, $2=$ Clinically Significant $)$ 
Table 16

Research Question 5a: 3 by 3 Chi-Square Matrix

Hyperactivity Problems

Category

Frequency Chi-Square
Observed (Expected)

Non-Significant to Non-Significant (0 to 0$)$

Non-Significant to At-Risk (0 to 1)

$155(120.9) \quad 67.58 \quad<.01 * *$

Non-Significant to Clinically Significant (0 to 2)

$28(44.7)$

$8 \quad(25.4)$

At-Risk to Non-Significant (1 to 0)

$42 \quad(55.1)$

At-Risk to At-Risk (1 to 1)

28 (20.4)

At-Risk to Clinically Significant (1 to 2)

17 (11.6)

Clinically Significant to Non-Significant (2 to 0)

$22(43.0)$

Clinically Significant to At-Risk (2 to 1)

25 (15.9)

Clinically Significant to Clinically Significant (2 to 2)

$21(9.0)$

Total

346

Significance level: $p<.05$

$*$ indicates results are significant at $p<.05$

$* *$ indicates results are significant at $p<.01$

$(0=$ Non-Significant, $1=$ At-Risk, $2=$ Clinically Significant $)$ 
Table 17

Research Question 5b: 3 by 3 Chi-Square Matrix

Aggression Problems

Category

Frequency Chi-Square
Observed (Expected)

Non-Significant to Non-Significant (0 to 0$)$

Non-Significant to At-Risk (0 to 1)

$166(134.4) \quad 88.30 \quad<.01 * *$

Non-Significant to Clinically Significant (0 to 2)

$26(33.6)$

$12(36.0)$

At-Risk to Non-Significant (1 to 0)

$45 \quad(50.7)$

At-Risk to At-Risk (1 to 1)

17 (12.7)

At-Risk to Clinically Significant (1 to 2)

15 (13.6)

Clinically Significant to Non-Significant (2 to 0)

$17(42.8)$

Clinically Significant to At-Risk (2 to 1)

$14(10.7)$

Clinically Significant to Clinically Significant (2 to 2)

34 (11.5)

Total 346

Significance level: $p<.05$

$*$ indicates results are significant at $p<.05$

$* *$ indicates results are significant at $p<.01$

$(0=$ Non-Significant, $1=$ At-Risk, $2=$ Clinically Significant $)$ 
Table 18

Research Question 5c: 3 by 3 Chi-Square Matrix

Conduct Problems

Category

Frequency Chi-Square
Observed (Expected)

Non-Significant to Non-Significant (0 to 0$)$

Non-Significant to At-Risk (0 to 1)

$178(151.4) \quad 58.07 \quad<.01 * *$

Non-Significant to Clinically Significant (0 to 2)

$26(41.4)$

$9 \quad(23.4)$

At-Risk to Non-Significant (1 to 0)

45 (52.6)

At-Risk to At-Risk (1 to 1)

19 (13.3)

At-Risk to Clinically Significant (1 to 2)

$10(8.1)$

Clinically Significant to Non-Significant (2 to 0)

$23(41.9)$

Clinically Significant to At-Risk (2 to 1)

$17(10.6)$

Clinically Significant to Clinically Significant (2 to 2)

$19(6.5)$

Total 346

Significance level: $p<.05$

$*$ indicates results are significant at $p<.05$

$* *$ indicates results are significant at $p<.01$

$(0=$ Non-Significant, $1=$ At-Risk, $2=$ Clinically Significant $)$ 
Table 19

Research Question 6: 3 by 3 Chi-Square Matrix

Internalizing Problems

Category

Frequency Chi-Square
Observed (Expected)

Non-Significant to Non-Significant (0 to 0$)$

Non-Significant to At-Risk (0 to 1)

$196(176.9) \quad 37.43 \quad<.01 * *$

Non-Significant to Clinically Significant (0 to 2)

$33 \quad(39.0)$

$12(25.1)$

At-Risk to Non-Significant (1 to 0)

36 (43.3)

At-Risk to At-Risk (1 to 1)

At-Risk to Clinically Significant (1 to 2)

$13(9.5)$

$10(6.1)$

Clinically Significant to Non-Significant (2 to 0)

Clinically Significant to At-Risk (2 to 1)

$22(33.8)$

$10 \quad(9.5)$

Clinically Significant to Clinically Significant (2 to 2)

14 (4.8)

Total

346

Significance level: $p<.05$

$*$ indicates results are significant at $p<.05$

$* *$ indicates results are significant at $p<.01$

$(0=$ Non-Significant, $1=$ At-Risk, $2=$ Clinically Significant $)$ 
Table 20

Research Question 6a: 3 by 3 Chi-Square Matrix

Anxiety Problems

Category

Frequency Chi-Square
Observed (Expected)

Non-Significant to Non-Significant (0 to 0$)$

Non-Significant to At-Risk (0 to 1)

Non-Significant to Clinically Significant (0 to 2)

$223(210.6) \quad 19.55<.01 * *$

$29(35.1)$

12 (18.3)

At-Risk to Non-Significant (1 to 0)

At-Risk to At-Risk (1 to 1)

$40 \quad(46.3)$

At-Risk to Clinically Significant (1 to 2)

$11(7.7)$

$7 \quad(4.0)$

Clinically Significant to Non-Significant (2 to 0)

$13(19.1)$

Clinically Significant to At-Risk (2 to 1)

$6 \quad(3.2)$

Clinically Significant to Clinically Significant (2 to 2)

$5 \quad(1.7)$

Total 346

Significance level: $p<.05$

$*$ indicates results are significant at $p<.05$

$* *$ indicates results are significant at $p<.01$

$(0=$ Non-Significant, $1=$ At-Risk, $2=$ Clinically Significant $)$ 
Table 21

Research Question 6b: 3 by 3 Chi-Square Matrix

Depression Problems

Category

Frequency Chi-Square
Observed (Expected)

Non-Significant to Non-Significant (0 to 0$)$

Non-Significant to At-Risk (0 to 1)

$175(155.0) \quad 30.63 \quad<.01 * *$

Non-Significant to Clinically Significant (0 to 2)

$28 \quad(39.7)$

15 (23.3)

At-Risk to Non-Significant (1 to 0)

$50 \quad(58.3)$

At-Risk to At-Risk (1 to 1)

22 (14.9)

At-Risk to Clinically Significant (1 to 2)

$10(8.8)$

Clinically Significant to Non-Significant (2 to 0)

Clinically Significant to At-Risk (2 to 1)

$21(32.7)$

Clinically Significant to Clinically Significant (2 to 2)

$13(8.4)$

$12(4.9)$

Total 346

Significance level: $p<.05$

$*$ indicates results are significant at $p<.05$

$* *$ indicates results are significant at $p<.01$

$(0=$ Non-Significant, $1=$ At-Risk, $2=$ Clinically Significant $)$ 
Table 22

Research Question 6c: 3 by 3 Chi-Square Matrix

Somatization Problems

Category

Frequency Chi-Square
Observed (Expected)

Non-Significant to Non-Significant (0 to 0$)$

Non-Significant to At-Risk (0 to 1)

$225(210.7) \quad 48.85 \quad<.01 * *$

Non-Significant to Clinically Significant (0 to 2)

38 (44.8)

4 (11.6)

At-Risk to Non-Significant (1 to 0)

37 (39.5)

At-Risk to At-Risk (1 to 1)

At-Risk to Clinically Significant (1 to 2)

$9 \quad(8.4)$

$4 \quad(11.6)$

Clinically Significant to Non-Significant (2 to 0)

Clinically Significant to At-Risk (2 to 1)

$11(22.9)$

11 (4.9)

Clinically Significant to Clinically Significant (2 to 2)

$7 \quad(1.3)$

Total 346

Significance level: $p<.05$

$*$ indicates results are significant at $p<.05$

$* *$ indicates results are significant at $p<.01$

$(0=$ Non-Significant, $1=$ At-Risk, $2=$ Clinically Significant $)$ 
Table 23

Research Question 7: 3 by 3 Chi-Square Matrix

\begin{tabular}{|c|c|c|c|}
\hline $\begin{array}{l}\text { School Problems } \\
\text { Category }\end{array}$ & Frequency & Chi-Square & $\mathrm{p}$ \\
\hline Non-Significant to Non-Significant (0 to 0 ) & $158(131.1)$ & 80.60 & $<.01 * *$ \\
\hline Non-Significant to At-Risk ( 0 to 1$)$ & $46(63.1)$ & & \\
\hline Non-Significant to Clinically Significant (0 to 2 ) & $6 \quad(15.8)$ & & \\
\hline At-Risk to Non-Significant ( 1 to 0 ) & $49 \quad(61.8)$ & & \\
\hline At-Risk to At-Risk ( 1 to 1 ) & $44 \quad(29.8)$ & & \\
\hline At-Risk to Clinically Significant (1 to 2) & $6 \quad(7.4)$ & & \\
\hline Clinically Significant to Non-Significant ( 2 to 0 ) & $9 \quad(23.1)$ & & \\
\hline Clinically Significant to At-Risk (2 to 1) & $14(11.1)$ & & \\
\hline Clinically Significant to Clinically Significant (2 to 2) & $14(2.8)$ & & \\
\hline Total & 346 & & \\
\hline
\end{tabular}

Significance level: $p<.05$

$*$ indicates results are significant at $p<.05$

$* *$ indicates results are significant at $p<.01$

$(0=$ Non-Significant, $1=$ At-Risk, $2=$ Clinically Significant $)$ 
Table 24

Research Question 7a: 3 by 3 Chi-Square Matrix

Attention Problems

Category

Frequency Chi-Square
Observed (Expected)

Non-Significant to Non-Significant (0 to 0$)$

Non-Significant to At-Risk (0 to 1)

Non-Significant to Clinically Significant (0 to 2)

$123(103.9) \quad 26.06 \quad<.01 * *$

$53(65.8)$

$2(8.2)$

At-Risk to Non-Significant (1 to 0)

73 (86.4)

At-Risk to At-Risk (1 to 1)

$64(54.8)$

At-Risk to Clinically Significant (1 to 2)

$11(6.8)$

Clinically Significant to Non-Significant (2 to 0)

Clinically Significant to At-Risk (2 to 1)

$6 \quad(11.7)$

Clinically Significant to Clinically Significant (2 to 2)

$11(7.4)$

$3(0.9)$

Total

346

Significance level: $p<.05$

$*$ indicates results are significant at $p<.05$

$* *$ indicates results are significant at $p<.01$

$(0=$ Non-Significant, $1=$ At-Risk, $2=$ Clinically Significant $)$ 
Table 25

Research Question 7b: 3 by 3 Chi-Square Matrix

Learning Problems

Category

Frequency Chi-Square
Observed (Expected)

Non-Significant to Non-Significant (0 to 0$)$

Non-Significant to At-Risk (0 to 1)

$186(158.2) \quad 82.15 \quad<.01 * *$

Non-Significant to Clinically Significant (0 to 2)

$38 \quad(53.6)$

$8 \quad(20.1)$

At-Risk to Non-Significant (1 to 0 )

$41 \quad(55.2)$

At-Risk to At-Risk (1 to 1)

At-Risk to Clinically Significant (1 to 2)

$32(18.7)$

$8 \quad(7.0)$

Clinically Significant to Non-Significant (2 to 0)

Clinically Significant to At-Risk (2 to 1)

$9 \quad(22.5)$

$10(7.6)$

Clinically Significant to Clinically Significant (2 to 2)

14 (2.9)

Total

346

Significance level: $p<.05$

$*$ indicates results are significant at $p<.05$

$* *$ indicates results are significant at $p<.01$

$(0=$ Non-Significant, $1=$ At-Risk, $2=$ Clinically Significant $)$ 
Table 26

Research Question 8: 3 by 3 Chi-Square Matrix

Adaptive Skills Deficits

Category

Frequency Chi-Square
Observed (Expected)

Non-Significant to Non-Significant (0 to 0$)$

Non-Significant to At-Risk (0 to 1)

Non-Significant to Clinically Significant (0 to 2)

$139(113.1) \quad 32.85 \quad<.01 * *$

$47(70.3)$

$4 \quad(6.6)$

At-Risk to Non-Significant (1 to 0)

$60 \quad(81.6)$

At-Risk to At-Risk (1 to 1)

At-Risk to Clinically Significant (1 to 2)

$70 \quad(50.7)$

$7 \quad(4.8)$

Clinically Significant to Non-Significant (2 to 0)

Clinically Significant to At-Risk (2 to 1)

$7 \quad(11.3)$

Clinically Significant to Clinically Significant (2 to 2)

$11(7.0)$

$1 \quad(0.7)$

Total 346

Significance level: $p<.05$

$*$ indicates results are significant at $p<.05$

$* *$ indicates results are significant at $p<.01$

$(0=$ Non-Significant, $1=$ At-Risk, $2=$ Clinically Significant $)$ 
Table 27

Research Question 8a: 3 by 3 Chi-Square Matrix

Adaptability Deficits

Category

Frequency Chi-Square
Observed (Expected)

Non-Significant to Non-Significant (0 to 0$)$

Non-Significant to At-Risk (0 to 1)

Non-Significant to Clinically Significant (0 to 2)

$150(122.9) \quad 37.81 \quad<.01 * *$

$40 \quad(61.7)$

$6 \quad(11.3)$

At-Risk to Non-Significant (1 to 0)

53 (73.4)

At-Risk to At-Risk (1 to 1)

$54(36.9)$

At-Risk to Clinically Significant (1 to 2)

$10(6.8)$

Clinically Significant to Non-Significant (2 to 0)

Clinically Significant to At-Risk (2 to 1)

$14(20.7)$

Clinically Significant to Clinically Significant (2 to 2)

$15(10.4)$

4 (1.9)

Total 346

Significance level: $p<.05$

$*$ indicates results are significant at $p<.05$

$* *$ indicates results are significant at $p<.01$

$(0=$ Non-Significant, $1=$ At-Risk, $2=$ Clinically Significant $)$ 
Table 28

Research Question 8b: 3 by 3 Chi-Square Matrix

Social Skills Deficits

Category

Frequency Chi-Square
Observed (Expected)

Non-Significant to Non-Significant (0 to 0 )

Non-Significant to At-Risk (0 to 1)

$149(129.2) \quad 25.67 \quad<.01 * *$

Non-Significant to Clinically Significant (0 to 2)

54 (73.0)

4 (4.8)

At-Risk to Non-Significant (1 to 0 )

$59 \quad(71.2)$

At-Risk to At-Risk (1 to 1)

$51(40.2)$

At-Risk to Clinically Significant (1 to 2)

4 (2.6)

Clinically Significant to Non-Significant (2 to 0)

Clinically Significant to At-Risk (2 to 1)

$8 \quad(15.6)$

$17 \quad(8.8)$

Clinically Significant to Clinically Significant (2 to 2)

$0 \quad(0.6)$

Total 346

Significance level: $p<.05$

$*$ indicates results are significant at $p<.05$

$* *$ indicates results are significant at $p<.01$

$(0=$ Non-Significant, $1=$ At-Risk, $2=$ Clinically Significant $)$ 
Table 29

Research Question 8c: 3 by 3 Chi-Square Matrix

Leadership Deficits

Category

Frequency Chi-Square
Observed (Expected)

Non-Significant to Non-Significant (0 to 0$)$

Non-Significant to At-Risk (0 to 1)

Non-Significant to Clinically Significant (0 to 2)

$195(174.9) \quad 27.73 \quad<.01 * *$

48 (67.7)

$1 \quad(1.4)$

At-Risk to Non-Significant (1 to 0)

At-Risk to At-Risk (1 to 1)

At-Risk to Clinically Significant (1 to 2)

52 (71.2)

$47(27.7)$

$1 \quad(0.6)$

Clinically Significant to Non-Significant (2 to 0)

Clinically Significant to At-Risk (2 to 1)

$1 \quad(1.4)$

Clinically Significant to Clinically Significant (2 to 2)

$1 \quad(0.6)$

$0 \quad(0.0)$

Total 346

Significance level: $p<.05$

$*$ indicates results are significant at $p<.05$

$* *$ indicates results are significant at $p<.01$

$(0=$ Non-Significant, $1=$ At-Risk, $2=$ Clinically Significant $)$ 
Table 30

Research Question 8d: 3 by 3 Chi-Square Matrix

Study Skills Deficits

Category

Frequency Chi-Square
Observed (Expected)

Non-Significant to Non-Significant (0 to 0$)$

Non-Significant to At-Risk (0 to 1)

$142(114.3) \quad 49.24 \quad<.01 * *$

Non-Significant to Clinically Significant (0 to 2)

$46 \quad(66.2)$

$3 \quad(10.5)$

At-Risk to Non-Significant (1 to 0)

$62 \quad(82.0)$

At-Risk to At-Risk (1 to 1)

At-Risk to Clinically Significant (1 to 2)

$63 \quad(47.5)$

$12(7.5)$

Clinically Significant to Non-Significant (2 to 0)

Clinically Significant to At-Risk (2 to 1)

3 (10.8)

$11(6.2)$

Clinically Significant to Clinically Significant (2 to 2)

$4 \quad(1.0)$

Total 346

Significance level: $p<.05$

$*$ indicates results are significant at $p<.05$

$* *$ indicates results are significant at $p<.01$

$(0=$ Non-Significant, $1=$ At-Risk, $2=$ Clinically Significant $)$ 
Table 31

Research Question 8e: 3 by 3 Chi-Square Matrix

Functional Communication Deficits

Category

Frequency Chi-Square
Observed (Expected)

Non-Significant to Non-Significant (0 to 0 )

Non-Significant to At-Risk (0 to 1)

Non-Significant to Clinically Significant (0 to 2)

$167(142.7) \quad 42.54 \quad<.01 * *$

$38 \quad(55.5)$

$6 \quad(12.8)$

At-Risk to Non-Significant (1 to 0)

$58 \quad(73.0)$

At-Risk to At-Risk (1 to 1)

$41(28.4)$

At-Risk to Clinically Significant (1 to 2)

$9 \quad(6.6)$

Clinically Significant to Non-Significant (2 to 0)

Clinically Significant to At-Risk (2 to 1)

$9 \quad(18.3)$

$12(7.1)$

Clinically Significant to Clinically Significant (2 to 2)

$6 \quad(1.6)$

Total 346

Significance level: $p<.05$

$*$ indicates results are significant at $p<.05$

$* *$ indicates results are significant at $p<.01$

$(0=$ Non-Significant, $1=$ At-Risk, $2=$ Clinically Significant $)$ 